The copyright of this thesis vests in the author. No quotation from it or information derived from it is to be published without full acknowledgement of the source. The thesis is to be used for private study or noncommercial research purposes only.

Published by the University of Cape Town (UCT) in terms of the non-exclusive license granted to UCT by the author. 


\title{
HYBRID SYNTHESIS METHOD FOR MASS EXCHANGE NETWORKS
}

\author{
ANDREW KHUTSO MSIZA
}

A Thesis presented for the degree of Master of Science in Engineering In Chemical Engineering

Supervisor: Associate Professor D.M. Fraser

December 2001 


\section{Synopsis}

Process synthesis can be approached from three techniques: heuristics, physical and thermodynamic insight, and mathematical programming. Hybrid methods where two or all of the synthesis methods are combined are now becoming used, taking advantage of the combined strength of the individual techniques. In this thesis the option of combining pinch analysis (a physical and thermodynamic insight-based approach) and superstructure-based programming is explored. The synthesis philosophy is based on the observation that pinch targets provide, ahead of detailed design, a measure of how best a flowsheet can meet design requirements and that mathematical programming solutions, in the absence of a knowledge of a global optimum, cannot be regarded as global. The combination is therefore that the solutions obtained from the MINLP method are improved once there are targets available. This observation alone merely exposes the quality of the MINLP solution but more importantly within the hybrid method, is a requirement for the involvement of the designer to modify the MINLP model and evaluate the solutions it generates.

Embedded within the process of developing flowsheet targets is the use of driving force diagrams and sizing techniques that are graphically-based such the McCabe-Thiele construction for the determination of the number of stages. The translation of these tools towards the optimisation-based methods requires the implementation of approximation methods and the development of logical operators. The approximation methods developed in this thesis are aimed to provide an analytical function for the determination of the number of stages in a mass exchanger when the equilibrium function for the absorption is non-linear and the assumptions for the use of the Kremser equation are no longer valid. The number of stages are determined to be the ratio of the change in a composition of a stream and the average driving force between the contacting streams. Logical operators are implemented to handle the choice of a Kremser equation subject to the value of the absorption factor. While it may be argued that the choice of appropriate programming environments should eliminate the need for the ad-hoc development of logical operators, 
currently, the selected environment (GAMS) has already implemented in it, commercially available solvers which overcome the need to be concerned with the details of the solution algorithm itself.

A çase study is solved using the hybrid method and integrating within the synthesis process, an experimental campaign for the determination of thermodynamic data. The iterative process of integrating an experimental program within the tasks of process synthesis allows for additional trade-offs to be included in the design process. Traditional synthesis methods for mass exchange networks assume that the plant to be synthesised is isothermal and isobaric and hence the equilibrium relation for the absorption process is uniform throughout the plant. Where this assumption is relaxed, the equilibrium function will have to be developed for every temperature and pressure that the exchangers might be expected to operate at. Hence additional experimentation is needed in order to generate and use an appropriate equilibrium function and if need be, an alternative mass separating agent.

Concerning the generation of equilibrium functions, a Deshmuk-Mather model (as opposed to the Kent and Eisenberg model) was used. The Deshmuk-Mather model takes into account the interaction, fugacity and activity coefficients of the reacting species.

In the industrial case study, there were two processing facilities considered, with each facility using a different MSA (DEA or MEA) to remove $\mathrm{H}_{2} \mathrm{~S}$ from rich gas streams. The different cases that were studied included the option of optimising the DEA and MEA facilities separately, using the DEA in the MEA system or vice-versa and simultaneously optimising both facilities. The hybrid model illustrated that the option of integrating the two facilities leads to a major saving in operating costs subject to a low capital investmentrequirement. 


\section{Declaration}

The work in this thesis is based on research carried out at the Process Systems Engineering Research Group, Department of Chemical Engineering, University of Cape Town, South Africa in collaboration with the Technical University of Budapest, Hungary. No part of this thesis has been submitted in support of an application for another degree or qualification of this or any other institution of learning.

Andrew Khutso Msiza 


\title{
Acknowledgements
}

\author{
Motho ke motho ka batho \\ African proverb
}

This work could not have been completed without a number of people I would like to acknowledge here.

I would like to extend my sincerest gratitude to Associate Professor Duncan Fraser for his supervision and especially for giving me the freedom in the way in which I proceeded with the research topic. The high standard he demands of his students was a challenge that I have come to enjoy and definitely a characteristic that I aspire to.

Thanks to Professor Zsolt Fonyo and Associate Professor Edo Rev for the many valuable discussions and collaboration we had over the duration of this research. My visits to the Technical University of Budapest brought many new directions to this research and the friends I made were wonderful.

I would also like to thank Professor Mahmoud El-Halwagi of Auburn University for his willingness to answer my questions and Professor Alan Mather of Alberta University for making available the Deshmuk-Mather model for the generation of VLE data.

Many important contributions were made by Zsolt Szitkai who gave this work the start it so much needed through his advice and knowledge of MINLP.

Mathew Marx for making available the details of the hybrid retrofit case study.

I also owe much gratitude to Ben Knights who taught me everything I know about UNIX and who, on many occasions, saved me from many a computing disaster. Never had so many cups of coffee boet!

This project would not have been successful without the financial support from SASOL and the National Research Foundation. 
The students and staff in the Chemical Engineering Department and colleagues at the Student Housing and Accommodation Office who made my stay in Cape Town a memorable one.

To all the members of G.S.E.P.K.O.P.P, without your support, love and prayers my accomplishments would still be but dreams. 
To the city that brought new life to my world

To the mountain at whose foot I found long lasting wisdom

To the shores that washed my many struggles away

But most of all,

To the little angel from whom nations of the world will find their answers 


\section{Contents}

Synopsis

1 Introduction 1

1.1 Process Synthesis . . . . . . . . . . . . . . . . . 1

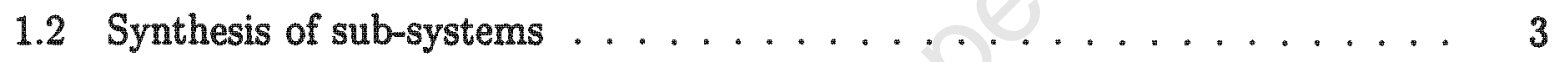

1.3 Pinch Analysis for MENS ...................... 4

1.4 Mixed integer non-linear programming . . . . . . . . . . . . . 4

1.5 Motivation and Goals ................... 5

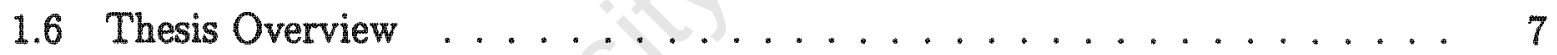

2 Literature Review 1

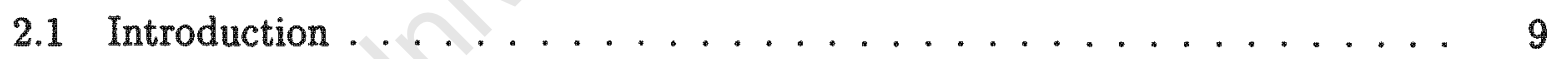

2.2 Pinch Technology $\ldots \ldots \ldots \ldots \ldots$

2.2.1 Operating cost targets ................... 14

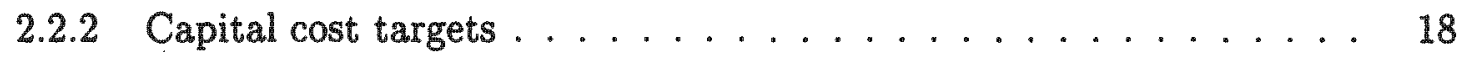

2.2 .3 Supertargeting ............................ 22

2.2 .4 Design from supertargets ..................... 23

2.2 .5 Pinch analysis summary ................... 26

2.3 Mathematical programming ..................... 27

2.3.1 Linear programming .................... 27 
2.3.2 Mixed integer linear programming . . . . . . . . . . . . 29

2.3.3 Non-linear programming . . . . . . . . . . . . . 30

2.3.4 Mixed-integer non-linear programming ............ 31

2.3.5 Computational issues ..................... 37

2.3 .6 Summary ........................... 41

2.4 Hybrid synthesis-tools . . . . . . . . . . . . . . 43

2.4.1 Waste Interception Networks .............. 44

2.4.2 Integrated design of waste water networks . . . . . . . . . 44

2.4.3 Insight-based superstructure . . . . . . . . . . . 46

2.4.4 Hybrid tools: Concluding remarks . . . . . . . . . . . . 47

2.5 Thesis Contributions .......................... 48

3 Sizing models $\quad 50$

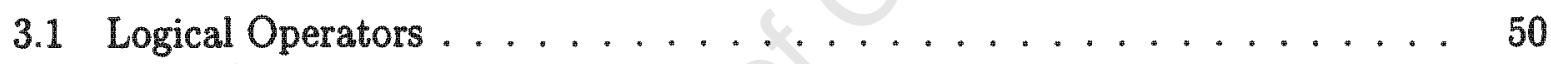

3.2 Approximating ideal stages . . . . . . . . . . . . . . 56

3.2 .1 Approximation philosophy ............... 56

3.2 .2 Approximation accuracy ...................... 58

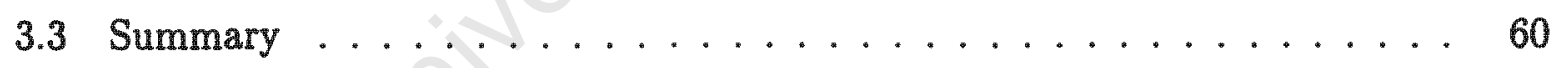

4 Hybrid Synthesis Method $\quad 62$

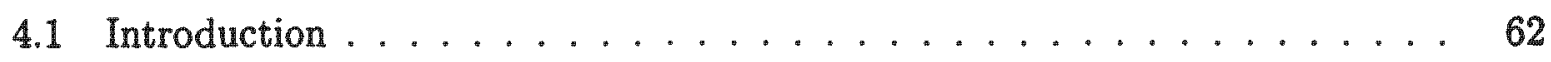

4.1.1 The Hybrid synthesis philosophy ............... 62

4.1.2 Problem statement ...................... 64

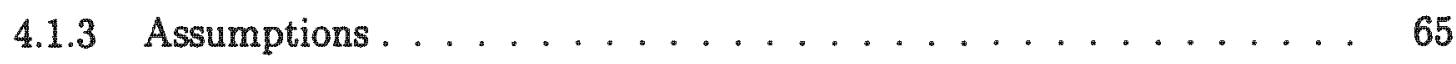

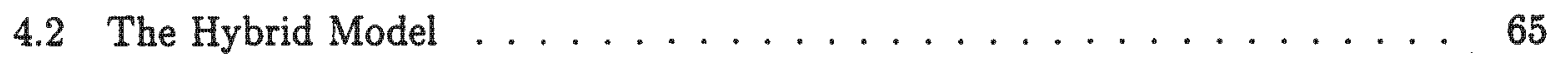

4.2.1 The size of the subnetworks ............... 66

4.2 .2 Defining stream bypass . . . . . . . . . . . . . 66 
4.2 .3 Feasibility constraints . . . . . . . . . . . . 68

4.2 .4 Sizing models . . . . . . . . . . . . . . . . . . 69

4.2 .5 Minimising non-linearities $\ldots \ldots \ldots \ldots \ldots \ldots$

4.2 .6 Logical constraints . . . . . . . . . . . . . 70

4.2 .7 Initialisation strategy $\ldots \ldots \ldots \ldots \ldots \ldots \ldots$

4.2 .8 Model solver . . . . . . . . . . . . . . . . 7

4.3 Case Studies . . . . . . . . . . . . . . . . . . . . . 72

$4.3 .1 \mathrm{SO}_{2}$ removal $\ldots \ldots \ldots \ldots \ldots \ldots \ldots \ldots \ldots \ldots \ldots$

4.3.2 Dephenolisation . . . . . . . . . . . . . 75

4.3 .3 Regeneration . . . . . . . . . . . . . 81

4.3.4 Reactive mass exchange networks $\ldots \ldots \ldots \ldots . \ldots 83$

4.4 Pinch-structure independent initialisations ... . . . . . . . 88

Remarks . . . . . . . . . . . . . . 96

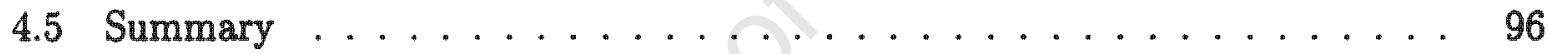

5 Extension to an industrial problem 98

5.1 Review . . . . . . . . . . . . . . . . . . . . . 99

5.1 .1 Topology constrained design $\ldots \ldots \ldots \ldots \ldots . \ldots 99$

5.1 .2 Solubility data prediction $\ldots \ldots \ldots \ldots \ldots$

5.2 The case study . . . . . . . . . . . . . . . . . . 104

5.2 .1 Problem description $\ldots \ldots \ldots \ldots \ldots$. . . . . . 104

5.2 .2 Equilibrium function generation $\ldots \ldots \ldots \ldots \ldots$

5.3 Analysis of various MEN options . . . . . . . . . . . 107

5.3.1 Maintaining the current facility layout . . . . . . . 110

5.3 .2 Interchanging the MSAs $\ldots \ldots \ldots \ldots \ldots \ldots \ldots$

5.3 .3 Total Integration $\ldots \ldots \ldots \ldots \ldots \ldots \ldots \ldots$

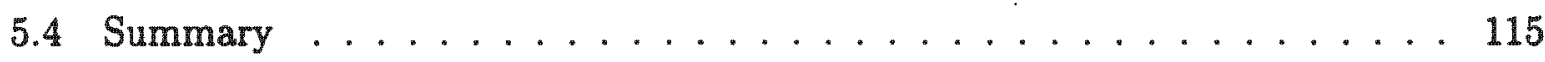


6 Conclusions $\quad 118$

$\begin{array}{ll}\text { References } & 123\end{array}$

$\begin{array}{lr}\text { Appendix } & 124\end{array}$

$\begin{array}{lr}\text { A Stream Data } & 124\end{array}$

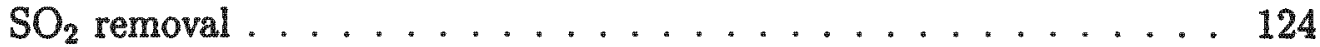

$\begin{array}{lr}\text { B Basic sizing expressions } & 129\end{array}$

Exchanger Height . . . . . . . . . . . . . . . 129

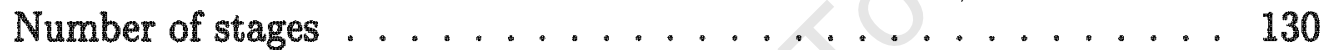

Mass of continuous contactor $M_{p s m, i j s} \ldots \ldots 130$

Mass of stagewise contactor $M_{p s m, i j s} \ldots \ldots \ldots 131$

Exchanger Volume $V_{i j s} \ldots \ldots \ldots 132$

Number of stages for non-linear equilibrium functions . . . . . 133

C MINLP Model Equations

D Case Study Additional Information $\quad 139$

E Additional Data on the MTA method 144 


\section{List of Figures}

1.1 Hierarchal levels of the synthesis process ................ 2

2.1 A mass exchange network .......................... 12

2.2 Construction of the rich composite curve .............. 15

2.3 Construction of the lean composite curve using $y^{*}$ compositions. . . . . 16

2.4 Combined composite curves to locate the pinch point and determine MSA

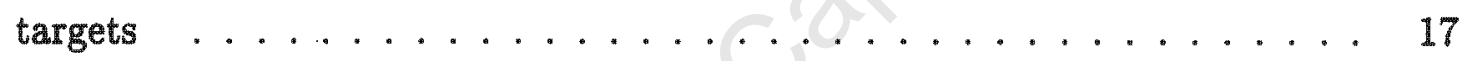

2.5 Construction of the $y-y^{*}$ curve for sizing exchangers . . . . . . . . 19

2.6 Exchanger sizing parameters from a $y-y^{*}$ curve plot . . . . . . . . . 22

2.7 Supertarget diagram for the selection of an optimum minimum composition

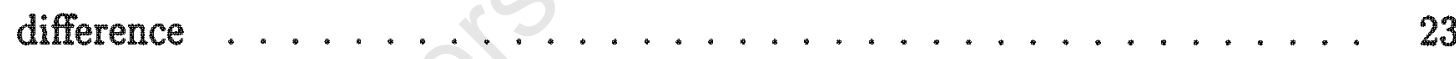

2.8 Grid diagram for capital cost targeting and network generation . . . . . . 24

2.9 Mass balance around the $k^{\text {th }}$ composition interval .......... 28

$2.10 \mathrm{I}-\mathrm{S}-\mathrm{O}$ representation of a dynamical system $\ldots \ldots \ldots . \ldots . \ldots . \ldots$

2.11 Alternative $[-$ S-O representation of a dynamic system . . . . . . . 33

2.12 The hyperstructure for the rich and lean streams $\ldots \ldots . \ldots 35$

2.13 A typical interval in a reducible superstructure . . . . . . . . . 46

3.1 The logical operator based on the binary variable approximation . . . . . 54

3.2 The use of the McCabe-Thiele method for non-linear equilibria. . . . . . 57

3.3 Comparison of the number of stages from the Kremser equation and from the MTA method ...................... 59 
4.1 The philosophy of the hybrid synthesis method $\ldots \ldots \ldots 63$

4.2 Definition of stream-bypass and stream-recycle in the MINLP model . . . 67

4.3 Pinch solution for the $\mathrm{SO}_{2}$ removal problem. Capital cost $=\$ 860000 \ldots 73$

4.4 Hybrid solution for the $\mathrm{SO}_{2}$ removal problem. Capital cost $=\$ 321000 \ldots 74$

4.5 A pinch (Capital cost $=\$ 448000$ ) (a) and a hybrid-based (Capital cost $=$ $\$ 488000$ ) (b) solution for $\mathrm{SO}_{2}$ removal problem when considering packed columns ......................... 76

4.6 Grid diagram for dephenolisation example $\ldots \ldots \ldots \ldots 77$

4.7 Optimum pinch solution for the dephenolisation example. Capital cost $=\$ 158400 \mathrm{k}$, Operating cost $=\$ 67000 \ldots \ldots \ldots \ldots$

4.8 Optimum hybrid solution for the dephenolisation example. Capital cost $=$ $\$ 235000$, Operating cost $=\$ 158400 \ldots \ldots \ldots \ldots$

4.9 Hybrid solution for the mixed equipment-type example. Capital cost $=$ $\$ 119000$, Operating cost $=\$ 433000 \ldots \ldots \ldots \ldots \ldots$

4.10 Pinch based regeneration network. Capital cost $=\$ 93500$, Operating cost $=$ $\$ 598500 \ldots \ldots \ldots \ldots \ldots \ldots \ldots . \ldots \ldots$

4.11 Hybrid regeneration example. Capital cost $=\$ 98300$, Operating cost $=$ $\$ 621700 \ldots \ldots \ldots \ldots \ldots \ldots \ldots$

4.12 The pinch solution for the $\mathrm{H}_{2} \mathrm{~S}$ removal case. Capital cost $=\$ 28000$, No operating cost

4.13 Hybrid solution for the $\mathrm{H}_{2} \mathrm{~S}$ removal problem. Capital cost $=\$ 32000$, No

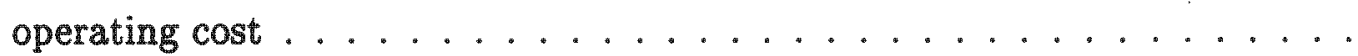

4.14 Network design based on pinch analysis. Capital cost $=\$ 535000$, No operating cost

4.15 Proposed reactive mass exchange network with the sizing based on the developed approximation method. Capital cost $=\$ 512000$, No operating cost

4.16 Driving force diagram for the dephenolisation example $\ldots \ldots \ldots \ldots$

4.17 Proposed initial structure independent of the pinch structure. Capital cost $=\$ 6000$, Operating cost $=\$ 2230000$ 
4.18 Driving force plot for the initialisation with external MSA only . . . . 90

4.19 Proposed network from analysis of driving force plot to reduce outlet composition of Ex6. Capital cost $=18400 \$$, Operating cost $=\$ 942300 \ldots \ldots 92$

4.20 Driving force plot for the initialisation with shared loads for Ex6 . . . . 92

4.21 The initialisation (a) and solution (b) to the parallel-series initialisation. Capital cost $=\$ 80000$, Operating cost $=\$ 192000 \ldots \ldots \ldots \ldots$

4.22 Driving force usage for the parallel-series initialisation . . . . . . . . .

5.1 Solubility data in literature $\ldots \ldots \ldots \ldots 10 \ldots \ldots$

5.2 Refinery flowsheet for the $\mathrm{H}_{2} \mathrm{~S}-\mathrm{MEA}$ section $(\mathrm{N10}) \ldots \ldots \ldots \ldots$

5.3 Refinery flowsheet for the $\mathrm{H}_{2} \mathrm{~S}-\mathrm{DEA}$ section $(\mathrm{N} 20) \ldots \ldots \ldots \ldots$

5.4 Options for the use of MSA in the $\mathrm{H}_{2} \mathrm{~S}$ removal case study $\ldots \ldots \ldots 106$

5.5 Effect of $\mathrm{CO}_{2}$ on the solubility of $\mathrm{H}_{2} \mathrm{~S}$ in MEA . . . . . . 108

5.6 Savings and investment distribution for selected design options . . . . . 109

5.7 New MEA flowsheet $(\mathrm{N} 1) \ldots \ldots \ldots \ldots \ldots \ldots$

5.8 New DEA flowsheet $(\mathrm{N} 2) \ldots \ldots \ldots \ldots \ldots \ldots \ldots \ldots$

5.9 Networks illustrating (a) DEA replacing MEA (N3) and (b) MEA replacing

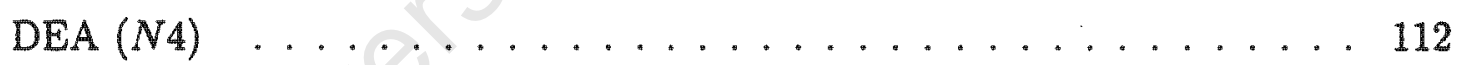

5.10 Totally integrated $\mathrm{H}_{2} \mathrm{~S}$ removal facility employing only MEA (N5) . . 114

5.11 Totally integrated $\mathrm{H}_{2} \mathrm{~S}$ removal facility employing both MEA and DEA (N5

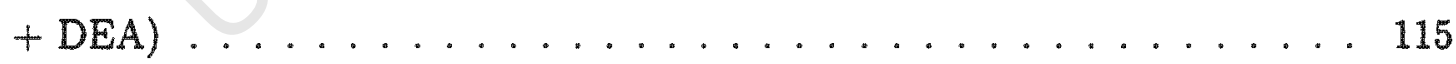

5.12 Analysis of the resulting circulation rates from various design options $\ldots 116$ 


\section{List of Tables}

2.1 The comparison of HEN and MEN technology . . . . . . . . . 12

2.2 Existing targets in the HEN and MEN technologies ........... 13

3.1 Comparison of the logical operators to use with the Kremser equation . . 56

3.2 Number of stages determined by different methods . . . . . . . . . . . 60

4.1 Exchanger classification table for the mixed equipment-type example . . 79

4.2 Minimum allowable composition differences for the streams in the $\mathrm{H}_{2} \mathrm{~S}$ removal 84

4.3 Number of equilibrium stages obtained for the reactive mass exchange network. 88

4.4 Costs of the optimum solutions when the solver is initialised from structures that are not pinch-based . . . . . . . . . . . . . 95

5.1 Equilibrium functions for the MEA system . . . . . . . . . . . 107

5.2 Summary of results for the gas sweetening case study. . . . . . . . 108

5.3 Prices for bulk sales of alkanoamines(?) . . . . . . . . . . 113

A.1 Stream data for $\mathrm{SO}_{2}$ removal: Base case . . . . . . . . . . . 124

A.2 Cost data for $\mathrm{SO} 2$ removal: Base case . . . . . . . . . . . . . . 124

A.3 Stream data for $\mathrm{SO}_{2}$ removal: Scenario2 $\ldots \ldots \ldots \ldots$

A.4 Equipment data for $\mathrm{SO}_{2}$ removal: Scenario2 $\ldots \ldots \ldots 125$

A.5 Stream data for dephenolisation: base case . . . . . . . . . 125

A.6 Equipment data for dephenolisation: base case . . . . . . . . . . 126 
A.7 Stream data for dephenolisation: mixed-equipment $\ldots \ldots \ldots \ldots$

A.8 Equipment data for dephenolisation: mixed-equipment $\ldots \ldots \ldots \ldots$

A.9 Stream data for regeneration example . . . . . . . . . . . 127

A.10 Equipment data for regeneration case . . . . . . . . . . . . 127

A.11 Stream data for REAMEN example with DEA . . . . . . . . . 127

A.12 Stream data for RAEMEN example with MEA . . . . . . . . . . . 128

A.13 Equipment data for REAMEN example with MEA . . . . . . . . . 128

D.1 Stream data case study . . . . . . . . . . . . . . . . . . 139

D.2 Equipment data for gas sweetening case study . . . . . . . . . 140

D.3 Partial Pressure and Loading data for the H2S-MEA system at $38^{\circ} \mathrm{C} . \quad \ldots 140$

D.4 Partial Pressure and Loading data for the H2S-MEA system at $49^{\circ} \mathrm{C} \ldots 141$

D.5 Partial Pressure and Loading data for the H2S-MEA system $\ldots \ldots \ldots 142$

E.1 Recalculating solutions of Hallale(1998) using the MTA method . . . . 144 


\section{Nomenclature}

\section{Sets}

i Rich streams

j Lean streams

k Intervals

m Mass exchange units

p Components (sometimes ' $\mathrm{i}$ ' is used)

s Subnetworks

\section{Parameters}

$A C \quad$ Annualised cost of packed $\left(A C_{h}\right)$ or staged $\left(A C_{s t}\right)$ columns

$b_{i j} \quad$ Intercept of equilibrium line for component $p$ in lean stream $j$

$c_{j} \quad$ Annualised operating cost of MSA j

$H(i) \quad$ Henry's constant

$k_{y} a_{i} \quad$ Mass transfer coefficient for a match with rich stream $i$

$m_{i j} \quad$ Slope of equilibrium line for component $p$ in lean stream $j$ 
$U_{i j s} \quad$ Large positive value to ensure the existence of continuous variables when the binary variable $y_{i j s}$ is 1 . Sometimes $U_{i j m}$ is used. Its value is given by the minimum of the mass that can be transfered by the rich stream $i$ or accepted by lean stream $j$.

\section{Variables}

$A_{i j s} \quad$ Adsorption factor for a match $i j s$

$A D F_{i j s} \quad$ Average driving force for a match $i j s$

$b i n_{i j s} \quad$ Binary variable or binary variable-approximation to denote the selection of the form of the Kremser equation

CAP Capital cost of a mass exchanger network

$g_{i j s} \quad$ Flow of rich stream $i$ from $\left(g_{i j s}^{I}\right)$ an initial splitter, through $\left(g_{i j s}^{E}\right)$ an exchanger and to $\left(g_{i j s}^{O}\right)$ a final mixer of the stream

$g_{j j^{\prime} s s^{\prime}}^{B i} \quad$ Bypass flow of rich stream $i$ from match $(i j s)$ to match $\left(i j^{\prime} s^{\prime}\right)$

$H_{i j s} \quad$ Overall height of a packed column

$H T U_{i j s} \quad$ Height of a theoretical equilibrium stage in a packed column

$l_{i j s} \quad$ Flow of lean stream $j$ from $\left(l_{i j s}^{I}\right)$ an initial splitter, through $\left(l_{i j s}^{E}\right)$ an exchanger and to $\left(l_{i j s}^{\circ}\right)$ a final mixer of the stream

$l_{i i^{\prime} s s^{\prime}}^{B j} \quad$ Bypass flow of lean stream $j$ from match $(i j s)$ to match $\left(i^{\prime} j s^{\prime}\right)$

$L_{j} \quad$ Flow of lean stream $j$ through a mass exchange network

$m(i) \quad$ Molality of a species $i$,

$M_{i j s} \quad$ Mass of a shell of a packed columns

$M e_{i j s} \quad$ Mass exchange load of match (ijs)

$N s t_{i j s} \quad$ Number of theoretical plates or stages in an extraction column or a mixer-settler $N T U_{i j s} \quad$ Number of theoretical equilibrium stages in a packed column 
$O P C \quad$ Operating cost of a mass exchange network

$P \quad$ Pressure

Pw Water vapour pressure

T Temperature

TAC Total annualised cost of a mass exchanger network

$V_{i j s} \quad$ Volume of a staged vessel

$W_{i k}^{R} \quad$ Mass load of rich stream $i$ can transfer to a lean stream

$W_{j k}^{S} \quad$ Mass load that lean stream $j$ has the capacity to accept from a rich stream

$W_{i j m} \quad$ Mass that can be transfered between streams $i$ and $j$ in match $m$

$x_{i j s} \quad$ Composition of a lean stream $j$ prior $\left(x_{i j s}^{I}\right)$ and after $\left(x_{i j s}^{O}\right)$ match $i j s$

$x_{j}^{T} \quad$ Final composition of lean stream $j$ from a mass exchange network

$y_{i j s} \quad$ Composition of a rich stream $i$ prior $\left(y_{i j s}^{I}\right)$ and after $\left(y_{i j s}^{O}\right)$ match $i j s$

$Y r I_{i j s} \quad$ Driving force in terms of composition difference at the inlet of the rich stream at the unit (ijs)

$Y_{r O} O_{i j s}$ Driving force in terms of composition difference at the outlet of the rich stream at the unit $(i j s)$

$Y_{i j s} \quad$ Binary variable (sometimes $E_{i j m}$ is used) to denote the existence of a match between rich stream $i$ and lean stream $j$, in subnetwork $s$ (or in unit $m$ in the case of $E_{i j m}$ ). Not to be confused with the composition of a lean stream, $y_{i j s}^{I}$ or $y_{i j s}^{O}$.

$Z \quad$ Objective function 


\section{Greek letters}

$\alpha \quad$ liquid phase acid gas loading, mole acid gas/ mole amine

$\beta \quad$ The Debye-Huckle constant for the interaction of component $a$ with component b

$\beta_{i j s} \quad$ Feasibility variable or constant for the quadratic-constrain logic for use with the Kremser equation

$\gamma$

Activity coefficient

$\delta$

Residual mass exchange load to an interval $k$ from an interval $k-1$

$\varepsilon$

Minimum composition difference

$\rho \quad$ Density

$\tau$

Residence time per equilibrium stage

$\phi \quad$ fugacity coefficient 


\section{Chapter 1}

\section{Introduction}

\subsection{Process Synthesis}

Process synthesis is concerned with the systematic development of process Howsheets that transform available raw material into desired products and which meet specified performance criteria of maximum profit or minimum cost, energy efficiency, maximum rawmaterial recovery, minimum waste production and good operability. During this development the engineer is faced with many questions regarding the choice of process units, the inter-connectivity of the selected units and the optimal operating conditions. The units of choice that are available to the engineer are those within the reactor, separation and recycle, heat recovery and utility systems. A traditional approach to synthesising a process consisting of all or some of these units is to start the design process at the reactor system (the hub of the chemical process) and progress sequentially towards the utility systems. Figure1.1 illustrates the hierarchical levels of the synthesis process.

Within each subsystem, one calls on heuristics and thermodynamic insights to evaluate the potential of the anticipated flowsheet. The designer is continually involved throughout the synthesis process and has confidence in this type of flowsheet even though the synthesis procedure does not show how the various evaluation tasks at the different levels, when put together, affect the overall process design objectives.

There has been a growing interest to synthesise process flowsheets using a mathematical programming (MP) framework instead of the expert-system dependent hierarchical approach. The MP framework is based on the assumption that given the process units, their 


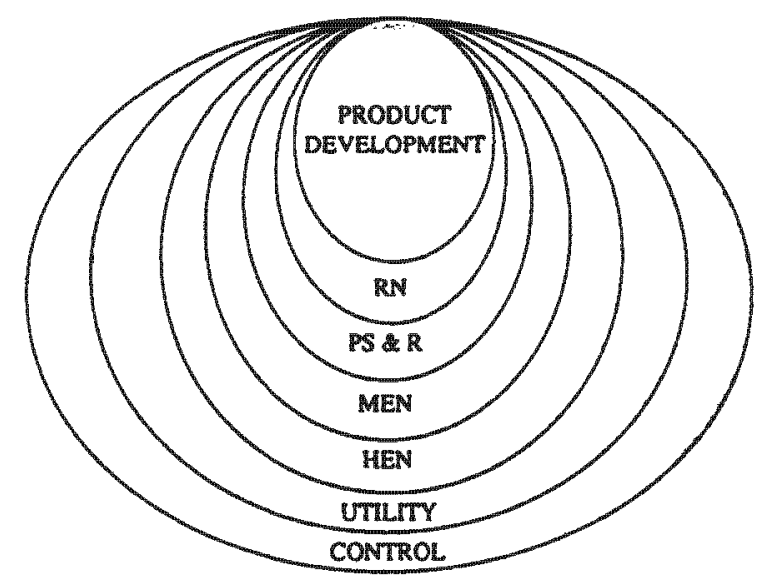

RN Reactor Network

PS\&R Primary separation and Recycle

MEN Mass Exchange Network

HEN Heat Exchange Network

Figure 1.1: Hierarchal levels of the synthesis process

connectivity and operating conditions, it is possible to formulate a representation that will encompass all process flowsheet alternatives from which the optimal flowsheet can be extracted. The generated representation can be simple or detailed in terms of both its layout and its mathematical description. The mathematical description is a collection of a set of variables, equations, constraints and optimisation objectives. The variables can be linearly or non-linearly related due to, for an example, non-linear chemical reaction kinetics or mass balance relations. The variables can also include discrete or integer variables that model discontinuous cost functions and the existence or non-existence of process units in the flowsheet.

In addition to the questions that the hierarchical designer must answer, the MP is faced with questions regarding the non-linearity, concavity and discontinuity of the model equations, the availability of a stable optimiser and a good initial solution. The confidence that one can place in the optimality of solutions obtained through the use of a less designerinvolved method needs also to be addressed. Despite these increased challenges, the MP framework is now accepted as a way forward in overall process synthesis since it has the potential to address the interaction between the subsystems of a process of any size. 
The boundaries between the insight-based and mathematical programming approaches are blurred by some similarities and cross-over of concepts from one approach to the other. Within the insight-based method, various heuristics can be automated and the evaluation of factors that affect the selection of the final flowsheet can represented by penalty functions, weights or indices in the objective function of the automation process. In a similar way, within the MP framework, the number of process alternatives that should be considered can be minimised according to physical and thermodynamic insights. In this way, both the MP power and thermodynamic insights are used to generate optimal flowsheets for the chemical industry.

\subsection{Synthesis of sub-systems}

In the early 1970s systematic methods for synthesising process flowsheets that met stringent energy requirements were emerging in the field of process synthesis. An important contribution from this period was the notion of Heat Exchange Network Synthesis (HENS) - a subsystem in the overall process synthesis that has gained a lot development and application in both academia and industry. HENS research brought forth the now so often used synthesis tool, Pinch Technology. Pinch Technology is based on two synthesis steps: a pinch analysis step and a flowsheet synthesis step. The pinch analysis step involves specifying a minimum energy transfer driving force to locate the operating conditions where there is constrained energy transfer and determine the minimum energy requirements ahead of any detailed design. In the flowsheet synthesis step, the conditions at the location where there is constrained energy transfer (the pinch point) are used to separate the energy streams into design regions. Within each region, heuristics and thermodynamic insights are used to design a network of heat exchangers that will meet the targeted conditions from the first step. The flowsheet synthesis process is evolutionary in nature and may sometimes require the pinch location to be disregarded in exchange for flowsheets that have fewer or reasonably-sized exchangers.

Parallel to the developments of targeting methods in HENs, other areas in process synthesis were beginning to adopt the approach of determining targets ahead of detailed design. As a result, methodologies for pinch analysis were and have been extended to the synthesis of utility networks and mass exchange networks (MENs). Attainable regions were introduced in reactor, primary separation and recycle networks to locate, ahead of design, the feasible operating regions of the anticipated flowsheet. 


\subsection{Pinch Analysis for MENS}

Following the extension of pinch technology to mass exchanger networks by El-Halwagi and Manousiouthakis (1989) significant advances have taken place to bring the use of this technology in mass exchange network synthesis to the same level as that of heat exchange network synthesis. In mass exchanger network synthesis, the transfer of mass from a set of rich streams to a set of lean streams is optimised. At first thermodynamic insights were used to set the targets for the minimum utility cost. The network design stage would be an iterative process of seeking a network that would meet the targets at the most economic capital cost - a network that featured a minimum number of mass exchangers. Recently, the introduction of capital cost targets in mass exchanger network synthesis have made it possible to trade-off operating and capital costs without having to evaluate many completed designs. These capital cost targets are discussed in Chapter 2. Targets have the advantage of preventing one from carrying out many designs before deciding on the best flowsheet. They provide the designer with confidence that a design is performing as well as it could. The graphical nature of the pinch approach is limited when large problems are considered and this necessitates the use of mathematical programming methods.

\subsection{Mixed integer non-linear programming}

Most chemical engineering problems can be modelled as mixed integer non-linear programming (MINLP) problems. MINLP problems are non-linear optimisation problems that involve integer variables in addition to continuous variables. These integers are normally used to model the existence or non-existence of units, sequences of events or alternative options. The continuous variables are used to model the input-output and interaction relationships among individual units or operations and different interconnected systems. The common applications of MINLP in chemical engineering include the design, scheduling, and planning of batch processes; the interaction of design and control; molecular product design; facility location and allocation; facility planning and scheduling and the topology of transport networks (Floudas, 1995).

The structure of the MINLP model is of the following form: 


$$
\begin{aligned}
\min _{x, y} f(x, y) & \\
\text { s.t. } h(x, y) & =0 \\
g(x, y) & \leq 0 \\
x \in X & \subseteq \Re \\
y \in Y & \text { integer }
\end{aligned}
$$

where $x$ is a vector of $n$ continuous variables, $y$ is a vector of integer variables, $h(x, y)=0$ are $m$ equality constraints, $g(x, y) \leq 0$ are $p$ inequality constraints, and $f(x, y)$ is the objective function. There exist both linear and non-linear terms in the constraints and objective function.

The design of mass exchanger networks using an MINLP approach takes three main steps. Firstly a framework or a superstructure is developed that will include within it all process units and connectivity alternatives. There is no generalised superstructure except that it should embed the optimum solution. Secondly, the superstructure is described by mathematical equations mostly representing mass balances around processing units and through the flowsheet, feasibility constraints and structural generation equations using the integer variables. The existence of a unit is defined by an integer value of 1 while its absence is described by an integer value of 0 . Finally the model is transfered to an MINLP solver from whose output an optimal solution is obtained. Note that the use of the integerslando is a special and common way of using integers in current commercial solvers of MINLP models. The integersland0 are referred to as binary variables.

Mathematical models have the advantage of being automated, but provide little scope for user interaction, require expensive computation, non-convexities that appear in the model due to non-linear cost laws could exclude the feasible region of existing algorithms, the problem size grows exponentially with the number of binary variables, and there is no guarantee that the solutions generated by current commercial solvers for solving nonconvex models are global.

\subsection{Motivation and Goals}

Grossmann and Daichendt(1996) in an assessment of process synthesis highlighted the following areas as the remaining challenges within process synthesis: 
1. The integration of heuristic search, optimisation and targeting approaches in such a way that on the one hand the integration is conceptually consistent and rigorous, and on the other hand it exploits the strengths of each approach;

2. The development of synthesis techniques that are able to handle complex process models;

3. The systematic generation of superstructures for process flowsheets that may involve different types of processing tasks or where non-linearities give rise to infeasible solutions that are not easy to predict;

4. The mapping of target and aggregated superstructure models onto detailed process flowsheets;

5. The reduction of combinatorial searches without sacrificing optimality;

6. How to develop computational frameworks that can handle multiple objectives such as operability, safety and environmental aspects;

7. The need for robust global optimisation methods that are relevant to process synthesis problems; and

8. How to integrate the selection of chemical pathways in process synthesis.

The option of combining the heuristic search, optimisation and targeting approaches is suggested. The heuristic search uses engineering knowledge and involves successive refinement and generation of alternatives. The optimisation approach provides a systematic framework for modelling and simultaneous optimisation, and also for providing automated capabilities for synthesis problems. Targeting methods such as pinch analysis provide useful physical and geometric insights to predict features that a feasible solution should exhibit. This thesis considers challenge 1 and aims to integrate pinch insights with the optimisation approach for the synthesis of mass exchange networks. In doing so, it is hoped that this will help reduce combinatorial searches of the optimisation methods (challenge 5) while the learning from modelling MINLP problems will necessitate the development of methods to handle complex models (challenge 2). In general the marriage of these two powerful MENS tools (MINLP and PINCH) will provide the much needed confidence in MINLP solutions and expose novel structures that would otherwise have not been conceivable through pinch methods alone. 


\subsection{Thesis Overview}

In developing the hybrid tools, the thesis will rely heavily on the work of Hallale, (1998) and the MINLP model of Papalexandrietal.,(1994). The contributions from this thesis will be:

1. The provision of a clearer understanding of the complexities involved in using MINLP models to synthesise MENs;

2. The development of a solution algorithm that highlights opportunities for the integration of the PINCH and MINLP tools;

3. The development of logical operators for use in the selection of appropriate sizing models;

4. The formulation of an analytical method to approximate the number of stages in a separation column that features non-linear equilibrium functions; and

5. The integration of the process of predicting VLE data into the traditional task of synthesising MENs when, during the problem statement, the assumption of available equilibrium functions is relaxed.

These developments are thus highlighted as follows:

\section{Chapter 2}

After introducing a brief background on the concepts of mass exchange networks, Chapter 2 reviews the literature that is relevant to the developments in this work. Different mass exchange design methods are discussed with emphasis on the pinch design method and the mixed integer non-linear programming method. The last part of the section summarises the latest efforts towards the development of hybrid design methods within the area of process synthesis and set out the foundation upon which the extensions to mass exchange network synthesis are based.

Computational issues are discussed at the end of Chapter 2. Here the reader is introduced to the two major algorithms that are currently used to in the field of process synthesis to solve large MINLP models, the commercial solvers that are integrated with these algorithms and the modelling environments in which the MINLP models may be implemented. 


\section{Chapter 3}

Chapter 3 introduces two fundamental tools that are necessary for the sizing of trayed or stages vessels: the logical operators and the approximation of the McCabe-Thiele method for the determination of the number of stages when equilibrium curves in a MENS problem are non-linear.

\section{Chapter 4}

This chapter focuses on the MINLP model selected for the illustration of the hybrid procedure. Emphasis is placed on the modifications in the equations, boundaries and method of solution of the Papalexandri model. The second part of this chapter re-works literature examples and compares the results with those previously reported by El-Halwagi and Manousiouthakis (1989), Papalexandri et al., (1994), Hallale (1998), Wang and Smith (1996) and Comeaux (2000) .

\section{Chapter 5}

A case study is solved in Chapter 5 to illustrate the integration of the prediction of thermodynamic parameters into the synthesis task. Equilibrium functions differ according to the temperature, pressure and concentration conditions of the process streams. In this context, the assumption of a pre-assigned single equilibrium function in the definition of a MENS problem needs to be relaxed and the generation of the equilibrium function become part of the synthesis process. In addition, a range of equilibrium functions is generated for the same process MSA because of the different operating conditions for the matching rich streams.

\section{Chapter 6}

Chapter 6 will draw important conclusions from this work. Firstly, the highlights of this thesis will be presented, then the possibilities of future work will be considered. 


\title{
Chapter 2
}

\section{Literature Review}

\author{
Nur die Fulle fuhrt zur Klarheit \\ Und im Abgrund wohnt die Wahrheit \\ Niels Bohr (1885 - 1962)
}

\subsection{Introduction}

This section reviews the literature that is relevant to the developments in this thesis. Prior to the review, it is instructive to define the terms and jargon that is commonly used in mass exchanger networks synthesis. The review section itself is divided into 3 main parts: the synthesis methods used in the pinch analysis approach, current MINLP developments in MENS and a brief introduction into the work already done on the integration of different synthesis methods for MENs.

For completeness the end of the review introduces the reader to the modelling and computational issues in mathematical programming especially those that are relevant to this thesis.

\section{Mass exchange networks}

Mass exchange networks (MENs) are used in the chemical industry to recover high-valued components or to reduce the waste generated by the plant to an acceptable level in the most economical way. Examples of mass exchange operations are absorption, desorption, 
adsorption, liquid-liquid extraction, leaching and ion exchange. These are direct-contact, counter current mass transfer operations that use a mass separating agent to preferentially transfer a certain species from a set of rich streams to a set of lean streams or mass separating agents (MSAs). The systematic generation of a cost effective network of these units constitutes the task of mass exchange network synthesis (MENS).

\section{Rich streams}

These are streams in a processing facility that carry the desired components to be removed for waste minimisation purposes or for the recovery of products and raw material. Thus the rich streams are streams that are rich in the transfered component - whether useful or waste material.

\section{Lean streams}

Lean streams are available in a plant (process MSAs) or externally (external MSAs) and they carry less or none of the component to be transfered from the rich streams. They are used to remove the desired component from the rich stream via mass transfer principles. The objective in the mass transfer process, is that as much as possible of the process MSAs must be utilised before embarking on purchasing external MSAs.

\section{The objective function}

All MENS procedures are optimisation processes where the economic benefit of a process is evaluated. Traditionally, cost has been used as the criterion to determine the best mass exchange flowsheet. Current trends aim to include objectives that evaluate the environmental, safety, control and operability aspects of a process as well. These aspects may be related to cost or expressed as a weighted index in the objective function.

Against this backdrop it is now possible to define the problem statement for the synthesis of mass exchange networks. 


\section{Problem statement}

El-Halwagi (1997) gives the following problem statement for the synthesis of mass exchange networks:

Given a number $N_{R}$ of waste (rich) streams and a number $N_{S}$ of mass separating agents (MSA) or lean streams, it is desired to synthesise a cost-effective network of mass exchangers that can preferentially transfer certain undesirable species from the waste streams to the MSAs. Given are also the flowrate of each waste stream, $G_{i}$, its supply (inlet) composition $y_{i}^{s}$, and its target (outlet) composition $y_{i}^{t}$, where $i=1,2, \ldots, N_{R}$. In addition, the supply and target compositions, $x_{j}^{s}$ and $x_{j}^{t}$, are given for each MSA, where $j=1,2, \ldots, N_{S}$. The flowrate of each MSA is unknown and is to be determined so as to minimise the network cost.

The candidate lean streams can be classified into $N_{S P}$ process MSAs and $N_{S E}$ external MSAs (where $N_{S P}+N_{S E}=N_{S}$ ). The process MSAs already exist on the plant site and can be used for the removal of undesirable species at a very low cost. The flowrate of each process MSA that can be used for mass exchange is bounded by its availability in the plant, i.e.,

$$
L_{j} \leq L_{j}^{c}, \quad j=1,2, \ldots, N_{S P}
$$

where $L_{j}^{c}$ is the flowrate of the $j^{\text {th }}$ MSA that is available in the plant. On the other hand, the external MSAs can be purchased from the market. Their flowrate will be determined according to the overall econornic considerations of the MEN.

Figure2.1 illustrates a mass exchange unit with the operating variables corresponding to the problem statement.

\section{Mass transfer complexities}

The similarities between heat and mass transfer have helped to advance the developments in mass exchange networks towards those of heat exchanger networks, HENs. These synthesis technologies are based on clearly identifiable commodities, their source and destination 


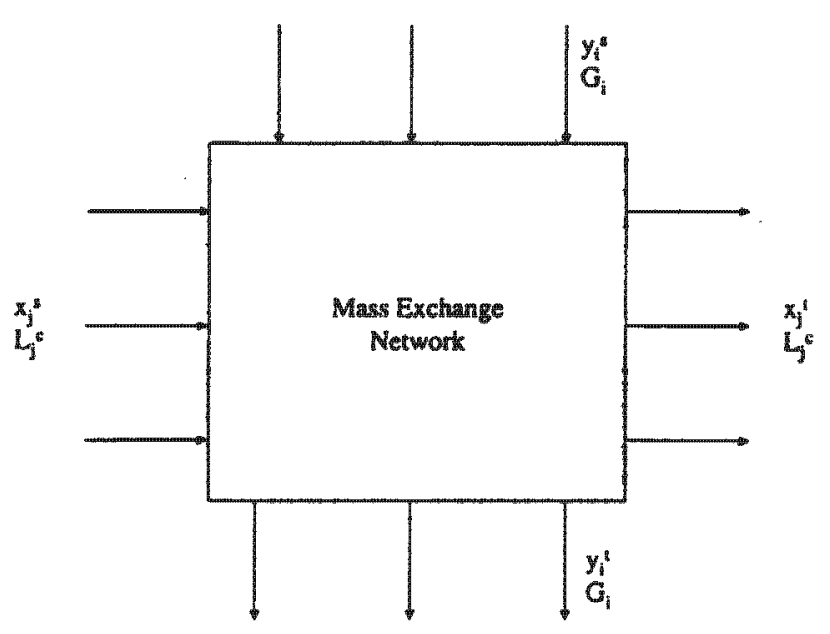

Figure 2.1: A mass exchange network

and the equilibrium criteria for a feasible transfer (see Table2.1). In MENS, phase equilibrium characteristics of the solute-solvent system govern the distribution of solutes between the rich and lean phase. A complexity in MENS is brought about by the mechanism of transport. During mass transfer many conserved species can be transfered to the lean stream unlike in HENS where energy is the only transfered commodity.

Table 2.1: The comparison of HEN and MEN technology

\begin{tabular}{|c|c|c|}
\hline Concept & MEN & HEN \\
\hline \hline Transported material & several conserved species & energy \\
\hline Equilibrium & solute-solvent phase equilibrium & temperature \\
\hline Driving force & composition difference & temperature difference \\
\hline Source & rich streams & hot streams \\
\hline Sink & lean streams & cold streams \\
\hline
\end{tabular}

The identified subtle differences suggest that even if the mass and heat transfer are analogous, there can be no simple or direct translation of the HENS technology to MENS. Nevertheless a lot of MENS research has benefited from the many developments in HENS.

\subsection{Pinch Technology}

Pinch Technology has been successfully applied in industry and is a well accepted synthesis technique for HENs. It's history dates back to the 1970's and 1980's where it has been 
largely responsible for the many improvements in energy savings campaigns. Based on fundamental thermodynamics, Pinch Technology is used to systematically analyse heat flows through industrial processes and to assess the trade-off between energy consumption and capital investment. It provides a two-step guide in the generation of design options for the reduction of energy consumption: In the first step the designer can determine design targets based on thermodynamic insights without him or her committing to a specific design. The second step involves the use of heuristics and physical insights to evolve an initial flowsheet to meet the targets set in the first step. Details of the developments of thermal pinch are discussed in reviews by Gundersen and Naess(1988) and Linnhoff(1993)

In the late 1980's pinch analysis was finding use in mass transfer for the screening of candidate MSAs while incorporating thermodynamic and economic considerations(El-Halwagi, 1989). The early work on mass transfer pinch was setting MSA targets and then designing a network by minimising the number of transfer units in the process. This objective was an extension from HENS and was justified by the need for less pipe-work, foundations, maintenance and instrumentation and subsequently minimum capital expenditure. Other targets that have come to find use in MENS are summarised in Table2.2 along with those developed in HENS.

Table 2.2: Existing targets in the HEN and MEN technologies

\begin{tabular}{|c|c|}
\hline MENS Targets & HENS Targets \\
\hline MSA cost & Utility costs \\
\hline Number of units & Number of units \\
\hline Capital cost & Capital cost \\
\hline Supertaregting & Supertargeting \\
\hline Retrofit targeting & Retrofit targeting \\
\hline
\end{tabular}

In supertargeting the MSA cost (operating cost) and capital cost are traded off against the minimum composition difference to determine a minimum Total Annual Cost target (TAC). These targets represent realistically attainable goals, based on thermodynamic principles and good use of driving force. The next section discusses briefly the process of obtaining operating and cost targets for subsequent inclusion in the supertargeting procedure. Although a detailed discussion on supertargeting is given in the PhD work of Hallale (1998), the review is necessary to streamline the developments in this thesis. 


\subsubsection{Operating cost targets}

The starting point for the determination of MSA cost targets is the selection of a minimum driving force or composition difference, $\varepsilon$, which ensures that the mass transfer between a rich and a lean stream is feasible. The driving force is the difference between the actual and equilibrium compositions of the transfered species. The composition of a lean stream, $j$, in equilibrium with a rich stream, $i$, is $x_{j}^{*}$ as defined by the equilibrium relation:

$$
y_{i}=f\left(x_{j}^{*}\right)
$$

The $\varepsilon$ is the approach to equilibrium in a lean stream and is a measure of the driving force for mass transfer. For a feasible mass transfer between the $i^{\text {th }}$ and $j^{\text {th }}$ streams, the maximum value of $x_{j}$ is given by:

$$
x_{j}=x_{j}^{*}-\varepsilon
$$

Many applications involve dilute systems thus the equilibrium function $f\left(x_{j}^{*}\right)$ is assumed to be linear according to Equation2.3.

$$
\begin{aligned}
y_{i} & =m_{j} x_{j}^{*}+b_{j} \\
\text { or } x_{j}^{*} & =\frac{y_{i}-b_{j}}{m_{j}}
\end{aligned}
$$

Similarly, the equilibrium composition of a rich stream is given by Equation2.4.

$$
\begin{aligned}
y_{i}^{*} & =m_{j} x_{j}+b_{j} \\
\text { or } x_{j} & =\frac{y_{i}^{*}-b_{j}}{m_{j}}
\end{aligned}
$$

The composition differences in the rich and lean phases are related through the equilibrium constants as follows:

$$
\Delta y=m_{j} \Delta x
$$




$$
\Delta y_{\min }=m_{j} \varepsilon
$$

The transformation in Equation2.7 was significant in the work of (Hallale, 1998) where the capital cost targets depended on the availability of a common parameter to relate the two composition types, $x_{j}$ and $y_{i}$ in a similar fashion to temperature ( $T_{C o l d}$ or $\left.T_{H o t}\right)$ in HENS. It is now possible to detail the MSA cost targeting procedure for a selected $\varepsilon$ or $\Delta y$. $\Delta y$ is used to represent the driving forces when targeting for capital costs. It becomes more useful if the problem that is being considered involves more than one MSA on each side of the pinch location and a single scale for $x$ (El-Halwagi, 1989) can no longer be used.

\section{Rich composite curve}

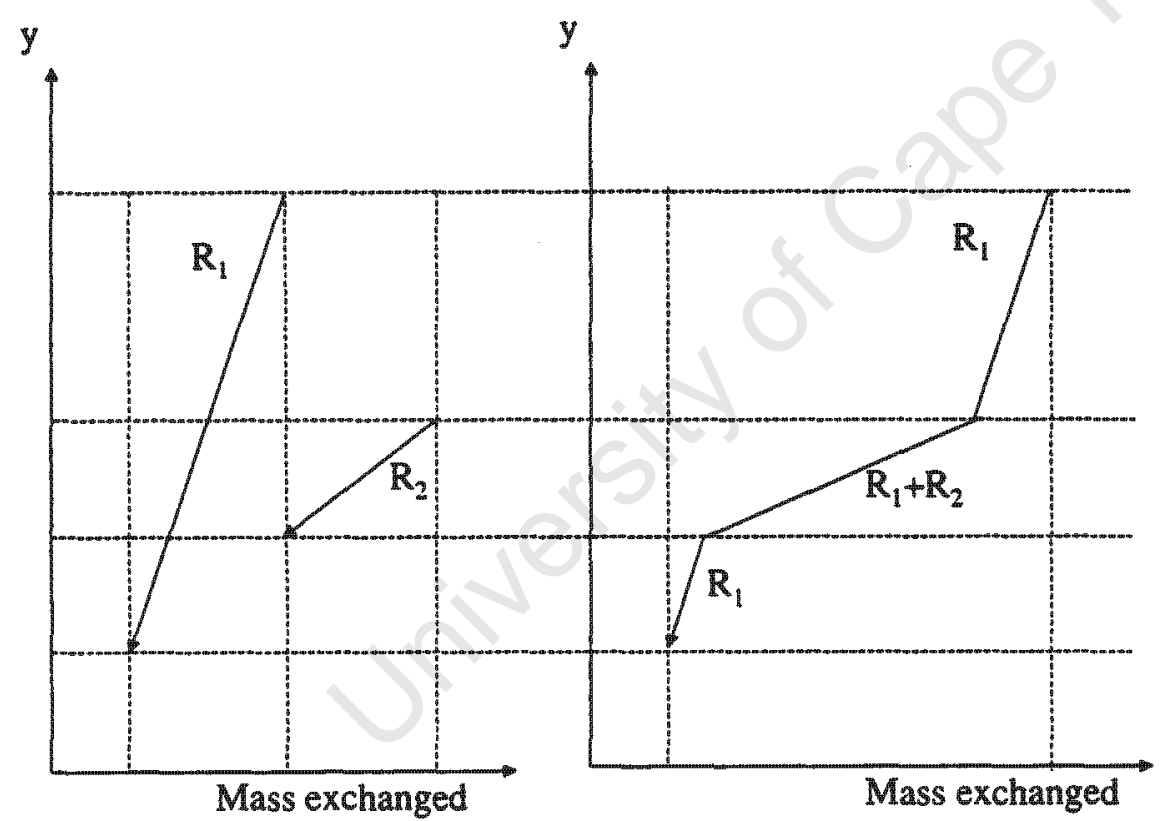

Figure 2.2: Construction of the rich composite curve

In a system having $N_{R}$ rich streams, the supply and target compositions of each of the rich streams correspond to interval boundaries or points of inflection on a plot of composition versus cumulative mass as in Figure2.2 for two rich streams. This rich composite curve is analogous to the hot composite curve in HENS. The inverse of the slope in each interval is equal to the total rich stream flowrate $\left(G_{i}\right)$ in that intervalk. When relatively small composition-changes are required or if some counter-diffusion is assumed to occur, the 
total flowrate of every stream is assumed to remain constant during mass transfer. In the case where stream flowrates change significantly, the flowrate and composition of inert components in each rich stream can be used to construct the composite curves.

\section{Lean composite curve}

For $N_{S}$ lean streams, the composition curve is generated by first converting each of the supply $\left(x_{j}^{s}\right)$ and target $\left(x_{j}^{t}\right)$ compositions of the lean streams to a corresponding $y^{*}$ value using Equation2.4 and then plotting all the process MSAs together. The flowrates of these MSAs are placed at their maximum, $L_{j}^{c}$. Figure2.3 illustrates the construction of the lean stream equilibrium composition versus cumulative mass plot for two process MSAs. Note that instead of using $y$ or $y^{*}$ for the vertical axis, $x$ and $x^{*}$ can be used as well.

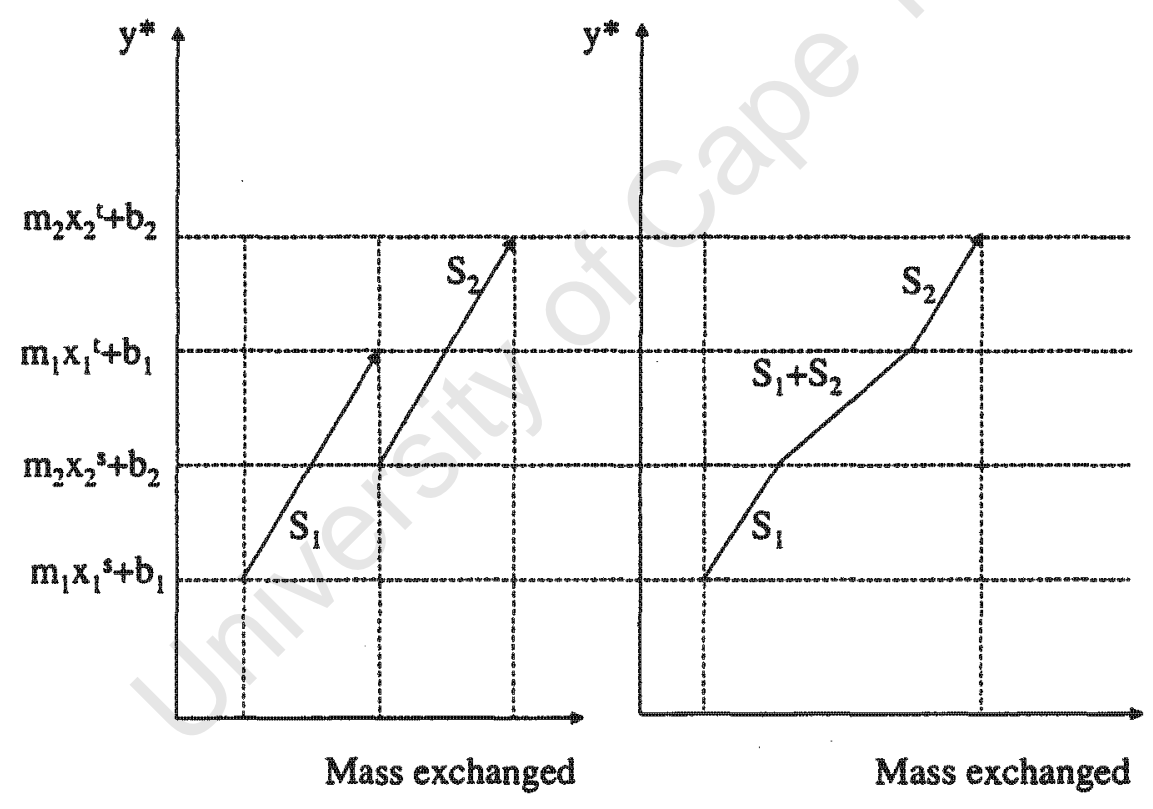

Figure 2.3: Construction of the lean composite curve using $y^{\prime \prime}$ compositions.

\section{The pinch curve}

Next, both the rich and lean stream composite curves are drawn on the same axes and the lean curve is shifted until it is $\Delta y_{\min }$ units away from the rich curve (see Figure2.4). The point where this minimum composition difference is located, is called the mass transfer 
pinch and it represents the thermodynamic bottleneck to mass transfer between process streams. The pinch point decomposes the problem into two regions: that containing all streams or parts of streams richer than the pinch composition (above the pinch) and the region containing all streams or parts of streams leaner than the pinch composition (below the pinch).

The distance by which the lean composite curve extends past the rich composite curve shows the excess capacity of the process MSAs to remove mass. This excess capacity cannot be used to remove the remaining load from the rich stream because it is thermodynamically infeasible. The excess capacity can however be eliminated by lowering the flowrate and/or outlet composition of one or more of the process MSAs. The way in which this reduction procedure has to be carried out is left to the designer. In Hallale(1998) the option of reducing the lean stream is used and it is the stream that has the highest inlet equilibrium composition $\left(y_{i n}^{*}\right)$ whose flowrate gets reduced. It is indeed simpler in the targeting procedure to alter the flowrate of the process streams than the compositions as changes to the latter will require changes to the equilibrium compositions. The load to be removed by an external MSA is determined from the distance by which the rich composite curve extends past the lean composite curve.

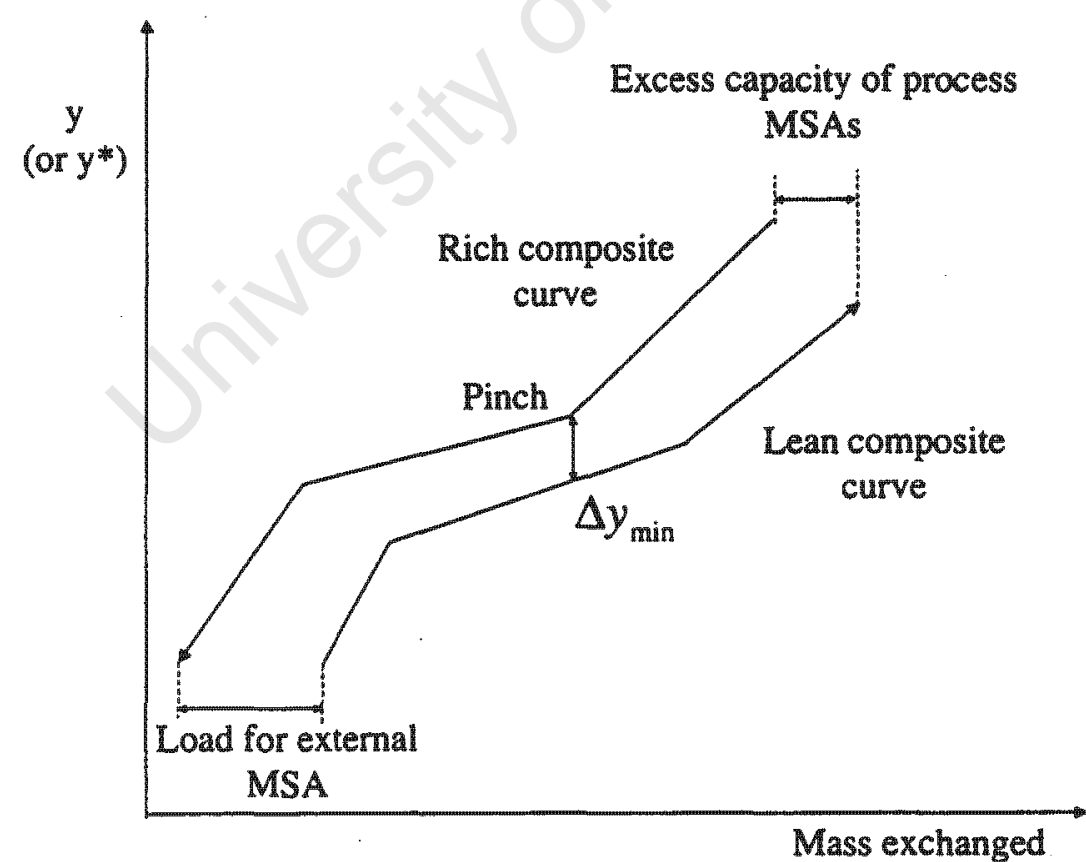

Figure 2.4: Combined composite curves to locate the pinch point and determine MSA. targets 


\section{The operating cost}

The outlet composition of the external MSA may be found from the supply composition of the lean stream with the lowest equilibrium composition $y^{*}$ or from pinching the external MSA against the rich composite curve. This ensures that not only is the mass balance feasible but that it is thermodynamically feasible for the external MSA to remove the load. If only the mass balance feasibility is considered, the external MSA would have been minimised by allowing its final composition to reach the target composition $\left(x_{j}^{k}\right)$ as it is often done in water pinch (Wang and Smith, 1994). This information can then be used to determine the minimum cost of MSAs for the particular $\varepsilon$ or $\Delta y_{\min }$. Thus for each minimum composition difference, the operating cost can be obtained as the sum of the product of the MSA flowrates multiplied by their unit cost and the number of operating hours in a year.

Apart from the graphical method of targeting for minimum operating costs, composite interval tables (El-Halwagi, 1989) and linear programming methods (El-Halwagi and Manousiouthakis, 990a) can be used as well.

\subsubsection{Capital cost targets}

Hallale (1998) proposed a method for the determination of capital cost targets. The mass transfer pinch plot is important in the determination of these targets. After determining the targets for the flowrates of the process and external MSAs, the composite curves are redrawn in perfect mass balance (see Figure2.5) - this time incorporating the MSA flowrate targets.

A composite operating line is drawn by plotting the $y$ values versus the $y^{*}$ values throughout the network. This $y-y *$ composite curve plot also incorporates a pseudo-equilibrium line,

$$
y=y^{*}
$$

The composition differences between the composite operating line and the pseudo-equilibrium line are the same as those along the mass transfer composite curves. This plot represents the driving forces, and operating and equilibrium lines for the entire network. The points of inflection on the composite operating line are used to partition the flowsheet into $k$ composition intervals. These intervals can have a number of stages or transfer units targets 


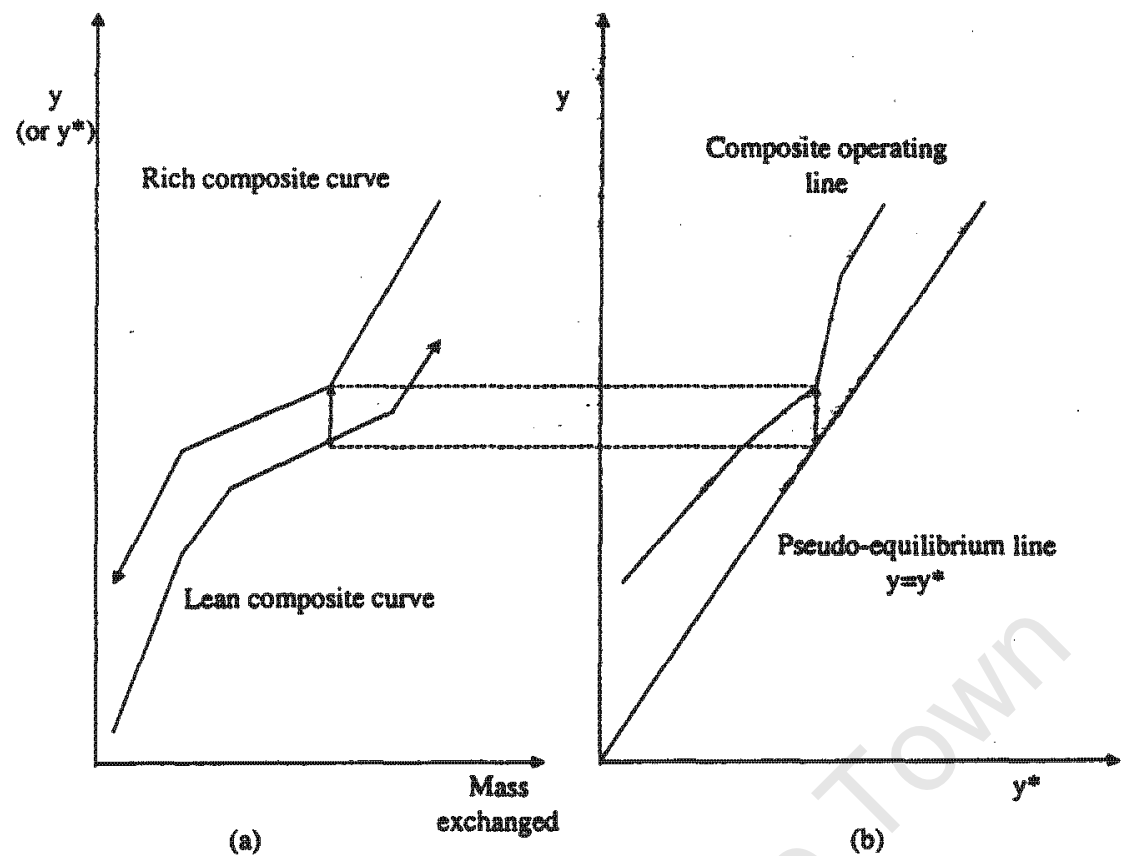

Figure 2.5: Construction of the $y-y^{*}$ curve for sizing exchangers

assigned to them. Each stream that makes up the composite curve in each interval will contribute to the sizing targets.

For stagewise exchangers, the number of stages for an interval can be determined by using the Kremser equation, provided the operating and equilibrium lines are linear.

$$
\begin{aligned}
N_{s t} & =\frac{\ln \left[\left(\frac{y_{i, \text { in }}-m_{j} x_{j, \text { in }}-b_{j}}{y_{i, \text { out }}-m_{j, j} x_{j, \text { in }}-b_{j}}\right)\left(1-\frac{1}{A_{i j}}\right)+\frac{1}{A_{i j}}\right]}{\ln A_{i j}} \\
& =\frac{\ln \left[\left(\frac{y_{i, \text { in }}-y_{i, \text { in }}^{*}}{y_{i, \text { out }}-y_{j, \text { out }}}\right)\left(1-\frac{1}{A_{i j}}\right)+\frac{1}{A_{i j}}\right]}{\ln A_{i j}}
\end{aligned}
$$

where

$$
A_{i j}=\frac{L_{j}}{m_{j} G_{i}}=\frac{\left(y_{i, \text { in }}-y_{i, \text { out }}\right)}{m_{j}\left(x_{j, \text { out }}-x_{j, \text { in }}\right)}
$$

and

$$
A_{i j} \neq 1
$$


When the absorption factor $A_{i j}$ in the numerator of Equation2.9is expressed in terms of composition, the Kremser Equation becomes less non-linear form as shown by Equation2.10 (El-Halwagi, 1997).

$$
N_{s t}\left(A_{i j}\right)=\frac{\ln \frac{y_{i, i n}-m_{j} x_{j, \text { out }}-b_{i j}}{y_{i, \text { out }}-m_{j} x_{j, \text { in }}-b_{i j}}}{\ln A_{i j}} A_{i j} \neq 1
$$

When $A_{i j}$ is 1 in Equations2.9or2.10, Equation2.11 is called upon to determine the number of stages.

$$
N_{s t}\left(A_{i j}\right)=\frac{y_{i, \text { in }}-y_{i, \text { out }}}{y_{i, \text { out }}-m_{j} x_{j, \text { in }}-b_{j}} \quad A_{i j}=1
$$

The absorption factor $A_{i j}$ for a component $p$ determines how readily component $p$ will absorb into the lean phase, where $L_{j}$ and $G_{i}$ are the lean and rich stream flowrates and $m_{i}$ is the slope of the equilibrium line for $p$. The slope of the composite operating line in each interval represents the absorption factor for that interval. This slope is given by Equation2.12.

$$
A_{i j}=\frac{L_{j}}{m_{j} G_{i}}
$$

When this factor is large, component $p$ will be absorbed more readily into the lean phase. The absorption factor is increased by increasing the lean stream flowrate. This increase leads to a reduction in the number of stages required to achieve a given separation. At high values of the absorption factor, the increase in liquid flowrate brings diminishing returns. An economic optimum is thus realised for different stream matches. Smith(1995) notes that the absorption factor must be greater that 1 for a high degree of solute removal with the economic potential lying in the range $1.2 \leq A_{i j}<2$. King(1980) proposed an absorption factor of around 1.3. In an $\mathrm{SO}_{2}$ removal problem solved by Alva-Argaez(1999) the constant value of 1.3 was used to size an exchanger. The resulting size of the unit was a 581 staged vessel with a corresponding unrealistic-height of $300 \mathrm{~m}$. Although this problem was not concerned with the minimisation of equipment size it, however, illustrates the importance of allowing the adsorption factor to be determined by the operating conditions of the final process.

When the absorption factor is unity, then the number of stages are determined by Equation2.11. Later, it will be shown that the success of using the appropriate Kremser Equation depends on the availability of logical operators that will help in switching between Equations2.10and2.11 
subject to the value of the absorption factor.

For continuous columns with straight operating and equilibrium lines, Treybal(1981) showed that the height of an exchanger is given as a function of the overall number of transfer units $N T U_{y}$ and the overall height $H T U_{y}$ of the transfer unit according to Equation2.13.

$$
H=H T U_{y} N T U_{y}
$$

In each composition interval $k$, the number and height of transfer units can be determined according to Equations2.14andB.0.3.

$$
\begin{aligned}
N T U_{y, k} & =\frac{y_{k, \text { in }}-y_{k, \text { out }}}{\Delta y_{l m, k}} \\
\Delta y_{l m, k} & =\frac{\left(y_{k, \text { in }}-y_{k, \text { in }}^{*}\right)-\left(y_{k, \text { out }}-y_{k, \text { out }}^{*}\right)}{\ln \left(\frac{\left(y_{k, \text { in }}-y_{k, \text { in }}^{*}\right)}{\left(y_{k, \text { out }}-y_{k, \text { out }}\right)}\right)}
\end{aligned}
$$

and

$$
H T U_{y, k}=\frac{M e_{k}}{S K_{y} a_{i}}
$$

where:

$\Delta y_{l m, k}$ is the log mean driving force

$M e_{k}$ is the mass that is transfered in an interval k

$K_{y} a_{i}$ is the overall mass transfer coefficient for the rich phase.

$S$ is the cross-sectional area of the mass exchange equipment

The information for completing the sizing calculations is available from the $y$-y* diagram illustrated by Figure2.5. An interesting aspect to note when determining capital cost targets is that the stream splitting rule assumes that each match in an interval would have the same value of $A_{i j}$ as the interval.

Hallale (1998) discusses other sizing models based on the mass of an exchanger shell or the volume of an exchanger. Whatever model is used, it is based on some transformation of Equations2.9and2.13. The details of these models are summarised in AppendixB. 


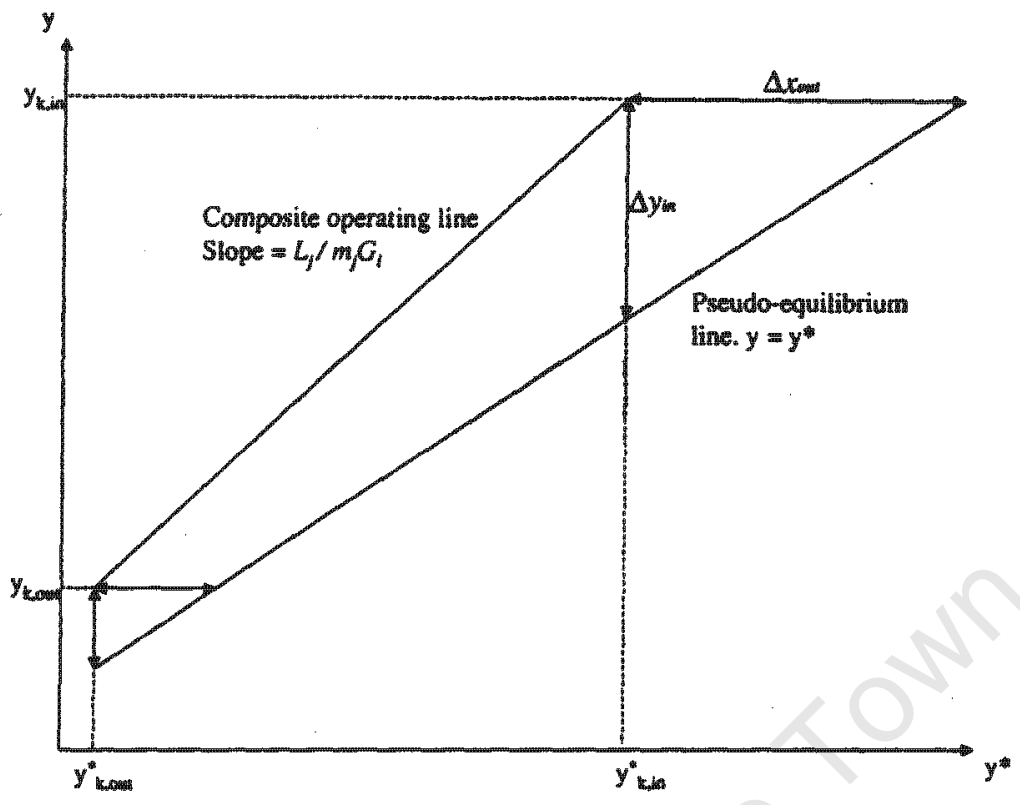

Figure 2.6: Exchanger sizing parameters from a y-y* curve plot

For a particular minimum composition difference, $\varepsilon$ or $\Delta y_{\min }$, the capital cost target can be obtained multiplying an appropriate costing function with an annualisation factor. This annualisation factor is mostly a function of the period within which the capital investment is to be recovered.

\subsubsection{Supertargeting}

Hallale (1998) showed how the capital and operating cost, through supertargeting, could be used to optimise the total annual cost (TAC) of a network before design. Supertargeting is a powerful tool that considers simultaneously, both the capital and operating costs for a given driving force. The MSA cost targets alone can provide the false expectation of a thermodynamically feasible network based on minimum utility cost whilst the same network may be having very large exchangers which are unrealistic and impractical to construct. The simultaneous concept refers to the selection of targets that are based on the contributions of both capital and operating cost. It does not refer to the simultaneous optimisation and design that is achieved by using MINLP methods.

In supertargeting the two costs are plotted together (seeFigure2.7) and for each $\varepsilon$ or $\Delta y_{\min }$ the TAC is determined by summing the annual operating and capital cost. The optimal $\varepsilon$ 
or $\Delta y_{\min }$ is then the one that gives a minimum TAC.

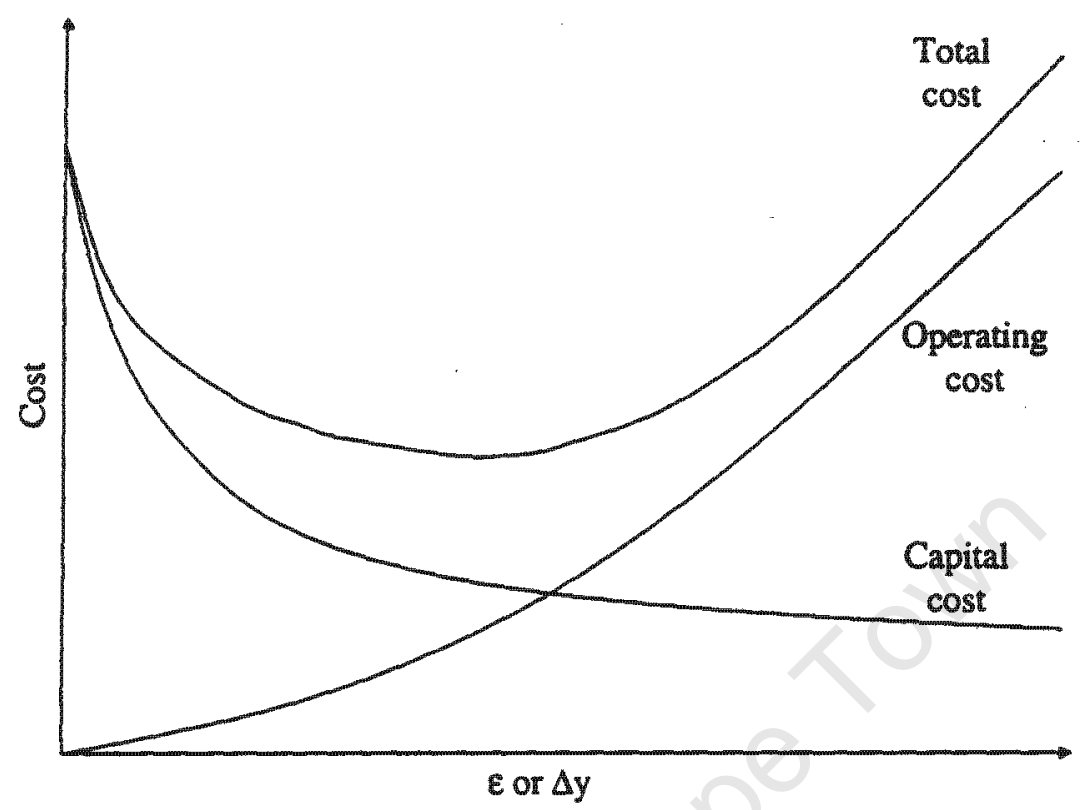

Figure 2.7: Supertarget diagram for the selection of an optimum minimum composition difference

\subsubsection{Design from supertargets}

With the selected optimal $\varepsilon$ or $\Delta y_{\min }$, a network design is carried out on the grid diagram (Figure2.8).

The grids in Figure2.8 correspond to the rich stream supply and target compositions. These compositions are placed in decreasing order of magnitude from the supply composition of the most rich rich-stream to the target composition of the most lean rich-stream. The lean stream compositions are shown as $y^{*}$ values. The rich streams are drawn running from right to left and lean streams in the opposite direction. Exchangers are represented as a pair of joined circles with the mass transfered shown underneath or tabulated for clarity. The placement of exchangers and evolution of the initial network to an optimal one is based on the network generation criteria of El-Halwagi and Manousiouthakis(1989) and Hallale(1998).

Also on the grid diagram, the pinch compositions found at the minimum TAC target are identified and the network is generated starting from the pinch point (for above and below 


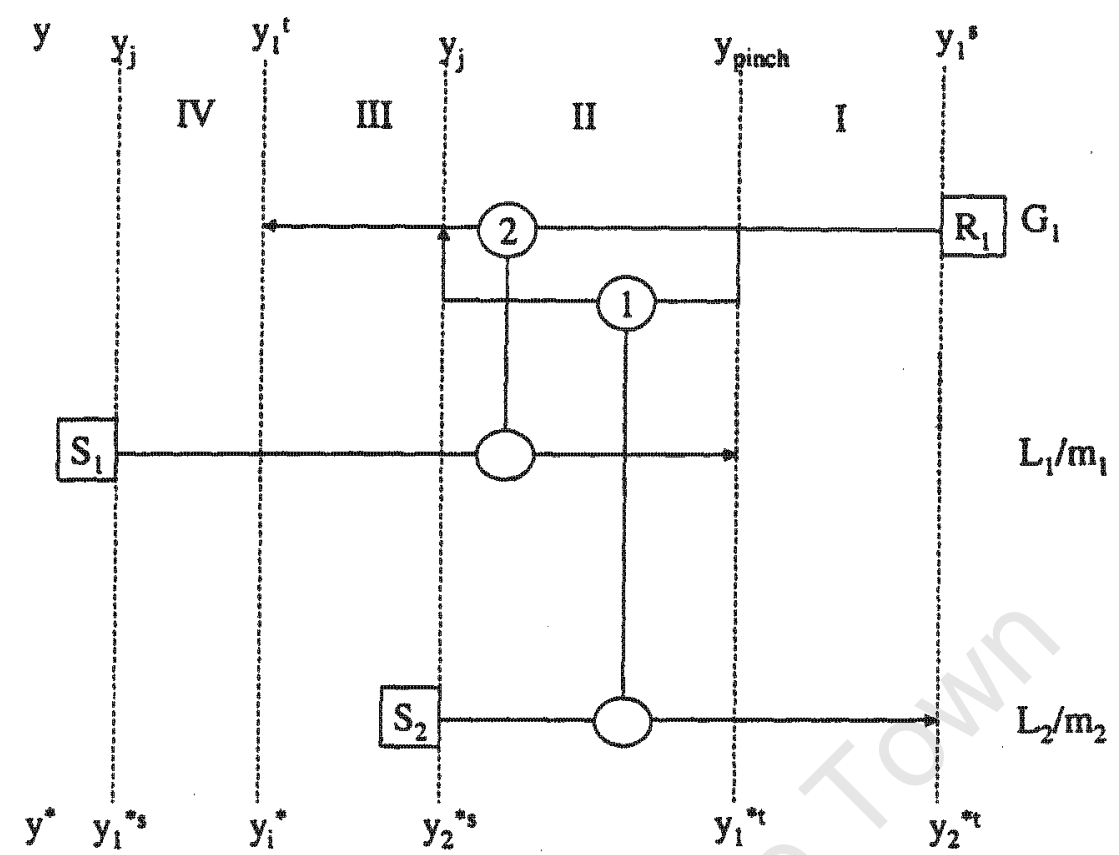

Figure 2.8: Grid diagram for capital cost targeting and network generation the pinch) and moving away from the pinch.

\section{The network design criteria}

Two categories of design rules are identified from the literature: those that ensure that the minimum MSA cost targets are met and are based on the thermodynamic pinch and stream population (El-Halwagi, 1989) and those ensuring that the minimum capital cost targets are met and are based on the correct use of driving force (Hallale, 1998). These rules are summarised below.

1. Stream population

Immediately above the pinch,

$$
N_{R, \text { above pinch }} \leq N_{S, \text { above pinch }}
$$

and immediately below the pinch

$$
N_{R, \text { below pinch }} \leq N_{S, \text { below pinch }}
$$




\section{Pinch enforcement}

If $\varepsilon$ or $\Delta y_{\min }$ are to be the minimum composition differences, the operating line and the equilibrium line must diverge away from the pinch. It thus becomes important to impose the following relationships for the matches that are immediately either side of the pinch.

$$
\left(\frac{L_{j}}{m_{j}}\right)_{\text {above pinct }} \leq G_{i, \text { above pinch }}
$$

and

$$
\left(\frac{L_{j}}{m_{j}}\right)_{\text {below pincls }} \geq G_{i, \text { below pinch }}
$$

3. The removal factor, $\left(\frac{L}{m G}\right)$ or driving force rule

In order to meet the capital cost target, each match should be selected such that its removal factor is similar to the removal factor of the composite operating line. The $y-y^{*}$ composite curve is called upon to evaluate this use of driving force. At the pinch point this rule is similar to the pinch enforcement rule. Away from the pinch the driving force rule allows the matches to be superimposed on the composite operating curve thus making the best use of the available driving force. The use of the removal factor to evaluate how a match uses the available driving force is simpler than placing a match on the $y-y^{*}$ composite curve. This observation has to be cautiously applied when a match spans a range of intervals and as a result has different slopes. In such cases, the designer can resort back to using the driving force plot.

\section{Remaining problem analysis}

Remaining problem analysis quantifies the penalty paid for selecting a match that leads to additional number of stages required over and above the target. This rule becomes important in this thesis as it illustrates an opportunity to include targets in the MINLP approach to mass exchange network synthesis.

Step 3 and 4 can be carried out without considering the pinch position. Once a near optimal solution is identified, mass-load paths ((El-Halwagi, 1997)) can be used to improve the flowsheet further. The basis for using mass-load paths is that an excess amount of an external MSA can be added to replace an equivalent amount of a process MSA with the end result being the elimination of exchangers without incurring additional operating cost. This is beacause the additional operating cost would be covered by the savings 
from the elimanated exchangers. El-Halwagi (1997) states that the extent to which the fixed cost of mass exchangers would decrease determines whether the additional operating cost is acceptable. This approach is thus similar to that of obtaining the final network from considering many flowsheets at different minimum composition differences, the only difference here being that mass-load paths are used and there are capital cost targets to evaluate the quality of the flowsheets generated.

\subsubsection{Pinch analysis summary}

\section{Potential of project ahead of design}

The mass transfer pinch and the operating cost and capital cost targeting methods are useful in determining a network's potential for effluent reduction, raw material recovery and total cost minimisation. The targets however, cannot predict the complexity of a flowsheet since they are determined according to a cost-based objective function.

\section{Involvement of the designer}

Pinch analysis provides a conceptual understanding of the driving forces of a process flowsheet. The designer can influence the network layout, through match selection, stream splitting and through use of personal experience. This continuous involvement of the designer also depends on the availability of graphical tools which aid in the decision-making process. Large and complex problems could be hindered by the difficulty in visualising design trade-offs.

\section{Trade-offs at targeting stage}

The options of how to reduce excess process MSA during the targeting for minimum MSA are the reduction of the lean stream flowrates and/or the target compositions. The tradeoff between composition reduction and flowrate reduction when no cost is associated with the process MSAs is not taken into account during the targeting stage. The choice of reducing the flowrate can have a negative impact on the capital cost whilst the reduction of the outlet composition of a stream could increase the total flowrate of the stream and affect matches at the lower composition spectrum of the design grid diagram. Once targets are selected, most pinch design examples from Hallale(1998) are based on fixed outlet 
compositions and process MSAs flowrates that are fixed at their target values. The only optimised variable is the external MSA flowrate and driving force for matches that are far away from the pinch point.

\subsection{Mathematical programming}

The synthesis of mass exchange networks using mathematical programming methods has been a subject of growing interest since the introduction of the mass transfer pinch. The programming methods have played major roles in automating the determination of mass transfer utility targets and minimising the number of mass exchange units in a flowsheet (using linear and mixed integer linear programming, El-HalwagiandManousiouthakis,1990a) and in determining the best operating conditions of a fixed flowsheet (using non-linear programming, GuptaandManousiouthakis,1993; LeeandPark,1996) . Mathematical programming has also been used to simultaneously generate a mass exchange network and its optimal operating condition (using mixed integer non-linear programming, Papalexandri et al., 1994).

This section reviews the different mathematical programming approaches that have found application in mass exchange network synthesis. This thesis is concerned with gradientbased methods because of their minimum dependence on random search techniques. The solutions that were obtained from stochastic optimisation methods (genetic algorithm and simulated annealing) will be compared where possible, with those obtained from this thesis.

A necessary differentiation in optimisation methods is between superstructure-based (Sbased) optimisation and superstructure-independent (SI) optimisation. Most SI methods are automation procedures for the pinch approach whilst the S-based methods do not consider the effect of the pinch.

\subsubsection{Linear programming}

El-Halwagi and Manousiouthakis (1990a) used linear programming (LP) to determine the minimum operating cost and locate the mass transfer pinch points of a mass exchange network. The authors assigned a minimum value for the composition difference $\varepsilon$ and generated a composition interval diagram consisting of a series of $n_{\text {int }}$ composition intervals which correspond to the supply or target compositions of each stream. Each interval is 
defined according to Figure2.9. Note that a similar interval was presented in Figure2.8 for targeting.

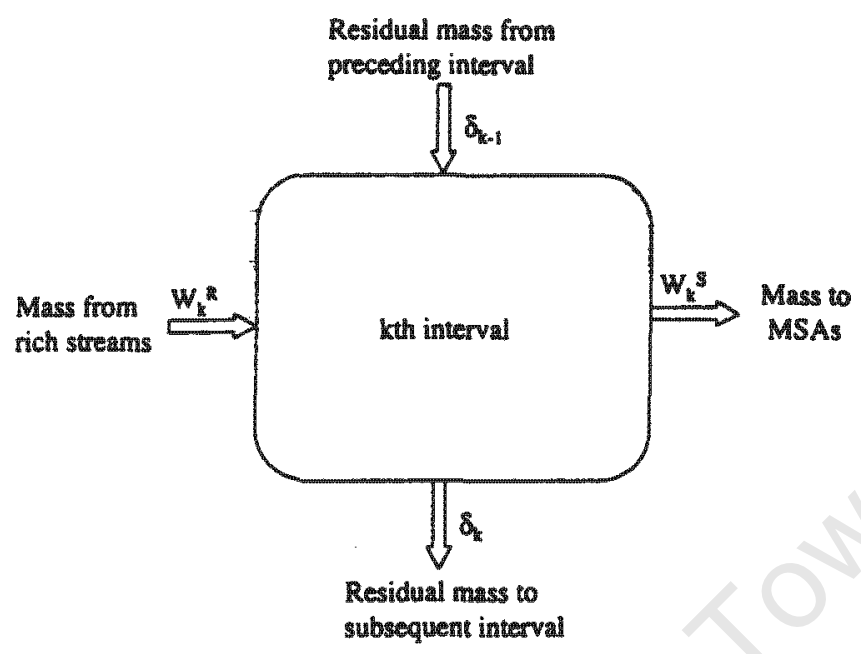

Figure 2.9: Mass balance around the $k^{\text {th }}$ composition interval

The LP model then includes

- successive overall material balance equations around each interval starting from the $k=1$ interval to the $k=n_{\text {int }}$ interval given by

$$
\delta_{k}-\delta_{k-1}+\sum_{j \in S_{k}} L_{j} \Delta x_{j, k}=G_{i}\left(y_{k}-y_{k+1}\right)
$$

- the constraints on the initial and final residual mass-exchange loads

$$
\delta_{0}=\delta_{n_{\text {int }}}=0
$$

- the objective function

$$
Z=\min \sum_{j \in S} c_{j} L_{j}
$$

where $c_{j}$ is the unit cost of the lean stream $j$.

The location of the pinch points between two consecutive intervals, $k$ and $k+1$, is indicated when the residual mass-exchange load $\delta_{k}$ vanishes. The solution to the LP problem will yield optimal values for all the lean stream flowrates, $L_{j}$. 


\subsubsection{Mixed integer linear programming}

In the second part of the LP work of (El-Halwagi and Manousiouthakis, 990a), a mixed integer linear program (MILP) transshipment problem is solved to identify the minimum number of mass exchange units compatible with the minimum-utility design. The transshipment model is similar to the ancient problem of determining the shortest distance travelled by a salesman. The rich streams are regarded as sources, the composition intervals as nodes, and the lean streams as destinations.

The MILP model uses pinch points determined from the LP solution to partition the synthesis problem into $n_{\text {pinch }}+1$ subnetworks whilst the lean stream flowrates are used to determine the upper bound on the mass exchanged between streams $i$ and $j$ in a particular subnetwork.

Each subnetwork is solved independently according to the objective of minimising the total number of exchangers in Equation2.17 by summing the binary variables $E_{i j m}$.

$$
Z=\min \sum_{m=1}^{n_{p}+1} \sum_{i \in R_{m}} \sum_{j \in S_{m}} E_{i j m}
$$

s.t

$$
\sum_{k \in S N_{m}} W_{i j k}-U_{i j m} E_{i j m} \leq 0 \quad i \in R_{m}, j \in S_{m}
$$

where

$$
U_{i j m}=\min \left\{\sum_{k \in S N_{m}} W_{i k}^{R}, \quad \sum_{k \in S N_{m}} W_{j k}^{S}\right\}
$$

In the MILP model, the constraint given by Equation2.18 ensures that mass $W_{i j k}$ is exchanged in an interval if and only if there is a match (which is denoted by a binary variable-value of 1 ) otherwise the constraint is not satisfied and then another match is considered. The formulation in Equations2.18and2.19 is called the big-M formulation because of the size of the feasibility parameter $U_{i j m}$. Note that the use of a symbol $\mathrm{M}$ or $\mathrm{U}$ is unimportant. What is important is that the value of $U_{i j m}$ is according to Equation2.19, the smallest of the mass that can be transfered from a rich stream to a lean stream $\left(W_{i k}^{R}\right)$ or that a lean stream has the capacity to accept from the rich stream $\left(W_{j k}^{S}\right)$.

The solution of the MILP model also informs whether or not stream splitting is required, 
and the composition over which a stream ought to be split. Combining the information for all the subnetworks produces the design for the entire network. The final design is selected from the solution vector of these networks generated from various pre-assigned minimum composition differences. Capital cost may be indirectly included in the MILP objective function by multiplying each of the $E_{i j m}$ by a weighting factor that represents the relative cost of the exchanger unit matching streams $i$ and $j$.

\subsubsection{Non-linear programming}

Lee and Park (1996) proposed a two step synthesis procedure that uses Process Graph Theory to generate and evaluate feasible MEN structures (Step 1) and then solves a nonlinear programming (NLP) model to obtain the operating conditions for each network generated in the first step (Step 2). Two structures are generated in step 1: a maximal structure and a solution structure. The maximal structure is a union of all feasible network structures that satisfies some process graph axioms. The solution structure contains the mapping and operating conditions that are optimised in the NLP model. Every feasible network is embedded in the maximal structure.

For each of the feasible networks, an NLP optimisation problem is generated and then solved. The optimum solution, featuring a minimum TAC is selected from the many solved NLP optimisation problems. The optimal network selected from the NLP is based on a fixed minimum composition difference, $\varepsilon$.

Benko et al. (2000) used a non-linear program for the optimal water allocation problem. Here a superstructure is generated and modelled as an NLP problem. The selection of the final flowsheet is determined by analysing the flowrates of streams entering and leaving a mass exchange operation as well as the amount of mass exchanged in a particular match. Those streams or units whose flowrates or capacities are approximately zero are dropped from further consideration in the superstructure.

In Benkoetal.'s work, the complications with the solution of many NLP problems still exist. It is worsened by the fact that during the stream or unit elimination procedure, there are no guidelines regarding which streams or units to remove first. The current author believes that the sequence of stream or unit elimination has economic implications as driving forces of subsequent matches will be affected by changes to the flowrate of particular streams. 
Wilson and Manousiouthakis (1998) investigated a minimum utility cost problem for a single mass exchanger handling multiple components. The NLP model was optimised subject to a bounded number of stages and lean stream flowrate. The determination of the number of stages at either bound (lower or upper) requires the use of logical operators to select the form of the Kremser equation (Equations2.10and2.11) subject to a value of the removal factor $A_{i j}$. It is not clear how the NLP algorithm was implemented for the selection of Equation2.10or2.11 for each bound.

The requirement for logical operators adds a complication in the use of mathematical programming methods that was manually dealt with in the insight-based pinch method. These complexities necessitate the use of computational tools that have an ability to handle logical operations. More than just generating flowsheet structures, mixed-integer non-linear programming, discussed next, can be used to model these logical operators.

\subsubsection{Mixed-integer non-linear programming}

The MINLP approach is based on three synthesis steps: (1) the generation of a superstructure that embeds the optimal flowsheet, (2) the description of the superstructure through the use of balance and sizing equations and (3) the generation of solutions to the model from which the optimum flowsheet is selected. Superstructures are graphical representations of the interconnection of streams and mass transfer units. The transshipment model discussed in Section2.3.2 is an example of a superstructure.

The developments in this thesis are based on mixed integer non-linear programming and thus more emphasis will be placed on the review of MINLP approaches in MENS. The discussion is based around the type of superstructure that is used to model the MENS problem. The superstructures that are identifiable in mass exchange network synthesis where MINLP has been applied are the transshipment model (El-Halwagi and Manousiouthakis, 990b), the state-space model (Bagajewiczetal.,1998) the hyperstructure model (Papalexandrietal.,1994) and the interval-based superstructure (Comeaux, 2000) discussed in Section2.4.3. Detailed mathematical descriptions are only given for the hyperstructure model since it forms the basis of this thesis. 


\section{Step 1: The superstructure representation}

\section{The transshipment model}

El-Halwagi and Manousiouthakis (1990b) presented an MINLP model based on the transshipment model superstructure (?) to solve MENS problems featuring incompatible multiple component problems. The transshipment superstructure regards the rich streams as sources and the lean streams as destinations whilst the shipped commodity is the desired species. The superstructure can, as shown before, be optimised as a linear programming problem with or without integers.

\section{The state-space model}

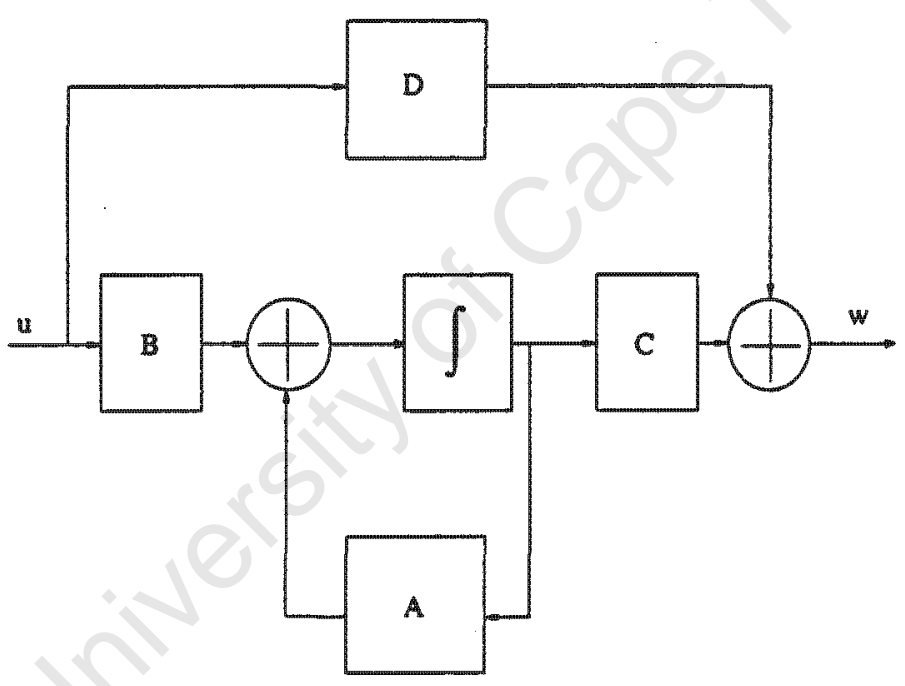

Figure 2.10: I-S-O representation of a dynamical system

Bagajewicz et al. (1998) presented a framework and application of the state-space approach for the synthesis of mass and heat exchanger networks (seeFigure2.10). The state space approach partitions a set of variables in a system into input $(u)$ and output $(w)$ variables. The sets over which the variables can assume their values are in turn referred to as the input and output spaces. The input-output relations (I-O) are such that the knowledge of the inputs and the I-O relations does not provide knowledge of the output. Any set of variables whose knowledge, when complemented with the knowledge of the system inputs and the I-O relations, uniquely determines the system outputs is referred to as the set of state variables. The set over which the state variables assume their values are referred 
to as state space and the relations that help establish output uniqueness based on state knowledge are referred to as input-state-output (I-S-O).

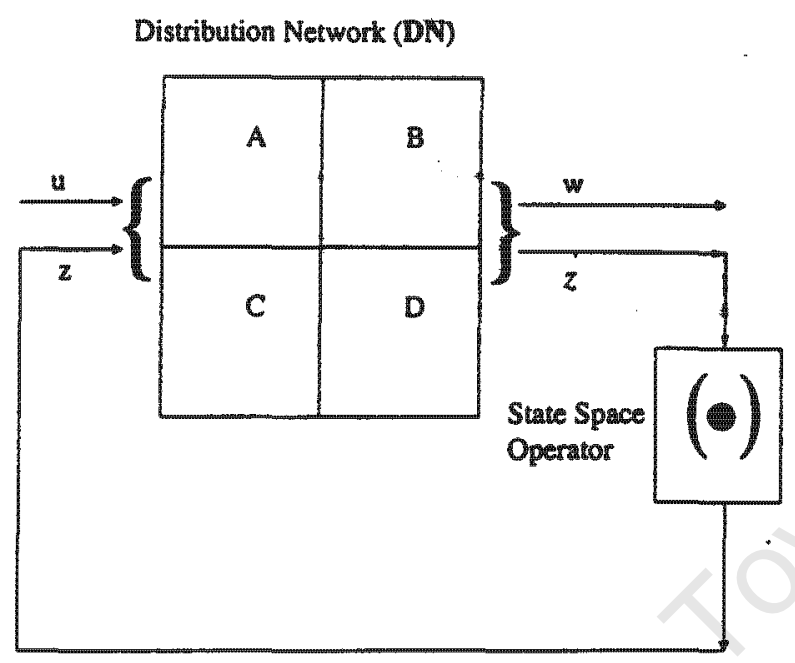

Figure 2.11: Alternative I-S-O representation of a dynamic system

The operators A, B, C and D in Figure2.10 are algebraic operators that can be represented in a matrix form $(D N)$ in Figure2.11 to process the input and the state information and then generate the output and the state derivative information. Thus the matrix $D N$ determines the process networks from input and output variables such as inlet and target compositions of streams. The state-space operator, $(\bullet)$, in Figure2.11 is said to be able to embed the following

1. a superstructure operator

- made by making many junctions within the distribution network to allow for multiple matches in the state operator. The motivation for many junctions is that the superstructure operator excludes stream bypass or splitting and a stream pair matches only once;

2. an assignment operator

- within the assignment operator, each stream is matched only once and multiple matching is allowed by including additional junctions in the $D N$ for each potential match. In the assignment operator, a small number of junctions for each stream is used at the start of the assignment and the junctions are increased until there is no longer a change in the solution; 
3. a pinch operator

- the pinch operator allows for the determination of minimum utilities. A concentration, mass-load diagram is employed to enforce the first and second laws of thermodynamics. This pinch operator may as well be for thermal pinch;

4. a split-matching operator, and

- this operator splits every rich stream entering the operator into as many streams as lean streams present.

5. a block partitioned operator(Zhu, 1995).

- the block partitioned operator consists of a mass pinch and a heat pinch block. The blocks may be connected in parallel or series. Block partitioning is useful in combined heat and mass transfer since the temperature level at which mass transfer should take place is optimised through the trade-off between the MSA and thermal utility costs.

In the assignment operator the optimal assignment of matches is achieved when the solution does not change with any further increment of the number of junctions. There are, however, no guidelines as to which streams should incur an additional junction first. The current author believes that in order to cover all possibilities it is required that the criterion to stop increasing the number of junctions should be subject to the consideration of all the alternatives or sequence of the junction-increments.

Whilst it is attractive in the utility operator to consider simultaneously the utility consumption required by each match, this has the potential of generating many small, complex flowsheets characterised by coolers and exchangers attached to major heat and mass exchange units that are aimed at utilising at best, process streams first before considering external utilities.

\section{The hyperstructure model}

Papalexandri et al. (1994) proposed an MINLP model for the simultaneous generation of a network and determination of the TAC without resorting to any pinch decomposition concepts. The match-network hyperstructure is described in Figure2.12 and involves the following features: 


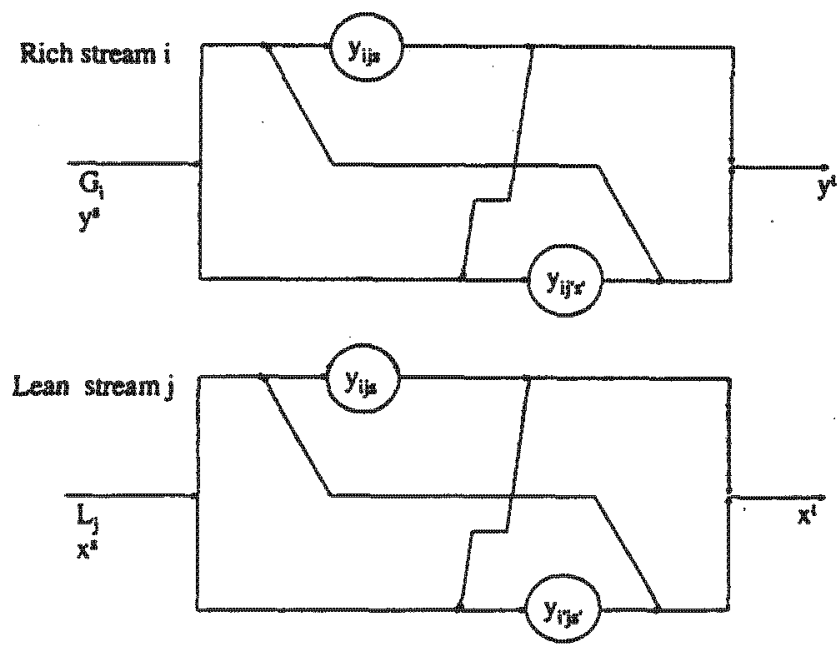

Figure 2.12: The hyperstructure for the rich and lean streams

- Each match between a rich and a lean stream corresponds to a potential mass exchange unit

- Each stream entering the network is split towards its potential mass exchange unit. This is different to the state-space representation where many junctions are constructed prior to the streams entering the superstructure operator.

- Unlike in pinch decomposition where the network is partitioned a priori into above and below pinch, in MINLP the partitioning into subnetworks allows for multiple mass exchange between two streams. It is not clear from this work what the relationship is between the model solution and the number of subnetworks. Too many subnetworks will increase the number of binary variables and make the problem difficult to solve.

- Prior to each mass exchanger there is a mixer where the flow from the initial splitter and the bypass flows from all the other potential exchangers of the stream are merged into a feed stream into the exchanger.

- Similarly, after an exchanger, a splitter is considered where the exchanger outlet stream can be driven to the final mixer or the other exchangers of the stream. Note this excludes the exchange from which the lean stream is coming. Thus self-recycle is forbidden in the hyperstructure.

- The integers in the hyperstructure represent the existence $\left(y_{i j s}=1\right)$ or absence 
$\left(y_{i j s}=0\right)$ of a match within a subnetwork and they determine the splitting and mixing policy of the network.

\section{Step 2: The mathematical description of the superstructure}

Only the mathematical description of the hyperstructure model is discussed in this section. For a detailed description of the hyperstructure model the reader is referred to Papalexandri et al. (1994) or AppendixC. The model consists of the following

- Overall mass balances at the initial splitter of each stream

- Overall mass balances at the mixer preceding each mass exchanger for each stream

- Overall mass balances at the splitter after each exchanger for each stream

- Mass balances for the transferable component at the mixer preceding each exchanger for each stream

- Mass balances for the transferable component at the final mixer of each stream

- Mass balances for the transferable component at each potential exchanger for each stream

- Feasibility constraints with a pre-specified value of the minimum composition difference, $\varepsilon_{i j}$ to ensure feasible mass transfer.

It is important that the minimum composition difference is set at a low enough value so that during optimisation the solution cannot be trapped at the lower bound of $\varepsilon_{i j}$. In this thesis an evaluation of the driving force for each match in the final network will be carried out .

Intuitively it would appear that in an optimisation problem there is no need to set a minimum value for $\varepsilon_{i j}$ as too small a value will lead to networks that require a large capital investment. However, most solution search algorithms depend of the existence of smooth functions in the model. The less non-linear form of the Kremser equation (Equation2.10) requires a lower bound on the composition difference to prevent the solution search from searching in regions where the equipment sizes are indeterminate. 
- Logical and connectivity constraints

- Sizing equations for the mass transfer units

Various mass exchangers can be sized according to any of the conventional sizing models: based on column height (Equation2.14) or number of trays (Equation2.9). The use of the advanced sizing models presented by Hallale (1998) and summarised in AppendixB is explored in this thesis.

- Bounds on the variables

The bounded variables in the hyperstructure model are the mass flowrate of each lean stream, its final composition and the final composition of the lean stream. The outlet compositions from each exchanger are not bounded. Note that this means that intermediate streams may exceed target compositions of their parent streams.

- Objective function

The objective function of the MINLP model is to minimise the total annualised cost and determine simultaneously the optimal network configuration, MSA consumption and equipment sizes and is of the form:

$$
\min T A C=\sum_{j \in L}\left(A C_{j}\right)\left(L_{j}\right)+\sum_{(i, j) \in M p, s}\left(A C_{s t}\right) f\left(N_{s t, i j s}\right)+\sum_{(i, j) \in M t, s}\left(A C_{h}\right) f\left(H_{i j s}\right)
$$

Step 3: Transfer of the hyperstructure model to an MINLP solver

The MINLP model in Papalexandri et al. (1994) was solved via a Generalised Benders Decomposition (GBD) algorithm. The GBD algorithm finds a solution by iterating between two subproblems: an NLP and an MILP subproblem. A comparison of the GBD algorithm and the Outer Approximation algorithm(?), used in this thesis, in terms of how the iterations differ is given in Section2.3.5.

\subsubsection{Computational issues}

Many algorithms have been developed to solve MINLP optimisation problems. This section only concerns the major algorithms used in solving large MINLP models. These are 
the Generalised Benders Decomposition (?) and the Outer Approximation algorithms(?). The OA algorithm has other derivatives that consider the use of equality relaxations(?) and penalty functions(?). Both algorithms involve the solution of a series of NLP primal subproblems where the integers are fixed and MILP master subproblems where the integers are predicted. The algorithms differ in the formulation of the master problem. The OA master problem is based on the accumulated linearisation of the constraints and the objective function whilst the GBD technique is based on the dualisation of the original problem and on accumulated Lagrangian functions parametric in the 0-1 variables.

In this thesis the OA algorithm for equality relaxation and augmented penalty(OA/ER/AP) is used. Its advantage over the GBD method and other methods is that it does not assume that the solved mathematical model is convex but does in fact investigate this. Under certain assumptions concerning the convexity of the non-linear functions, an equality constraint is relaxed to be an inequality. When this assumption does not hold, a slack variable is added to the inequality constraint and a large penalty is included in the objective function to try and minimise the value of the slack variable.

\section{MINLP commercial solvers}

DICOPT (DIscrete and Continuous OPTimiser) is a commercially available solver implemented in GAMS for solving mixed-integer non-linear programming problems that involve linear 0-1 variables and linear and non-linear continuous variables (GAMS, 1999). The program is based on the extensions of the $O A$ algorithm for equality relaxation and augmented penalty. The MINLP algorithm inside DICOPT solves a series of NLP and MIP sub-problems and DICOPT uses existing NLP and MILP optimisers to solve these subproblems. In the NLP primal problem the $0-1$ conditions are relaxed. If the solution to this problem yields an integer solution, the solution stops. Otherwise it continues iterating between the primal problem and the MILP master problem wherein the 1-0 variables are predicted.

The stopping criterion for this algorithm is related to the convexity of the mathematical model. Convex problems can be solved to global optimality thus, for these problems, the master problem also provides a lower bound on the objective function. In a minimisation problem, as in all cases in this thesis, the lower bound increases monotonically as iterations proceed due to the accumulation of linear approximations. The augmented penalty can be used to implement a stopping criterion when assumptions concerning convexity do not 
hold. The criterion is based on the heuristic: stop as soon as the NLP primal problem starts worsening.

A DICOPT characteristic that becomes relevant later in this thesis is its ability to handle integer variables. DICOPT can only handle binary variables instead of general integers. This is relevant when the number of stages in a staged vessel have to be integers. Methods of achieving the integer values in such cases could be to fix the reported number of stages to the lower or higher integer value and re-solving the model. Another way could be to re-solve the model with a selected lower minimum number of stages and add to this a series of binary values. It can be anticipated that this increase in the number of binary variables will make the MINLP model more difficult to solve.

In a recent review, Grossmann et al. (1999) discusses the algorithms and codes for solving different mathematical programming problems in the automated design, integration and operation of chemical processes. Highlighted below are only those codes that were used in conjunction with DICOPT and applied in this thesis.

\section{CONOPT}

CONOPT or its recent version of CONOPT2 is an NLP solution optimiser that is based on a generalised reduced gradient (GRG) algorithm and is used in conjunction with DICOPT to solve the NLP primal part of the MINLP. The GRG algorithm computes Jacobians of the constraints in the NLP model and separates the variables into basic and non-basic variables. A line search is carried out for the variables within the non-basic variable set in a pseudo-Newtonian process. As noted the individual steps are much more detailed in the actual implementation of CONOPT. CONOPT has a fast method for finding subsequent feasible solutions but requires at first that the designer provide a good initial solution.

\section{CPLEX}

CPLEX is an MILP solver which uses a branch and bound (BB) algorithm with cuts to solve a series of linear programming subproblems. The linear subproblems can be solved using a modified primal simplex algorithm. The advantage of using CPLEX is that several alternative algorithms can be used to solve the linear programs depending on the size of the problem. The GAMS/CPLEX User Notes specify that sometimes it might be necessary to experiment with the different algorithms - primal simplex for most problems, dual simplex 
for highly degenerate problems and the primal-dual logarithmic barrier algorithm for large and sparse problems.

Both these NLP (CONOPT or CONOPT2) and MILP (CPLEX) solvers are called by DICOPT to generate global optimal solutions for convex MINLP models and locally optimal solutions for concave ones.

\section{Modelling language}

There are two major modelling languages that are aimed at solving large and complex mathematical programming models and wherein the algorithms discussed can be implemented: GAMS(Brooke et al., 1988) and AMPL. GAMS, acronym for General Algebraic Modelling System was developed by the world bank to create and solve multi-sectoral economy wide models and large simulation and optimisation models in many industrial sectors. AMPL is a development from Bell Laboratories aimed to overcome the rigid solution output strategy of GAMS and also provide an environment for post-solution data manipulation. Both languages have similar syntax with AMPL keeping most of the features of GAMS thus allowing for an easy interchange of models.

The details of the search algorithm of the solver implemented in GAMS or AMPL are hidden from the modeller. In this case, the modeller need not worry about acquiring knowledge on how to manipulate and control complex algorithms but can focus on providing a good mathematical description of the problem at hand and on expanding the mathernatical programming applications to other types of problems in his or her field.

The important feature of modelling in GAMS or AMPL is that the mathematical description can be made compact through the use of sets and this makes the representation of large and complex models easy whilst also simplifying the implementation of changes made to the specifications of a problem. In this thesis, GAMS will be used as the modelling environment. This choice is not based on the superiority evaluation but merely on the observation that both modelling languages have a similar construction and can be interfaced with a large number of solvers of mixed-integer non-linear programming problems.

A limitation in GAMS that becomes relevant in this thesis is its ability to handle logical operators or conditional expressions when the argument within the operator is a function of a variable - an example of which is the dependence of the Kremser equation on the absorption factor variable, $A_{i j}$, in Equation2.9. Parameters are well handled as arguments to select, 
for example, desired entries from sets or an equation to be applied to a particular type of a match that is pre-specified. This limitation is not necessarily a GAMS related limitation only but concerns the ability of the commercial MINLP solvers to actually handle logical operators. Commands are available in GAMS to model these operators as will be shown in Chapter3. Work currently carried out at Carnegie Mellon (TurkayandGrossmann,1996 and Caballero,2001) employs disjunctive programming (?) to model MINLP problems with discontinuous functions. Solvers of disjunctive programming models are based on the use of logic and boolean functions and it can be anticipated that these solvers, currently not available commercially, will be able to handle the logical operators required to handle the use of the Kremser equation.

\subsubsection{Summary}

\section{Realistic trade-offs}

The simultaneous determination of multiple-objectives provided by an MINLP framework allows for realistic trade-offs to be analysed. These objectives are derivatives of many alternatives and choices which, with the use of pinch methods only, would not have been possible to enumerate in finite time. The multiple-objectives refer to the many variables whose optimal values contribute to the economic objective function that is minimised. Examples of the alternatives and choices have been shown in this section to be

- the optimum trade-off between the target composition or flowrate reduction of a lean stream when there is an excess of process MSA shown on a mass pinch plot

- the trade-off between the structure of a network and its cost

- the sensitivity of the synthesis objective to variations in process parameters.

The sensitivity analysis is simplified by the fact that in the MINLP framework, once set up, parameters can be changed if and when deemed necessary without having to re-formulate the model. This is indeed different to the pinch analysis method, where for every changed parameter, new targeting, a new interval or grid diagram needs to be constructed, an optimume needs to be determined, and an initial design is generated and evolved to a final flowsheet. Although there are automated pinch procedures, these have so far not accounted for the supertargeting concepts. Floudas and Ciric (1989) noted that the supertargeting 
objective in mathematical programming, leads to an MINLP model. Unlike the statespace approach, the hyperstructure approach is yet to use pinch insights to realise the mathematical equivalent of supertargets.

\section{Model tractability and parameter variation}

Most of the optimisation methods require the solution of many NLP problems before the final flowsheet can be obtained. Current optimisation solvers cannot be expected to just output an optimal solution from just any presented model. Various modifications in the model, some large and others small, may need to be made in order to obtain the final solution. These modifications enhance the tractability of the model. Two categories of model changes can now be identified: model tractability and parameter variation.

Model tractability concerns efforts to manipulate equations and variables so as to reduce and simplify the mathematical model. An example of this may be the reformulation of quotients into products to prevent divisions by zero during the solution search. Parameter variation is when for example a constant parameter is varied in a number of trials to see its effect on a studied variable. A good example is in supertarget where even though the minimum composition difference is fixed during the determination of capital and operating costs, to see its effect on the total annual cost, many variations of $\varepsilon_{i j}$ or $\Delta y_{\min }$ are made after-which the TAC can be expressed as a function of the minimum composition difference, hence the variation of $\varepsilon_{i j}$ or $\Delta y_{m i n}$.

\section{Challenges}

The MINLP framework shows promise in dealing with the many alternatives present during design but the approach is limited due to the following challenges:

- preparing a superstructure that must indeed incorporate the optimum flowsheet

- setting up an appropriate model that describes the superstructure As noted there is a trade-off between a detailed description and mathematical tractability in the choice of modelling equations

- selection of a solution algorithm that can handle the model details. There are two parallel challenges in this: good modelling and robust solvers. This thesis does not 
aim to design an MINLP solver, but to use available solvers to integrate pinch analysis advances with MINLP models. Thus, model formulation is chosen as the first challenge to integrate the pinch analysis concepts with those from the mathematical programming approach. The ultimate achievement in terms of the solution algorithm will therefore be an algorithm that shows how the modeller (relying on pinch guidelines) and the solver (relying on the relationship between the properties of its solution search and the description of the model) can be integrated

- choosing an appropriate modelling environment that can handle complex operators. Conditional operators are required to switch between two types of Kremser equations with each equation accounting for the different regions of the absorption factor. This is a complication whose solution is not well discussed in literature. Current efforts to incorporate discontinuities in a model include the work on Disjunctive programming algorithms at Carnegie Mellon which are yet to be commercialised.

- providing a feasible initial solution to the solver

\subsection{Hybrid synthesis-tools}

Grossmann and Daichendt (1996) give a review on the new trends in optimisation-based approaches to process synthesis. In the review, the question of how the optimisation and targeting approaches, and heuristics for that matter, can be combined "in such a way that on the one hand the integration is conceptually consistant and rigorous, and on the other hand it exploits the strengths of each approach" is highlighted as one of the important research challenges that remain to be solved. This section reviews some of the work done at integrating different synthesis models in the area of mass exchange network synthesis. Kravanja and Glavic (1997) integrated the mathematical programming approach with pinch analysis to pre-screen HEN superstructures. A match-dependent area targeting method is proposed first followed by an NLP optimisation for the selected structure. The area cost targeting is based on heat load distribution and is constructed from balanced composite curves for each pair of hot and cold streams and on an anticipation of the actual number of exchanger units. The number-of-units constraint prevents the generation of flowsheets that have many small exchangers. Like the mathematically-based pinch approaches, this combination method fixes the utilities before estimating the heat exchanger area through an NLP optimisation rather than using binary variables to generate a network. 
Within the area of mass exchanger network synthesis, an example of work towards the simultaneous integration of insight-based tools such as pinch analysis and mathematical programming is that of waste interception networks(El-Halwagi, 1997), integrated design of water systems (Alva-Argaez, 1999), the NLP approach in a water allocation problem(Benkoetal.,2000) and recently the NLP work based on an insight-based superstructure for the synthesis of MENs with minimum total cost(Comeaux, 2000). Also, the state-space approach (Bagajewiczetal.,1998) had pinch operators integrated into the mathematical model. From each approach that is discussed in this section, the current author aims to elucidate the philosophy behind the integration of pinch analysis and mathematical programming.

\subsubsection{Waste Interception Networks}

Waste interception networks, unlike mass exchange networks, attempt to remove or minimise waste prior to it reaching the end of the pipe treatment units. The flow of waste within a process is tracked and intercepted using developed waste interception technology (ElHalwagi, 1997). The integration of pinch insights with the mathematical model is realised through the parameterisation of a rich composite curve, in a pinch diagram, as a function of path-diagram compositions. The path diagram represents the load of the targeted species through out the process as a function of its composition in carrying streams. The problem is solved as an MINLP optimisation problem with the objective of determining the optimum locations of intercepting pollutants, the extent of separation, and the separating agents to carry out the interception tasks.

Although the pinch-path diagrams provide the necessary integration of design tools, the total annualised cost is minimised by iteratively trading off the fixed cost and the operating cost using $\varepsilon_{i j}$. The application of WIN requires a process flowsheet to be already existing in order to track the path of a component in the process.

\subsubsection{Integrated design of waste water networks}

Alva-Argaez (1999) discussed the combination of water-pinch concepts with mathematical programming. The method is based on a superstructure model formulated as an MINLP problem. In the superstructure, existing units are determined by the number of units in the network. Binary variables merely account for the existence or non-existence of connections 
between the sources and sinks. The superstructure provides for options for the lean (water) streams to merge. The number of processing units are known a priori together with the mass flowrate of the process streams through the units. Subject to the values of the binary variables, a connection can be eliminated. Insights from pinch analysis are integrated in the synthesis problem through the solution strategy based on the pinch insight that: in all water-using operations, at least one of the contaminants will reach its maximum permissible value in the optimal solution.

$$
L_{\min }=\max \frac{\text { mass load }}{c^{\text {out }, \text { max }}-c^{i n, \max }}
$$

The limiting water flowrate (Wang and Smith, 1994) is described by an inverse relationship with the outlet concentrations of the contaminants, according to Equation2.20. The mass load and the inlet concentration, $c^{i n}$ are fixed parameters, thus the outlet concentration $c^{\text {out }}$ the must be maximised to reduce the water flowrate. This maximum concentration is reached by limiting contaminants and a penalty term is included in the objective function for the excess water that will be required to remove those contaminants whose outlet compositions would not have reached their maximum values. The networks in this work were generated using a combination of NLP and water-pinch analysis.

This work tackled what is to be an important aspect of this thesis: a method of selecting an appropriate Kremser equation subject to a value of the absorption factor $A_{i j}$. Alva-Argaez modified the Kremser equation to be independent of the solute concentration and thus only the flowrate of water is a changing parameter in the Kremser equation. The independence of the Kremser equation from the concentration is achieved by placing the constraint on the concentration of the species leaving a mass exchanger, that it will be at its maximum if a minimum fresh water consumption objective is to be realised. This maximum is given in the problem specification, thus in the projected space, the concentration is fixed. Once the concentration is fixed, a mixed-integer linear program for the determination of flow patterns in a water network is solved. These flow patterns are then used in a linear program, where a vector of outlet concentrations to be used in the next iteration is generated. The actual number of stages in an exchanger is simultaneously determined with the generation of the flow patterns in the MILP part, thus the resulting number of stages model is a linearised univariate function (Equation2.21) of the total water flowrate through a mass exchange unit. This method excludes the need for logical operators. The synthesis problem however, 
requires the solution of many MILP-NLP subproblems. In general mass exchange network synthesis, the flowrate of process rich streams or the mass-loads for the exchangers are not known ahead of design, thus the need for logical operators cannot be ignored.

$$
A_{i j}=f\left(L_{j}\right)
$$

\subsubsection{Insight-based superstructure}

Comeaux (2000) proposed an insight-based reducible superstructure(Figure2.13) to solve for mass exchange networks featuring minimum total costs. Comeaux uses the vertical mass transfer concept to define the superstructure. The $y-y^{*}$ grid diagram, with the potential stream matches in each interval is described as an NLP model. Unlike the pinch grid diagram, the reducible superstructure does not use the minimum approach composition to partition the flowsheet. The minimum composition difference is considered an optimisable parameter. In addition, each lean stream in an interval has a by-pass stream (le) allocated to it.

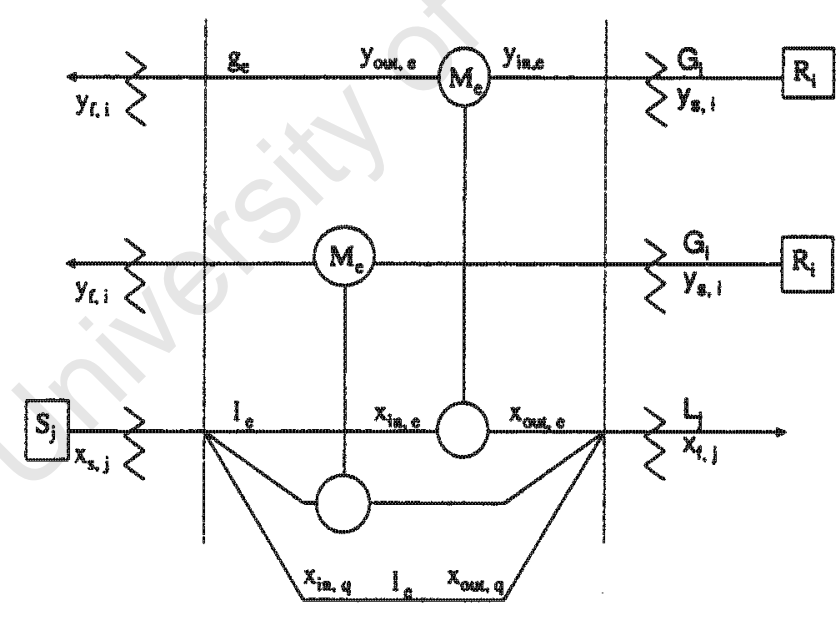

Figure 2.13: A typical interval in a reducible superstructure

The superstructure was shown to reduce for example, for a problem with 4 streams, from a maximum of 54 matches to just about 8 matches. This illustrates the advantage of the insight-based superstructure to reduce the dimensionality of alternatives that have to be considered in optimisation-based approaches. The superstructure is, however, limited to parallel flow configurations and capital cost models that are continuous. 
Lean streams entering the interval are already split to exchange mass once with a rich stream. This exclusion would work well when the composition range $\Delta x_{j}^{k}$, within an interval is small. Consider a stream that, when placed in the superstructure, has some part of it crossing through a wide composition interval. Let that part of the stream be called a sub-stream $j^{\prime}$ with a variable inlet and outlet interval composition according to Equation2.22.

$$
x_{j}^{s}-x_{j^{\prime}}^{i k} \leq \Delta x_{j^{\prime}}^{k} \leq x_{j}^{k}-x_{j^{\prime}}^{o k}
$$

In the case where the size of $\Delta x_{j^{\prime}}^{k}$ becomes large as a result of either $x_{j^{\prime}}^{i k}$ turning towards $x_{j}^{s}$ or $x_{j^{\prime}}^{\text {ok }}$ turning towards $x_{j}^{t}$ the sub-stream attains the inlet and outlet composition of the parent stream. Both series and parallel connections for this stream with the rich streams should now be explored for the best trade-off in exchanger size and MSA consumption. The reason why it would be possible to get near-optimal solutions even if certain matching configurations are not considered is that in most MENS problems the streams are dilute and require a small change in composition.

Capital cost models vary in complexity. Some models contain fixed cost and are discontinuous. These discontinuities can be overcome or modelled through the use of binary variables. The Comeaux model, like that of Benkoetal,2000, is NLP-optimised and thus limited to continuous sizing and cost functions.

\subsubsection{Hybrid tools: Concluding remarks}

\section{Model extraction}

It is clear that the limitations of mathematical programming are carried through to the hybrid method. Models are difficult to extract because of modelling strategies or methods that are not so well explained. The ability to successfully achieve a mathematical model that is tractable and yet descriptive enough to account for important logical and physical features is a necessity.

\section{Sequential solution algorithms}

Like the mainstream mathematical approaches the utilities are fixed before the network is generated. Where the utilities are allowed to be simultaneously determined, the structure 
is generated from the analysis of flow and load variables. The complexity of the structure is not incorporated in the trade-off between exchanger size and MSA consumption. In fact, generating structures and subsequently determining their capital cost, from analysing the flow and load variables as continuous variables is not really trading-off.

\section{Early account for process interactions}

The hybrid approach ensures that there is some level of involvement by the design engineer in the synthesis stages. In the hybrid strategies discussed this involvement spans from generating the superstructure to selecting the best solution from a vector of solutions. Although the engineer is unable to evolve a network as in pinch analysis, he or she has has the pinch method to provide near-optimal solutions and then the mathematical framework to generate better solutions. The problem, however, is that once a near-optimal solution is obtained, the computational effort for a better solution cannot be justified at the early stages of a project where even the thermodynamic parameters and sizing models are still estimates. Despite the lack of accurate data there is still a need for an MINLP framework to be available at an early stage because once the framework is made available, changes that are made to the model or new design information can be easily implemented without the strict reliance on the graphical pinch method.

\subsection{Thesis Contributions}

Three main contributions are anticipated in this thesis. Firstly, the supertarget work of Hallale(1998) has shown promising results when compared with that of El-Halwagi and Manousiouthakis (1989, 1990a, 1990b), El-Halwagi and Srinivas (1992) and Papalexandri et al. (1994). With the targets determinable ahead of design, the hyperstructure model is re-worked so that the pinch-based targets can be used as a yard-stick against which the quality of the MINLP solution can be determined. The decisions that the designer is to make once an MINLP solution is observed to be poor involve consideration of the pinch-based driving force diagrams, initialisation of MINLP models and the relaxation of feasibility constraints.

Mass exchange networks that are generated by using the supertargeting method are economically better and structurally simpler than those reported by MINLP methods (seeHallale,1998). The network design itself however, can only be achieved once the targets have 
been set. This sequential nature of the pinch design method does not simultaneously take into account the network's structure and total cost. The MINLP framework can. Thus, the targets from pinch will provide the much needed bound for the MINLP model while the simultaneous optimisation ability of the MINLP framework will generate the trade-offs for pinch analysis to meet the additional requirement of considering the interactions between the flowsheet structure and the continuous variables in the model.

Secondly, the application of the hybrid tool is carried out on an industrial case study. In the synthesis problem, although the MSAs are selected, the generation of appropriate equilibrium functions becomes a necessity. A reactive mass exchange operation is considered here and the generation of an equilibrium function is integrated into the MENS procedure. Although this is a topic previously discussed within the area of chemistry research, its inclusion into MENS was superficial during the work of El-Halwagi and Srinivas (1992) and Srinivas and El-Halwagi (1994).

The third contribution is based on the identification of the limitations in MINLP modelling that must be overcome in order to achieve feasible and optimal solutions. Logical operators are proposed for the selection of an appropriate form of the Kremser Equation (see Equations2.10and2.11) and an approximation of the McCabe-Thiele construction is proposed to analytically determine the number of stages in an exchanger that features a non-linear equilibrium function.

All these contributions attempt to meet some of the challenges that were said to be remaining in process synthesis (seeSection1.5) - the integration of pinch insights with an optimisation approach, the reduction of the combinatorial search and the handling of complex models. 


\section{Chapter 3}

\section{Sizing models}

The mere formulation of a problem is far more often essential that its solution, which may merely be a matter of mathematical or experimental skill

Albert Einstein (1879-1955)

The first part of this chapter explores and proposes various logical operators to be included in the hybrid model in order to account for the two forms of the Kremser Equation (Equations2.10and2.11) subject to the value of the absorption factor A. The second part of the chapter explores the problem of sizing staged vessels when the network contains streams with non-linear equilibrium functions. An approximation of the McCabe-Thiele stepping off process is proposed for inclusion in the hybrid model. The approximation is based on the average composition difference between matching streams.

\subsection{Logical Operators}

Optimisation methods are hampered by the availability of modelling tools that can handle the use of boolean, logical or conditional operators. Logical operators are special expressions that evaluate to a value of True or False. Numerical expressions can also serve as logical conditions by following the convention that the result of a relational operation is zero if the assertion is false, and one if it is true. Within the mass exchange network synthesis approach, staged vessels sized through the Kremser equation require that the selected model handle the sizing process based on two different forms of the Kremser equation subject to the value of the absorption factor $\mathrm{A}$. 
While the work of Alva-Argaez (1999) attempted to deal with the problem of the Kremser equation by formulating the number of stages as a univariate function of the flowrate or assuming that the absorption factor is fixed to some optimal value. It is however, still not clear from any of the optimisation work of Papalexandri et al. (1994), Benko et al. (2000) and Comeaux (2000) how the logical or conditional operators for the selection of the Kremser equation was implemented. As optimisation research continues, conditional operators need to be included in the development of the modelling environments and solvers. This section outlines the strategies that can be used to model logical operators for the selection of the appropriate Kremser equation using current solvers.

\section{GAMS conditional expressions, assignments and equations}

GAMS implements logical operators through the use of a built in operation: the dollar (\$) operator. The condition that controls the $\$$ operator must be fixed variable or a parameter. That is, for the appropriate Kremser equation to be selected, the absorption must be known ahead of the optimisation or after the solver has found a solution. Clearly this is inappropriate as the absorption factor changes with the many other variables in the model during optimisation. The \$ operator can however be successfully used in a multi-solve loop fashion.

First, solve the MINLP model using Equation2.10 and include the $\$$ operators that if in the reported solution, there is an exchanger whose value for the absorption factor is 1 , then an alternative Kremser equation should be used. Next fix the $A=1$ values and re-solve the model this time sizing the fixed-A exchangers according to Equation2.11. This algorithm is now summarised.

1. Define the true condition for the standard Kremser equation

$$
K r_{i j}\left(\$ \text { condition }_{i j}\right) \quad N_{s t, i j}^{\mathrm{i}}=N s t\left(A_{i j} \neq 1\right)
$$

2. Define the false condition for the standard Kremser equation

$$
K r_{i j}\left(\$ n o t \text { condition }_{i j}\right) \quad N_{s t, i j}^{2}=N s t\left(A_{i j}=1\right)
$$

3. Solve the MINLP model for the true condition part 
4. Implement an if controller for matches whose level values for the absorption factors is 1

$$
\text { If } A_{i j} \cdot l=1, \text { condition } n_{i j}=0 \text { and } A_{i j} \cdot f x=1
$$

5. Re-solve the model with the new constraints

The liraitation of this approach is that during the optimisation of step 5, those exchangers that did not have absorption factors of unity may take on values of unity because the weighting on the number of stages is now different. It is becoming evident that one can end up fixing all the absorption factors to 1 thus returning to the original limitation that this approach was hoping to overcome. The only difference in the case of the multi-solve approach, when the absorption factor ends up being fixed to unity, is that the number of stages are reported by Equation2.11 and not by Equation2.10 which will be indeterminate due to a division by zero.

\section{Summation of $N_{s t}$}

The summation model is based on the observation that either of the Kremser equations can dominate the exchanger sizing and the optimisation process has to select a combination of these Equations that will give the minimum exchanger size. Should all the exchangers have an absorption factor of 1 , then the second term in Equation3.1 automatically determines the final number of stages.

$$
N_{s t}=\frac{\ln Y r_{i j}}{\ln A_{i j}}+\frac{y_{i, \text { in }}-y_{i, \text { out }}}{y_{i, \text { out }}-m_{j} x_{j, \text { in }}-b_{j}}
$$

For all other values of the absorption factor, the summation model will be an overestimate due to the number of stage contribution of the second term.

\section{Quadratic constraint}

Szitkai et al. (2001) modelled the logical operation using a binary variable whose value of 1 or 0 was determined by the result of a quadratic equality(Equation3.3). The binary variable is then implemented in Equation3.1 according to Equation3.2. The result of this formulation is that when the absorption factor is unity, the binary variable takes the value of zero and the number of stages is determined by Equation2.11. For absorption factors 
other than unity, the binary variable takes the value of unity while $\beta_{i j}$ takes on values that satisfies Equation3.3. The number of stages is then determined by Equation2.10.

$$
N_{s t, i j}=Y_{i j}^{k} \frac{\ln Y r_{i j}}{\ln A_{i j}}+\left(1-Y_{i j}^{k}\right) \frac{y_{i, \text { in }}-y_{i, \text { out }}}{y_{i, \text { out }}-m_{j} x_{j, \text { in }}-b_{j}}
$$

and

$$
\left(A_{i j}-1\right)^{2}=\beta_{i j} . Y_{i j}^{k}
$$

whereby

$$
0.01 \leq \beta_{i j} \leq 9800
$$

The quadratic constraint can be used without the boundary variable to totally avoid the determination of the number of stages at $\mathrm{A}=1$ by constraining $A_{i j}$ according to Equation3.4.

$$
\left(A_{i j}-1\right)^{2} \geq \beta^{2}
$$

whereby $\beta=0.001$, and

$$
\begin{aligned}
& A_{i j} \geq 1.001 \\
& A_{i j} \leq 0.999
\end{aligned}
$$

Numerical problems are experienced regarding the scaling of Equation3.4 when smaller values of $\beta$ are used. Larger values exclude a wide range of near unity $A_{i j}$ values from the model and in the process eliminate what could possibly be optimal solutions.

Szitkai et al. (2001) showed a comparison of the binary logic with other formulations such as the convex hull, bigM and multi-M. In this work the solution time of the binary logic was shown to be faster than the other formulations. The fast solution time was attributed to the lesser number of binary variables that are used in the binary logic as compared to the other formulations. 


\section{Binary approximation}

Based on the learning from the formulation of the quadratic constraint, the binary variable can be approximated as a continuous variable thus reducing the number of binary variables to be considered in the model as previously observed in the formulations of Szitkai and co-workers. The binary approximation assumes that $Y_{i j}^{k}$ is a continuous variable which can take the value of zero or close to 1 . The variable $\beta_{i j}$ is then explicitly modelled as a function of $A_{i j}$. Equation3.3 is then reformulated as

$$
\left(A_{i j}-1\right)^{2}=\left(A_{i j}-1+0.001\right)^{2} Y_{i j}^{k k}
$$

where $Y_{i j}^{k k}$ is the binary approximation variable also replacing $Y_{i j}^{k}$ in Equation3.2.

The result is that when the absorption factor is 1 , the number of stages is determined as in the binary based quadratic constraint. For all other absorption factors however, the number number of stages is approximated by a strong contribution of Equation2.10 and a significantly reduced contribution of Equation2.11 as $Y_{i j}^{k k}$ approaches 1.

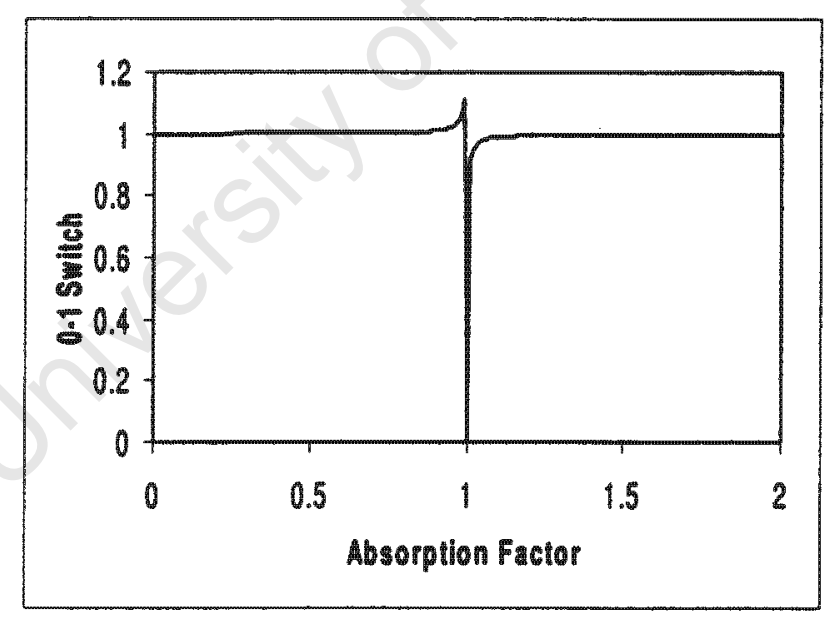

Figure 3.1: The logical operator based on the binary variable approximation

The logic operation given by Equation3.5 can be implemented in an NLP framework to address the Kremser difficulties. Figure3.1 illustrates the behaviour of the logical operator in switching between the two forms of the Kremser equation and thus showing that it can be used with confidence in an NLP model or to eliminate additional binary variables in an MINLP model. 


\section{Driving force based $A_{i j s}$}

Driving force based absorption factors use the $y$-y* composite curve to determine the range of values of $A_{i j s}$ that can ensure that the design targets are met. The hypothesis made here is that when the hybrid model is constrained according to the values of $A_{i j s}$ obtained from a $y-y^{*}$ composite curve, the resulting network or objective function will not only use optimal $A_{i j s}$ values but will also follow the TAC targets closely.

Recall that the absorption factor is a ratio of the match operating line and the slope of the equilibrium function. The slope of the equilibrium function is the constant parameter $m_{j}$, thus $A_{i j s}$ varies according to the ratio of the flowrates of the streams in a match. If this ratio, as reported by an MINLP solution, is similar to that obtainable from the composite curve, then the resulting $A_{i j s}$ would be optimal.

Note that the evaluation of whether a match makes the best use of driving force requires the operating line of a match to lie as close to the composite operating line as possible. In the determination of $A_{i j s}$ values, the requirement is that only the ratio of the slopes be similar.

The ratios can be predicted without an investigation of the $y-y^{*}$ curve by just using total stream instead of through-match flowrates. The advantage of using the composite curves is that the flowrates are already determined as best as possible and the help given by the curves is in locating the composition regions within which certain stream pairs may exist.

A summary of the results for these different methods for modelling logical operators is given in Table3.1. The methods were compared on a PII Celeron $533 \mathrm{MHz}$ PC employing CONOPT2 for the NLP subproblem and CPLEX for the MIP subproblem. The summation operator over-calculates the number of stages in the model largely because the contribution made by the $A=1$ part of Equation3.1. The quadratic and binary approximation operators reported the same total annual cost with different distribution in terms of operating and capital cost (as given by the number of stages). The large solution time observed from the fixed single-A operator can be attributed to the NLP solver attempting to search for variable values that satisfy the specified value of $A$. 
Table 3.1: Comparison of the logical operators to use with the Kremser equation

\begin{tabular}{|c|c|c|c|c|c|}
\hline Operator & $\begin{array}{c}\text { TAC } \\
\left(10^{3} \$\right)\end{array}$ & $\begin{array}{c}\text { Number of } \\
\text { stages }\end{array}$ & $\begin{array}{c}\text { Operating } \\
\text { cost }\left(10^{3} \$\right)\end{array}$ & $\begin{array}{c}\text { Number of } \\
\text { iterations }\end{array}$ & $\begin{array}{c}\text { Time } \\
(\text { seconds })\end{array}$ \\
\hline \hline Conditional & 265 & 30 & 25.6 & 4 & 1.32 \\
\hline Summation & 299 & 211 & 155.3 & 3 & 3.72 \\
\hline Quadratic & 226 & 49 & 156.5 & 3 & 3.5 \\
\hline Binary Approximation & 226 & 44 & 165.1 & 6 & 4.3 \\
\hline Drive force-based & 244 & 38 & 197.2 & 4 & 2.36 \\
\hline Fixed single-A & 263 & 77 & 187.8 & 8 & 5.14 \\
\hline
\end{tabular}

\subsection{Approximating ideal stages}

This section proposes an approximation method that can be used to analytically determine the number of stages of an exchanger when the equilibrium function describing the mass transfer is non-linear. The derivation of the Kremser equation is based on the assumption that the equilibrium function of the contacting streams is linear(Treybal, 1981). When non-linear functions are encountered, this assumption becomes invalid and an alternative analytical expression should be developed.

Ad hoc methods of sizing exchangers that feature non-linear equilibrium functions include the partitioning of a non-linear curve into linear segments and summing the number of stages from each segment. The implementation of linear segments in an optimisation procedure requires the prediction of compositions inside a transfer unit and thus it is a method which will work well when the mass exchange can be graphically expressed for example through the McCabe-Thiele diagram.

\subsubsection{Approximation philosophy}

The approximation method (McCabe-Thiele Approximation or MTA) that is proposed in this section is based on the definition of a step in the construction of the McCabeThiele diagram. A step consists of two parts: a horizontal line starting from the operating line to the equilibrium curve, and a vertical line starting from where the horizontal line intersects the equilibrium curve to the operating line. This procedure starts from the inlet composition of the lean stream, and ends when the horizontal line crosses the outlet composition of the lean stream. The approximation therefore assumes that the number of stages is given by the ratio of the composition-difference to the average driving force of an 
exchanger. Recall that the driving force is the difference between an operating line and the equilibrium curve on a McCabe-Thiele diagram. The average driving force $A D F$ is the average of the driving forces at the lean and rich end of an exchanger when the equilibrium line is linear.

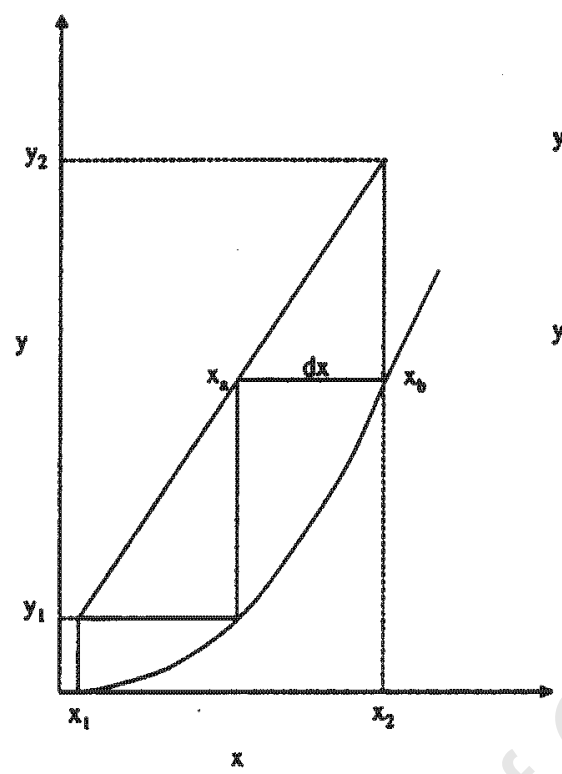

(a)

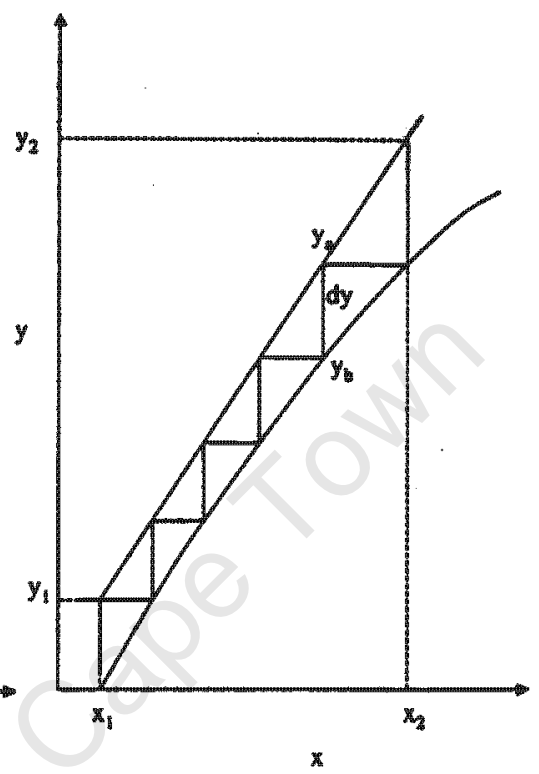

(b)

Figure 3.2: The use of the McCabe-Thiele method for non-linear equilibria.

(a) convex equilibrium function

(b) concave equilibrium function

To illustrate this development consider the McCabe-Thiele diagram in Figure3.2. In Figure3.2(a) the number of stages is determined by number of average lengths $d x$ determined at $y_{1}$ and $y_{2}$ that can be exist between $x_{1}$ and $x_{2}$. Similarly, for the exchanger described by Figure3.2(b), the number of stages is equivalent to the number of the average lengths $d y$ as determined at $x_{1}$ and $x_{2}$ that can fit between $y_{1}$ and $y_{2}$.

$$
\begin{aligned}
N_{s t m a, i j s} & =\frac{y_{i j s}^{I}-y_{i j s}^{O}}{A D F_{i j s}} \\
A D F_{i j s} & =\frac{1}{x_{i j s}^{O}-x_{i j s}^{I}} \int_{x_{i j s}^{i}}^{x_{i j s}^{O}} \Delta y(x) d x \\
\Delta y(x) & =y(x)-y^{*}(x)
\end{aligned}
$$


Equations3.6to3.8 are used to model the McCabe-Thiele approximation. $N_{s t m a, i j s}$ can be expressed in terms of the lean stream compositions (Equations3.9to3.11) by adopting the nomenclature of Figure3.2(a).

$$
\begin{aligned}
N_{s t m a, i j s} & =\frac{x_{i j s}^{O}-x_{i j s}^{I}}{A D F_{i j s}} \\
A D F_{i j s} & =\frac{1}{y_{i j s}^{I}-y_{i j s}^{O}} \int_{y_{i j s}^{O}}^{y_{j s}^{l}} \Delta x(y) d y \\
\Delta x(y) & =x(y)-x^{*}(y)
\end{aligned}
$$

The exact form of the integral of $A D F(x)$ or $A D F(y)$ will be determined by the type of the equilibrium function and it is the integrated form that is implemented in the hybrid model. AppendixB lists the expanded form of the most common types of non-linear equilibrium curves: the power (or polynomial) and exponential functions.

\subsubsection{Approximation accuracy}

To test the reliability of the MTA method, in Figure3.3 the number of stages determined from the Kremser equation for a number of matches featuring linear equilibrium functions are compared with those obtained from the MTA method. It can be observed that although the MTA ( Nstma) trend is similar to that of $N s t$ for the different values of $A$, the MTA method under-estimates the number of stages.

The matches that are compared in Figure3.3 were extracted from the solutions reported by Hallale(1998) and an example of such a match and test is illustrated here.

Consider a mass exchange operation featuring a linear equilibrium function of the form $y^{*}(x)=1.53 x$ and the following results for the composition and flowrates of the streams

\begin{tabular}{|c|c|c|c|c|c|}
\hline xin & xout & yin & yout & Le & $G e$ \\
\hline \hline 0.016 & 0.03 & 0.05 & 0.0347 & 2.22 & 2 \\
\hline
\end{tabular}

The operating line $y(x)$ is

$$
y(x)=\frac{L e}{G e}\left(x-x_{i n}\right)+y_{\text {out }}
$$




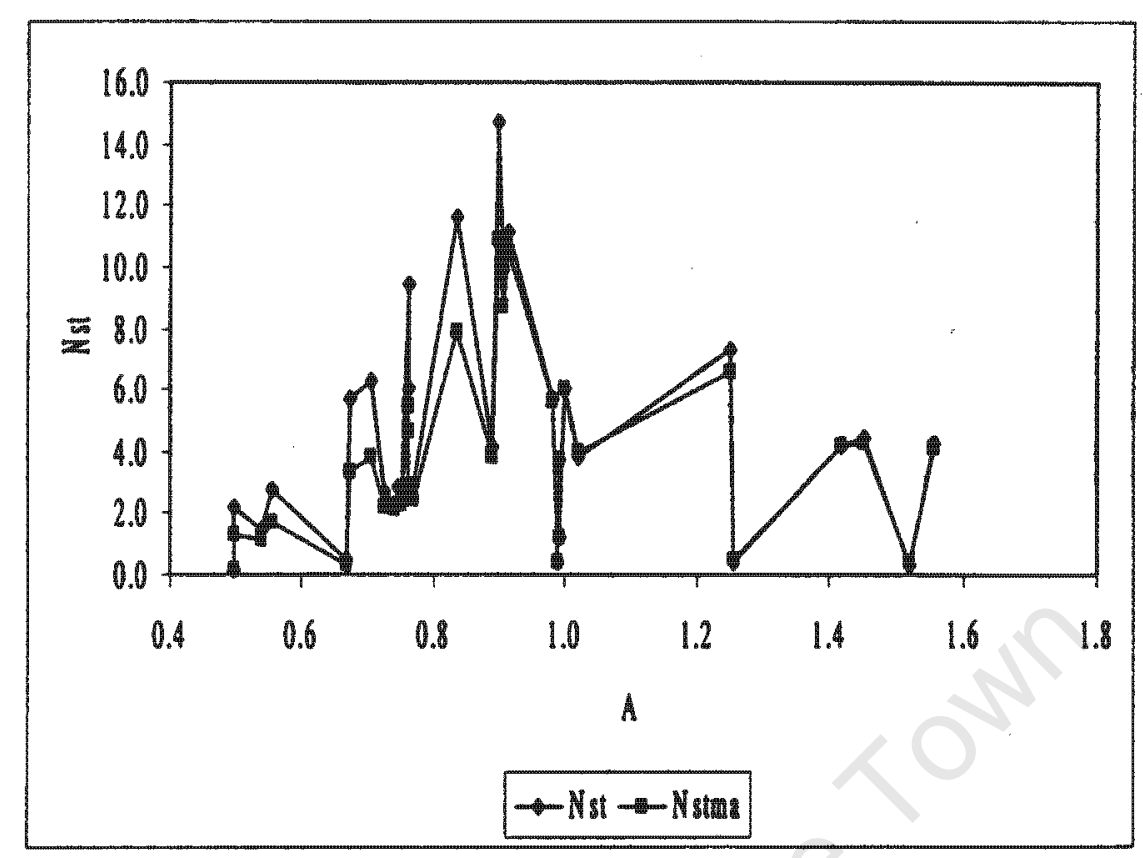

Figure 3.3: Comparison of the number of stages from the Kremser equation and from the MTA method

$$
=1.093 x+0.0172
$$

The average driving force, based on the rich stream is 0.0058 .

$$
\begin{aligned}
A D F & =\frac{\int_{0.016}^{0.03}(1.093 x+0.0172-1.53 x) d x}{0.03-0.016} \\
& =0.0058
\end{aligned}
$$

From Equation 3.10 the number of stages is 2.64, which when rounded off to the next highest integer is 3. The summary of results using the Kremser equation, the McCabeThiele construction and the Approximation method are given in Table3.2. The Kremser and the McCabe-Thiele method gives similar results as expected. 
Table 3.2: Number of stages determined by different methods

\begin{tabular}{|c|c|}
\hline Calculation method & Number of stages \\
\hline \hline Kremser & 2.75 \\
\hline McCabe-Thiele & 2.79 \\
\hline$A D F_{y}$ & 2.64 \\
\hline$A D F_{x}$ & 2.998 \\
\hline$A D F$ Arithmetic mean & 2.82 \\
\hline$A D F$ Geometric mean & 2.81 \\
\hline
\end{tabular}

\subsection{Summary}

In this section the problem of modelling logical operators for the successful optimisation of mass exchange network synthesis problems was illustrated. The proposed models can be classified as forcing models (summation $N_{s t i j s}$, binary quadratic, the conditional operator and the operating-line based $A_{i j s}$ ) and approximating models (the binary approximation and the quadratic-constraint). A true logical operation will be realised when the selection of the Kremser equation to use when a particular value of the absorption factor is obtained can be explicitly coded into the model to be executed as part of the optimisation process. What is important to note is that the choice of which model to use depends on the problem at hand and the level of detail or accuracy desired for the solution.

The author notes an alternative option could be the use of an alternative modelling environment which can explicitly handle conditional operators for variables. MATLAB has an excellent conditional handling facility that can be integrated within the GAMS environment. An important consideration in the integration is that the logical operation to be executed by MATLAB must be implemented within the GAMS solution search process and not after. If this is not the case, and the logical operation is done after a GAMS solution is generated, then the limitations of this process will be the same as those of the multi-solve \$technique except that this time the \$operation is called by an external modelling language.

Also in this section, a rapid and satisfactory method for the approximation of the number of equilibrium stages in a stage-wise exchanger was proposed. The method is based on insights gained from the definition of a step during the construction of the McCabe-Thiele diagram. The method is general since it can handle any form of an equilibrium function: polynomial, power, exponential or logarithmic types of curves. When the number of stages from the approximation method are compared to those from the Kremser equation (for the 
case of linear equilibrium line) the McCabe-Thiele Approximation fares comparably well. It is now possible to optimise and analytically determine the number of stages for stagedtype mass transfer units that have non-linear equilibrium functions. 


\section{Chapter 4}

\section{Hybrid Synthesis Method}

Anyone can vary the experiment in any number of ways. He that hits in this fashion on something novel and of use will have fame... The fame will be the product of luck and industry. It will not be the product of special talent.

Hilarie Belloc (1870-1953)

\subsection{Introduction}

This chapter explores the opportunities that arise from combining mixed-integer non-linear programming with insights from pinch analysis for the synthesis of optimal mass exchange networks. A successful integration will be realised if the method is conceptually consistant, rigorous and exposes areas of improvement on the designs that are based on a single synthesis technique. The optimisation approach is based on the MINLP framework of Papalexandrietal.(1994). The pinch analysis tools identified for integration into the MINLP framework are supertargets, interval diagrams and the $y$-y*composite curves(Hallale, 1998). For problems that feature non-linear equilibrium functions, physical insights from the McCabeThiele construction are applied to size the exchangers.

\subsubsection{The Hybrid synthesis philosophy}

The philosophy behind the hybrid synthesis method is described by Figure4.1. Most MINLP-models are non-convex and their solutions are not guaranteed to be global, therefore, targets that are based on the supertargeting technique of Hallale(1998) can be used 
to measure the optimality of MENs that are based on the MINLP models. This optimality may also be achieved through the analysis of grid-diagrams to reduce the size of the MINLP models or the driving-force plots to assess the requirements for an alternative initialisation strategy. Noting that from the conceptual design to detailed design there could be additional costs that are incurred by a MENS project, the MINLP solution are accepted even if they are $10 \%$ away from the pinch-based total annual cost targets.

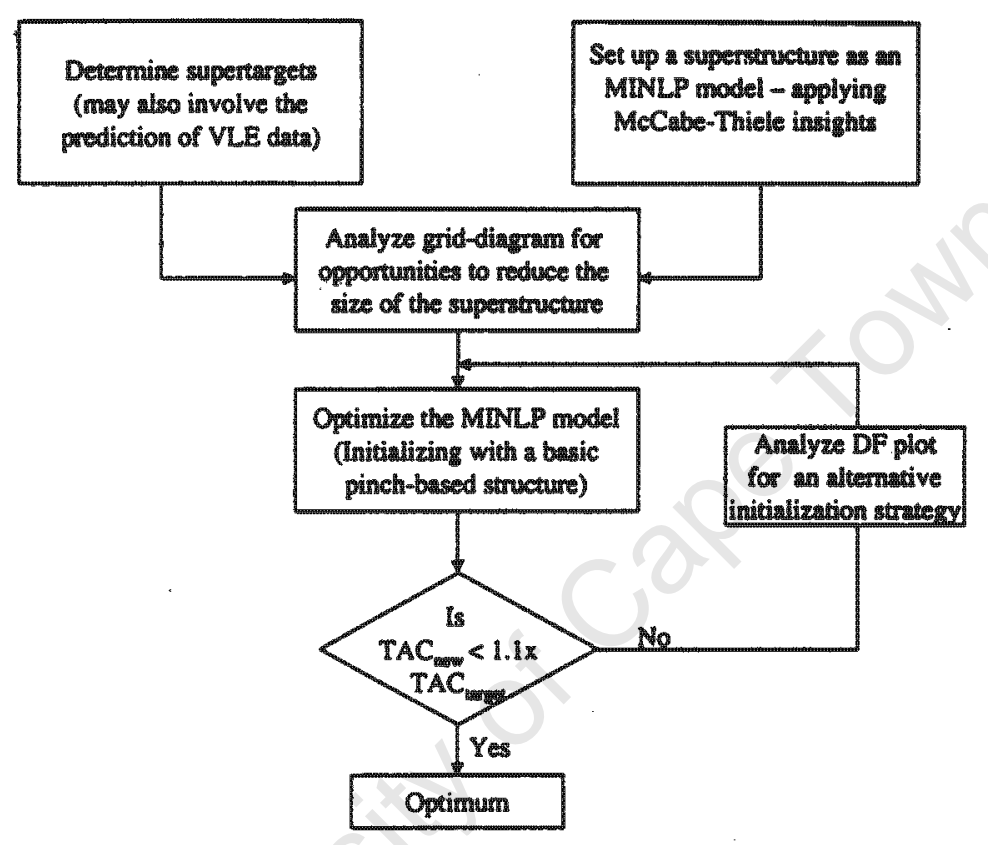

Figure 4.1: The philosophy of the hybrid synthesis method

In order to meet the design objective, the superstructure model of Papalexandrietal.(1994) is called upon and the advanced cost models of Hallale (1998) are incorporated into the model. The pinch solutions of Hallale are used as initial solutions to the MINLP solver and the targets are used to determine the need for further propagation of the MINLP solution towards the targets. This further propagation, implied by the loop in the hybrid philosophy in Figure4.1, does not represent a sequential design approach but an iterative approach whereby the designer's judgement and decisions are implemented between iterations. The allowance for the iterative method is based on the premise that completely automated design procedures could be impractical to implement or difficult to obtain in finite time. On the other hand, the many alternatives that have been exposed from the previous chapter require the use of the optimisation tool. 


\subsubsection{Problem statement}

Given are:

- A set of rich process streams with each stream $R_{i}$ having a flowrate $G_{i}$, an initial composition $y_{i}^{s}$ of the component that must be removed and its outlet composition $y_{i}^{t}$ or an upper bound $y_{i}^{U}$ for the outlet composition.

- A set of lean streams with each stream $S_{j}$ having an availability constraint that $L_{j} \leq L_{j}^{c}$, a cost $c_{j}$ associated with its consumption, an initial composition $x_{j}^{k}$ and an upper bound to the outlet composition $x_{j}^{U}$. The outlet composition is allowed to be exceeded within the network with stream mixing ensuring that the streams' final composition is within $x_{j}^{U}$. If corrosion and other safety aspects specify that everywhere within the flowsheet the upper bound should hold, then the exchanger outlet compositions should be bound as well. In addition to environmental, economic and safety constraints the outlet composition may be also be imposed by operability considerations.

- An equilibrium relation governing the distribution of a transferable component between the $i^{\text {th }}$ rich stream and the $j^{\text {th }}$ lean stream and which is independent of the presence of other soluble components in the rich stream. The effect of other species on the transfer of a key component will not be the focus of this thesis but it can be anticipated that real industrial problem will require a consideration for the incompatibility of components during the development of both the equilibrium relation and the process flowsheet.

- A set of counter-current type mass exchange technologies for each pair of rich and lean streams. Let $M P_{i j s}$ denote the set for matches that would employ packed columns and $M T_{i j s}$ for those that will employ stages vessels. Note that there is no knowledge about which pair will end up in the final network thus these sets merely classify the type of exchangers that are available.

- Also given in the problem, is an economic target $T A C^{T}$ obtained from pinch analysis as the allocated budget for the expected network.

The objective is then to synthesise a cost optimum mass exchange network, in terms of stream matches, lean stream consumption, network topology and investment cost that can 
satisfy the specifications for the lean and rich streams. The optimum network should also be one that is within $10 \%$ of the economic target.

\subsubsection{Assumptions}

The following simplifying assumptions will be used in the proposed hybrid synthesis procedure. As noted by El-Halwagi and Manousiouthakis(1990a), these assumptions can be relaxed at the expense of increased computational effort.

1. The mass flowrate of each stream remains almost unchanged as it passes through the network. This assumption holds well when there are small variations in the composition or there is counter-diffusion of the transfered component from the rich to the lean stream. Inert flowrates and mass ratios instead of mass fractions can be used in the model should the constant flowrate assumption not hold anymore.

2. All the required separation duties are based on the exchange of a single key component. This is not really an assumption but a limit on the type of problems that are considered in this thesis. There is no reason why the hybrid tool cannot be used to handle multiple components.

3. The network operates isothermally and under constant pressure. This assumption ensures that a uniform equilibrium function is used for a stream throughout the network. Later in this thesis, this assumption is relaxed when a retrofit problem involving exchangers at different pressure and temperature condition is solved.

\subsection{The Hybrid Model}

The focus of this section is on the formulation of the hybrid model that corresponds to the problem statement and the hybrid synthesis philosophy. As noted before, the formulation is similar to that of Papalexandrietal.(1994) presented in Section2.3.4. To avoid repetition, only the aspects that differ between the hybrid model and that presented in the literature review are addressed. 


\subsubsection{The size of the subnetworks}

The number of subnetworks determine the number of times that each rich stream is allowed to match with a particular lean stream. Multiple mass exchange can enhance the economics of a network while a large number of matches between streams will increase the computational effort required to solve the MENS problem. In the model, the $y-y^{*}$ interval or grid diagram is used to determine the maximum number of subnetworks according to

$$
s_{\max }=n_{\text {ist }}
$$

The $y-y^{*}$ interval diagram allows for streams to match for the number of times that there are intervals through which the lean and rich stream simultaneously exist. Thus the actual number of subnetworks will correspond to the maximum number of matches of the same stream-pair. Selecting an arbitrary small value may cut the optimal solution. MINLP problems are NP-hard problems (Floudas, 1995) and the use of too large a number of subnetworks will increase the number of binary variable combinations to be considered for the final solution and make it difficult to even find the solution in finite time. A problem is NP-hard if solving it in polynomial time would make it possible to solve all problems in class NP (Nondeterministic Polynomial time) in polynomial time.

\subsubsection{Defining stream bypass}

The definition of a stream bypass includes the stream connection between adjacent exchangers. An assumption is not made in the problem definition regarding the recycle and re-use of streams in the network. It is however, important to understand the effect of each of these phenomena (recycle or reuse) in the context of MENS in general and how it is defined in the superstructure. The variables describing the recycle or reuse of streams are $g b\left(i, j, j^{\prime}, s, s^{\prime}\right)$ and $l b\left(j, i, i^{\prime}, s, s^{\prime}\right)$ respectively. Simply put, $g b\left(i, j, j^{\prime}, s, s^{\prime}\right)$ means that when considering the flow of $i$, exchanger $(i j s)$ receives as its feed, material from exchanger $\left(i j^{\prime} s^{\prime}\right)$. Similarly, $l b\left(j, i, i^{\prime}, s_{,} s^{\prime}\right)$ means when the flow of the lean stream $j$ is considered, exchanger (ijs) receives material from exchanger $\left(i j^{\prime} s^{\prime}\right)$.

When a lean stream is recycled, it carries with it a higher composition of the transfered component. A lean stream that is rich in the desired compound reduces the available driving force for the match with a rich stream. Similarly, a rich stream that is already 
purified is re-contaminated when it is returned to a richer stream and the driving force for its match with a lean stream is decreased as well.

Consider Figure4.2 wherein stream re-use is when an exchanger that does not exchange mass exists in the final flowsheet. Streams that pass through this exchanger exit it at the same compositions that they left the last exchanger. The effect of this is that if the last exchanger is called Ex-A and the new exchanger is called Ex-B, the transportation of compound $\mathrm{p}$ from Ex-A to Ex-B is considered a transfer to an alternative exchanger even though Ex-B does not have a physical meaning. This form of re-use is problematic when stream recycle is strictly forbidden. Ex-B can intercept the outlet stream of Ex-A and thus allow for the outlet of Ex-B to in fact return to Ex-A causing an intercepted self-recycle. The intercepted self-recycles and their intermediate exchangers must be eliminated as well since these do not have any physical meaning.

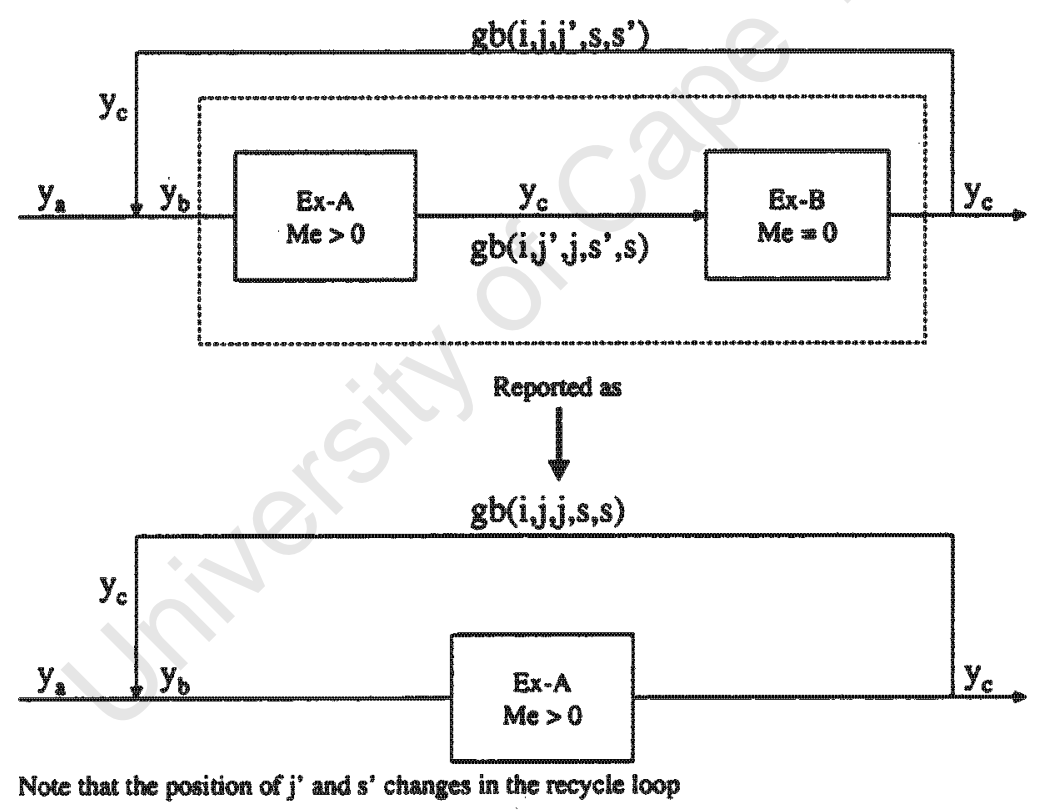

Figure 4.2: Definition of stream-bypass and stream-recycle in the MINLP model

The superstructure excludes an overall bypass where a stream goes through a network without passing through any mass exchanger. The implication of excluding an overall bypass is that the inlet composition to the technology that will accept the terminal lean stream will be lower than the target composition $x_{j}^{\ell}$ from the MEN. Overall bypass is important when the flowrate in the hybrid model is fixed at a particular value $L_{j}^{*}$. When 
not all of $L_{j}^{*}$ is required then there is an opportunity for the unused portion to bypass the network.

\subsubsection{Feasibility constraints}

The feasibility constraints in AppendixC, EquationsC.0.13andC.0.14 are treated as optimisable functions and they are allowed to take on any values greater or equal to zero according to Equations4.1 and 4.2.

$$
\begin{aligned}
& y_{i j s}^{I}-m_{j} x_{i j s}^{O}-b_{i j}=Y r I_{i j s} \\
& y_{i j s}^{O}-m_{j} x_{i j s}^{I}-b_{i j}=Y r O_{i j s}
\end{aligned}
$$

The variables $Y r I_{i j s}$ and $Y r O_{i j s}$ represent the driving force at the rich and lean end of an exchanger respectively. In the pinch notation these variables are equivalent to $\Delta y_{i j s}$ or $m_{j} \varepsilon$.

The use of a small composition difference in a match is penalised in the objective function by an increase in the capital cost whilst the use of a larger composition difference increases the external MSA requirements. Clearly there is an optimal composition difference for each match. This is a feature that has not been tested in the MINLP work of Papalexandri et al., (1994) where the minimum composition difference is in fact an optimised single $\varepsilon_{i j s}$ or $\Delta y_{i j s}$. Where this composition difference was allowed to be specific to a stream, Hallale(1998) showed that its activeness in the final network (of an example solved that was previously solved by Papalexandri et al,(1994)) led to an increased consumption of MSA.

Methods proposed in pinch analysis to deal with match-based composition differences assume that the driving force is controlled by one stream type (i.eeither the rich or lean stream). In the MINLP framework, $\varepsilon_{i j s}$ or $\Delta y_{i j s}$ is controlled by contributions from both the rich and lean streams. Within the MINLP framework, the two problems types: streamdependent $\varepsilon_{i j s}$ or $\Delta y_{i j s}$ and stream-independent $\varepsilon_{i j s}$ or $\Delta y_{i j s}$ are in fact the same. 


\subsubsection{Sizing models}

The advanced sizing models based on exchanger mass and volume (Hallale, 1998) will be tested in the hybrid model. These sizing models are summarised in AppendixB and the developments of their details are in Hallale (1998).

With regards to the Kremser equation, Equation2.10 is used instead of the highly nonlinear form of Equation2.9. It is clear from Equation2.10 why Papalexandri et al. (1994) used a positive value of $\varepsilon_{i j}$. It ensured that the logarithmic term was determinable. The use of a fixed $\varepsilon$ requires that the optimisation solution be analysed for exchangers that could be trapped at this lower bound. The bounds should then be lowered until they are not active for the exchangers that end up in the final design. This approach of reducing $\varepsilon$ is different to the method of fixing $\varepsilon$ to a lower bound as used in the Papalexandri model. The reasons for using $\varepsilon$ are, however, the same-i.eto ensure that the solution search is not hindered.

The binary approximation, quadratic constraint or $N_{s t}$-summation operators can be used for the selection of the appropriate form of the Kremser equation.

\subsubsection{Minimising non-linearities}

Fractions, logarithmic and exponential relations are expressions whose non-linearity should be minimised by the use of common denominator multipliers, slack, transpose and linearisation operators to reduce the computational effort. Common denominator multipliers allow quotients to be formulated as bi-linear terms according to Equations4.3and4.4.

$$
\begin{aligned}
y & =\frac{A}{B} \\
B y & =A
\end{aligned}
$$

Transpose and linearisation operators allow for complex expressions occurring in quotients or logarithmic relations to be expressed as intermediate variables. These variables have clearly identifiable ranges or bounds. Where the range of the intermediate variables is not known, slack operators are called upon to reduce or prevent potential infeasible solutions during the optimisation process. An example of a complex expression is the Kremser equation where the logarithm in the numerator of Equation2.9 contains the optimisable 
driving force expression. To prevent the illegal operation of determining the logarithm of a zero driving force, the slack operator is added to the driving force ratio. An example of a compact form of Equation2.10 is given by Equation4.5 where the contents of the logarithm in the numerator are lumped into the positive intermediate variable $Y r_{i j s}$

$$
N_{s t, i j s} \ln A_{i j}=\ln \left(Y r_{i j s}+10^{-6}\right)
$$

\subsubsection{Logical constraints}

The logical constraints ensure that only matches that exchange mass are reported in the final network. They are formulated according to the big- $M$ method described by Equations2.18and2.19. Thus

$$
\begin{aligned}
M_{e, i j s}-Y_{i j s} U^{M e} & \leq 0 \\
\text { where } \quad U_{i j s}^{M e} & =\min \left\{M e_{j}^{u p}, \quad M e_{i}^{u p}\right\}
\end{aligned}
$$

The value of the big-M parameter (in this case $U_{i j s}^{M e}$ ) is the minimum of the maximum amount of mass that a rich or a lean stream can exchange. In most MENS problems, this minimum is determined by the rich stream since an infinite amount of MSA is usually assumed to be available. As for the other connectivity constraints described by EquationsC.0.16toC.0.23 the parameter $U$ must be made big enough to get a feasible solution for the NLP solver.

\subsubsection{Initialisation strategy}

An addition to the MINLP model is an initialisation strategy - a pinch solution is used to initialise the variables in the hybrid model. It is well known that in order to achieve near optimal non-linear solution, a good starting point is required by the solver. It is not clear from the Papalexandri model how different initial solutions affect the final flowsheet. It could be argued that pinch-based solutions are optimal already and thus the use of computational intensive MINLP methods is not justifiable. MINLP models provide the designer with a total problem assessment tool to investigate how far various considerations 
in problem specifications drift the objective function away from or close to set targets. This simple model expansion capability is one of the prime advantages of using MINLP tools to handle problems with complex multi-variable trade-offs.

In the absence of pinch solutions various other initialisation strategies need to be explored in order to provide a feasible initial solution to the NLP solver. Note that the CONOPT algorithm can find subsequent NLP solutions fast and thus the modeller needs to provide the initial solution only at the start of the solution search. Examples of these initialisation strategies are:

- A flowsheet with all the mass transfer requirements met by an external MSA. Stream splits can be considered to account for initial flowsheets with series or parallel connections;

Drawing from experiences in HEN, cold and hot utilities alone can complete the heat exchange duty of a HEN and the network can be considered feasible. Similarly, an external MSA may be employed to complete the mass exchange duty and the resulting solution be deemed feasible.

- A flowsheet with each of the process MSAs exchanging maximum mass with a rich stream and the rest of the transfer completed by the external MSA. The cheapest MSAs should be used first in case there is more than one external MSA;

- Initialisation based on block decomposition (Zhu, 1995); and

- Initialisation based on pinch-less grid diagram (Comeaux, 2000).

\subsubsection{Model solver}

The MINLP models in this thesis are solved via the DICOPT solver which iterates between the OuterApproximation/ExtendedRelaxation/AugmentedPenalty-based CPLEX algorithm to determine the solution of the MILP sub-problem and the GeneralisedReducedGradient(GRG)based CONOPT2 algorithm for the solution of the NLP sub-problem. Once again, the choice of the OA/ER/AP-based algorithm makes no assumption of the superiority of DICOPT over the Generalised Benders Decomposition algorithm used in Papalexandrietal.(1994). The hybrid model is implemented in GAMS on a PIl Celeron $533 \mathrm{MHz}$ PC. 


\subsection{Case Studies}

The selected cases discussed in this section have all been used in the work of Hallale (1998). They were chosen because: they have complete sets of data that make economic optimisation possible and most of the cases have already been solved via other synthesis methods. So these results can be re-used in testing the hybrid model. The case studies referred to are:

- Capital cost optimisation for the removal of $\mathrm{SO}_{2}$ from four gaseous process streams;

- Total cost optimisation for a dephenolisation process wherein the size of exchangers is based on volume;

- Consideration of regeneration networks; and

- Consideration of reactive mass exchange networks to illustrate the use of the McCabeThiele approximation.

Data for the streams and for costing purposes is given in AppendixA.

The other case studies from Hallale (1998) are considered in the PhD work of Zsolt Szitkai at the Technical University of Budapest.

\subsection{1 $\mathrm{SO}_{2}$ removal}

The first case study involves the removal of sulphur dioxide from four gaseous process streams $\left(R_{1}, R_{2}, R_{3}\right.$ and $\left.R_{4}\right)$ using fresh water $\left(S_{1}\right)$ as the external MSA. The absorption process is to be carried out in carbon steel sieve trays. The objective is to design a mass exchange network that will deliver the separation of $\mathrm{SO}_{2}$ from the gaseous streams with the minimum flow of water and with a minimum capital cost. This dual objective approach is characteristic of the capital cost targeting approach of Hallale(1998) in that the limiting amount of $S_{1}$ is determined first before the capital cost targets can be set. The current amount available for this project (determined from pinch capital cost targets) is $\$ 860000$. An initial pinch design is shown in Figure4.3.

The first task in the hybrid model is to convert the pinch solution into a form that is compatible with the superstructure variables. 


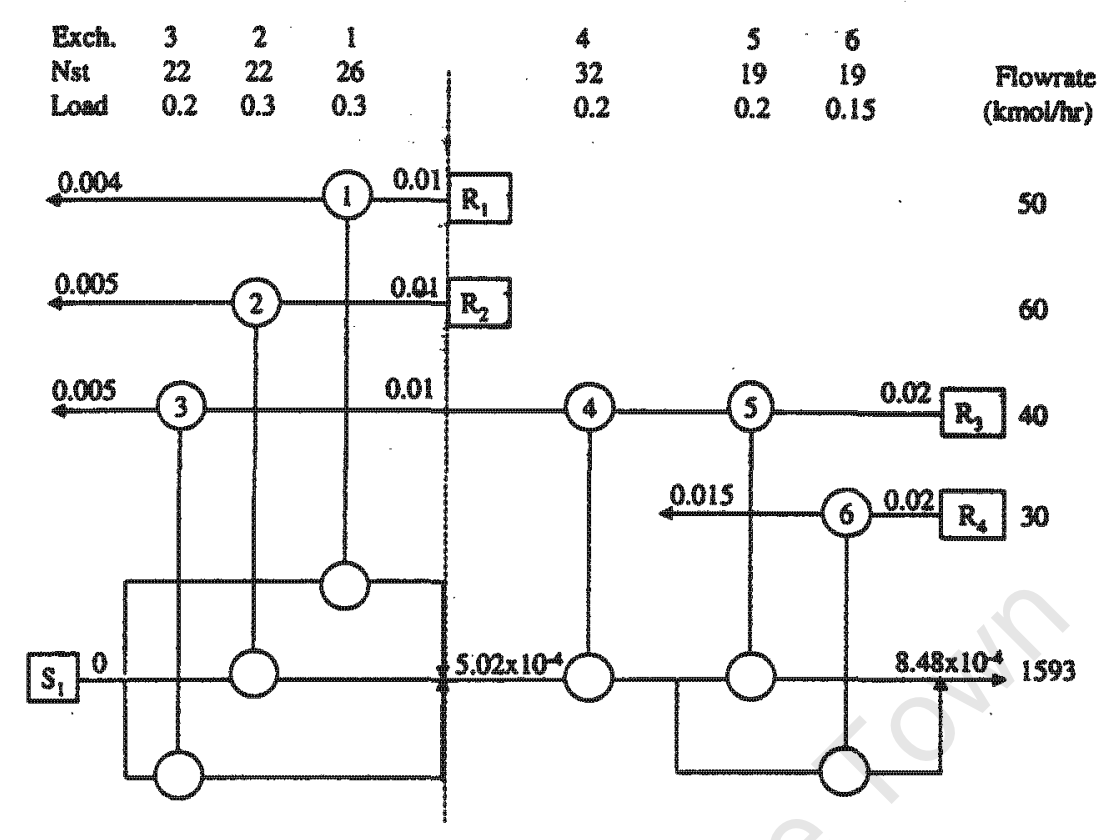

Figure 4.3: Pinch solution for the $\mathrm{SO}_{2}$ removal problem. Capital cost $=\$ 860000$

Next is the determination of the maximum mass loads that each stream possesses to use in the big-M formulation. The lean stream is available in large amounts thus the rich streams are used to get the following mass loads for the rich streams: $R_{1} 30, R_{2} 30, R_{3} 60$ and $R_{4}$ $15 \mathrm{kmol} / \mathrm{hr}$. The diameter and tray spacings used in these problems have been assumed to be similar to those used in Hallale (1998).

The final flowsheet, Figure4.4 from solving the hybrid model has a different structure when compared to the flowsheets previously reported by Hallale and Fraser (1998) for the same problem. The capital cost of the new flowsheet is $\$ 321000$, which is better than the capital cost target by $62 \%$. This reduction in capital cost is brought about by the increased consumption of fresh water in the hybrid solution. Although the objective in this problem is to minimise capital cost, during the targeting process, the limiting composite curve is used to also determine the minimum amount of water that is required to remove $\mathrm{SO}_{2}$. In the hybrid model there is no penalty incurred for using extra water thus the freshwater consumption is increased so that the capital cost targets can be met or even beaten. The use of mass or volume-based sizing models, more especially in the hybrid model should influence the amount by which an MSA may increase in order to minimise the capital cost. This influence is based on the fact that, unlike the number of stages (determinable 
by using compositions only), mass or volume-based models depend on the flowrate of the exchanging streams as well.

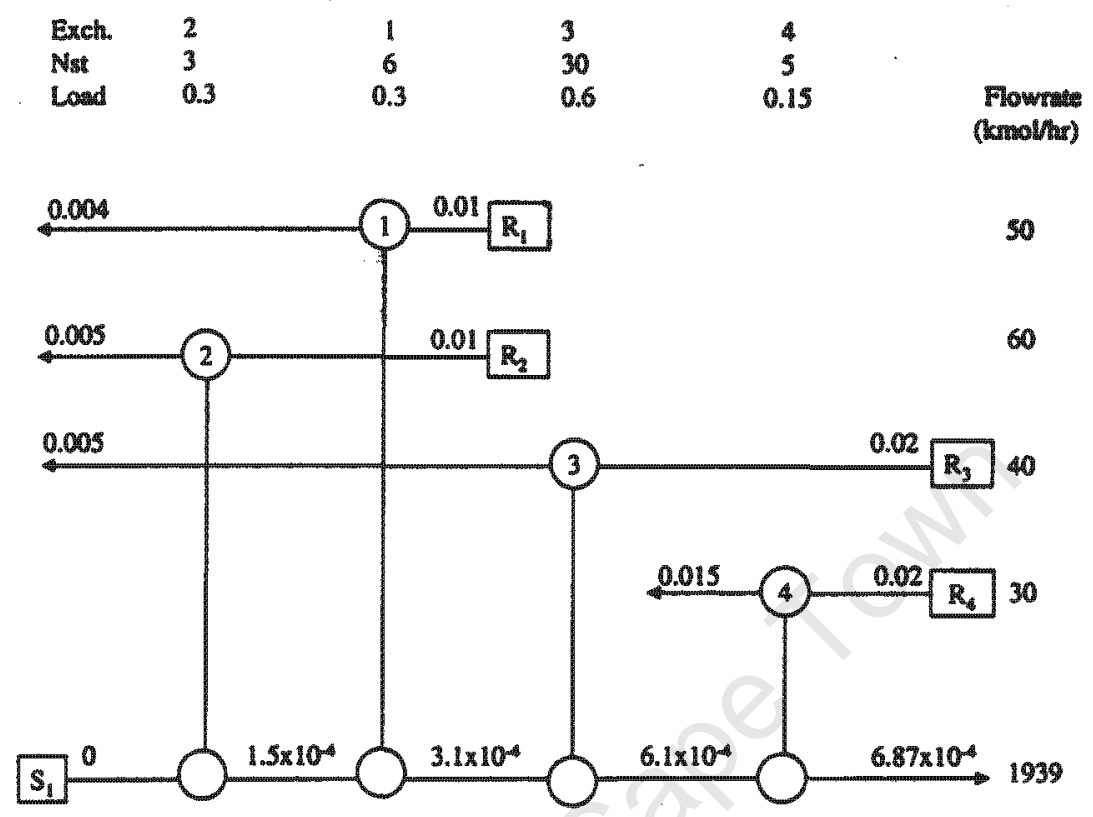

Figure 4.4: Hybrid solution for the $\mathrm{SO}_{2}$ removal problem. Capital cost $=\$ 321000$

Supposing that because of the presence of foaming components, the use of tray columns is no longer appropriate and packed columns have to be used for the separation. The foaming components are present in the MSA which which now also contains some $\mathrm{SO}_{2}$. To implement this change, the hybrid model simply requires the modification of the inlet composition of the lean stream and the use of an appropriate sizing-expression in the objective function. In pinch analysis the $\mathrm{SO}_{2}$ content, $x^{s}$, in the MSA entering the network would mean re-working the targets, constructing the interval diagram, generating the initial flowsheet and evolving it to an optimal solution.

In order to retain use of the targets to evaluate the hybrid models, it is nevertheless, still necessary in this example that the capital cost targets be determined for the packed column exchanger as well. The capital cost target adopted for evaluation of the hybrid solution is $\$ 448000$ and the hybrid solution must approach it to within $10 \%$. For comparison with the MINLP solution, a pinch design that was generated by Hallale(1998) to meet the $\$ 448000$ capital cost target is given by Figure4.5(a)

Figure4.5(b) shows a hybrid network that costs $\$ 488000$ which is within $9 \%$ of the target. 
A closer look at the basis for the poor hybrid solution shows that there is a large increase in the MSA flowrate in the MINLP solution compared to the pinch solution.

\section{Remarks}

The $\mathrm{SO}_{2}$ removal problem has confirmed the limitations of using only one type of cost in the objective function. The capital cost is minimised at the expense of the consumption of MSAs. Vice versa the flowrate of MSAs is minimised at the expense of large mass exchange units. The reduction in capital investment subject to increased MSA flowrates cannot be justified if the capital cost model does not take into account the requirements for equipment and facilities that need to be made available to handle the additional MSA.

It is noted that in the pinch approach, a capital cost is minimised subject to the network meeting a minimum consumption of MSA (set by the water pinch or minimum composition difference). This approach is not different to the problem of minimising total cost at a. fixed minimum approach composition except that the capital cost targets are known before design. The MINLP approach on the other hand allows the MSA consumption to be traded-off with capital cost according to the weighting that is placed on the operating and capital cost in the objective function. Comeaux (2000) compares these un-optimised pinch solutions to an NLP approach thus using an unfair basis to compare the synthesis techniques. The supertargeting solutions should be the ones that are used to compare the performance of any alternative synthesis technique.

\subsubsection{Dephenolisation}

In the dephenolisation of two aqueous streams, $R_{1}$ and $R_{2}$, by solvent extraction, gas oil ( $\left.S_{1}\right)$ and lube oil $\left(S_{2}\right)$ are available as process MSAs. Light oil $S_{3}$ is used as an external MSA. The total annual budget for the anticipated flowsheet is $\$ 226000$ with $\$ 158400$ contributing to the annual operating cost and $\$ 338000$ (annualised by multiplying by $20 \%$ ) accounting for the capital available for the implementation of the project. The hybrid flowsheet must therefore produce a network that will be within $10 \%$ of this budget. The flowsheet is to employ staged vessels for the separation process. The objective function for this problem is based on the exchanger volume given by Equation4.6. 


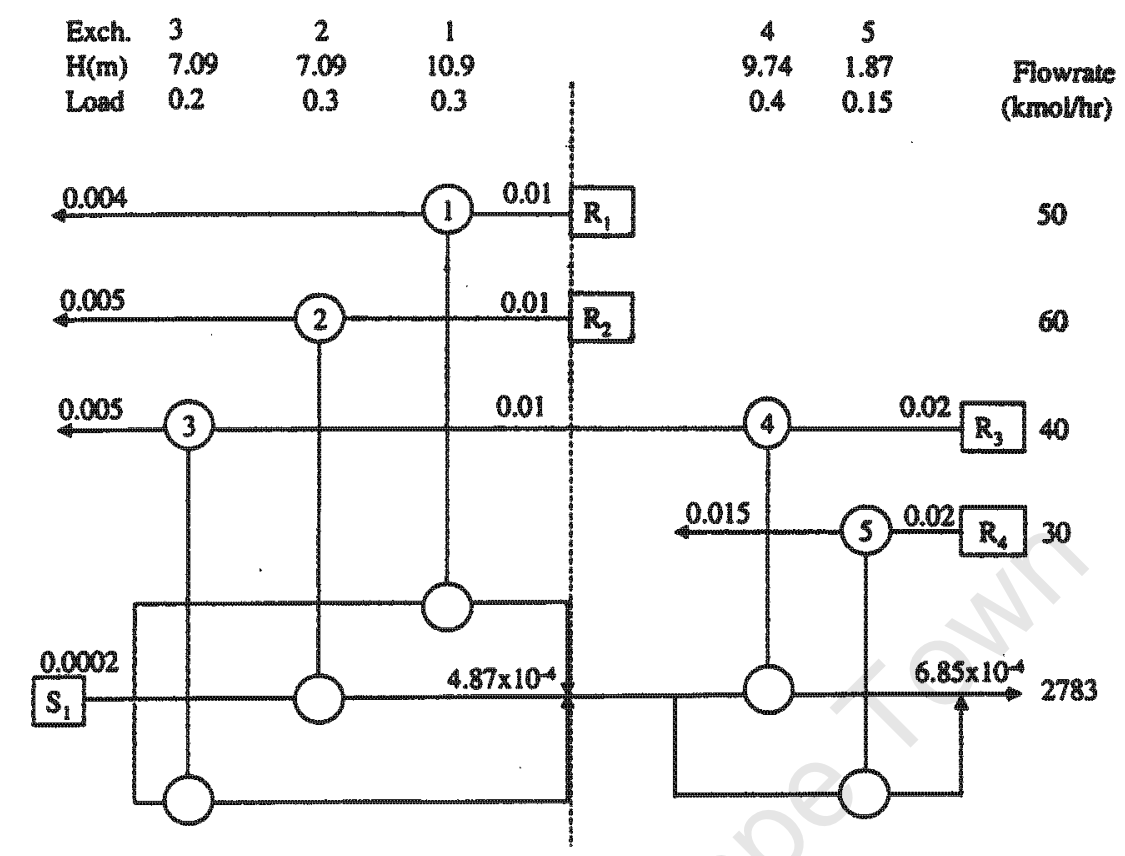

(a)

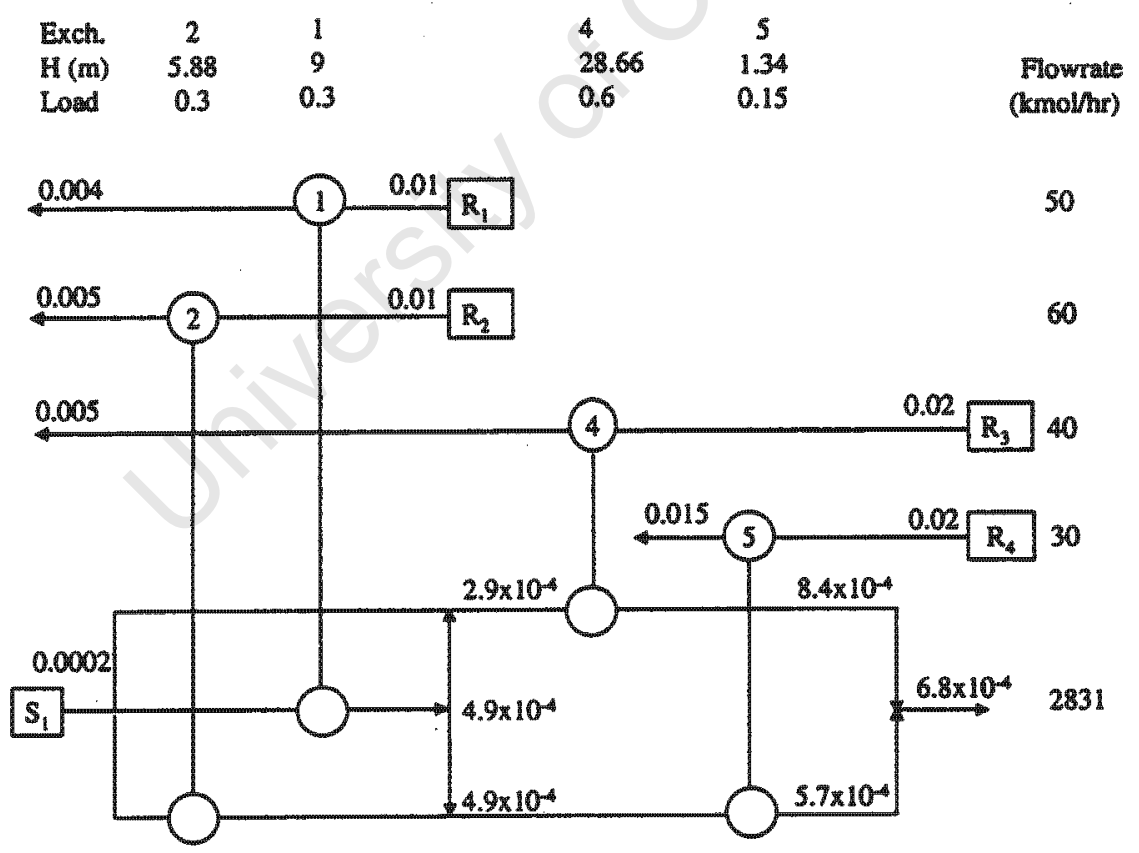

(b)

Figure 4.5: A pinch (Capital cost $=\$ 448000$ ) (a) and a hybrid-based (Capital cost $=$ $\$ 488000$ ) (b) solution for $\mathrm{SO}_{2}$ removal problem when considering packed columns 


$$
V_{i j s}=\frac{\tau N_{s t, i j s}}{E_{o}}\left(\frac{g_{j j s}^{E}}{\rho_{i}}+\frac{l_{i j s}^{E}}{\rho_{j}}\right)
$$

$\tau$ is the residence time in seconds per stage

$E_{0}$ is the separation efficiency. It has been assumed to be one.

First, the size of the model is considered in terms of the number of subnetworks. According to Figure4.6 the number of subnetworks should be 4 due to the potential of multiplematches between $R_{1}$ and $S_{2}$. The maximum number of potential matches in the superstructure at the beginning is 42 according to Equation 4.7 which multiplies the number of streams with the number of intervals. The equality will be active if all the streams exist in all the intervals in Figure4.6. When the number of subnetworks is used instead of the number of intervals, the number of potential matches reduces to 24 .

$$
n_{\max } \leq 2 \times 3 \times 7
$$

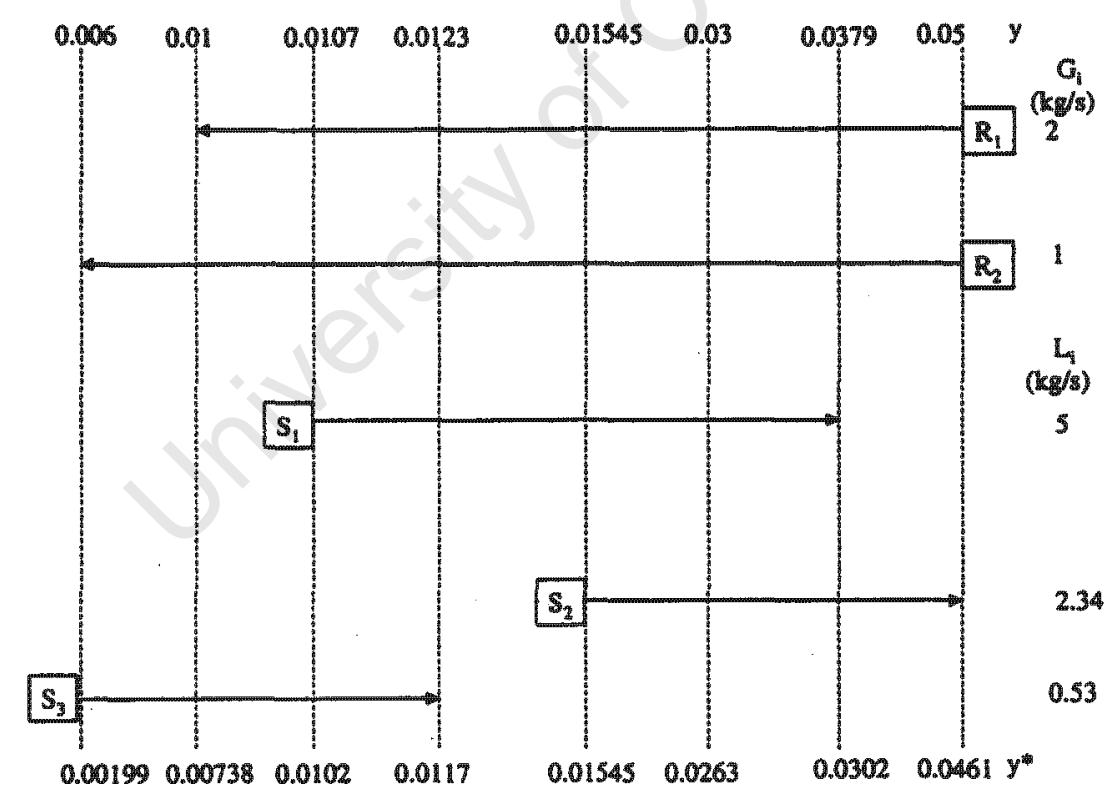

Figure 4.6: Grid diagram for dephenolisation example

The hybrid design is highly sensitive to the starting network, so a pinch solution (Figure4.7) is used to initialise the NLP solver. While it is well known that pinch-based solutions are near optimal and one might assume that MINLP solvers should find it easy to reproduce 
or improve the pinch solution if it is used as an initial solution, this is found to be not the case. The MILP makes linear cuts on the nonlinear space obtained from the solution of the non-linear (primary) subproblem. These integer cuts change the path or direction of the solution search and thus an alternative solution is reported. The final solutions are however structurally similar no matter what little modification is made to the cost of the initial solution. This makes the requirement for MINLP solutions to be within $10 \%$ of the targets a reasonable constraint and attempting to make the MINLP solution to be exactly on target may be an objective that cannot be met in finite time.

The hybrid solution for the dephenolisation problem is given in Figure4.8 and has a total annualised cost of $\$ 235000 / y r$ which is within $5 \%$ of the target.

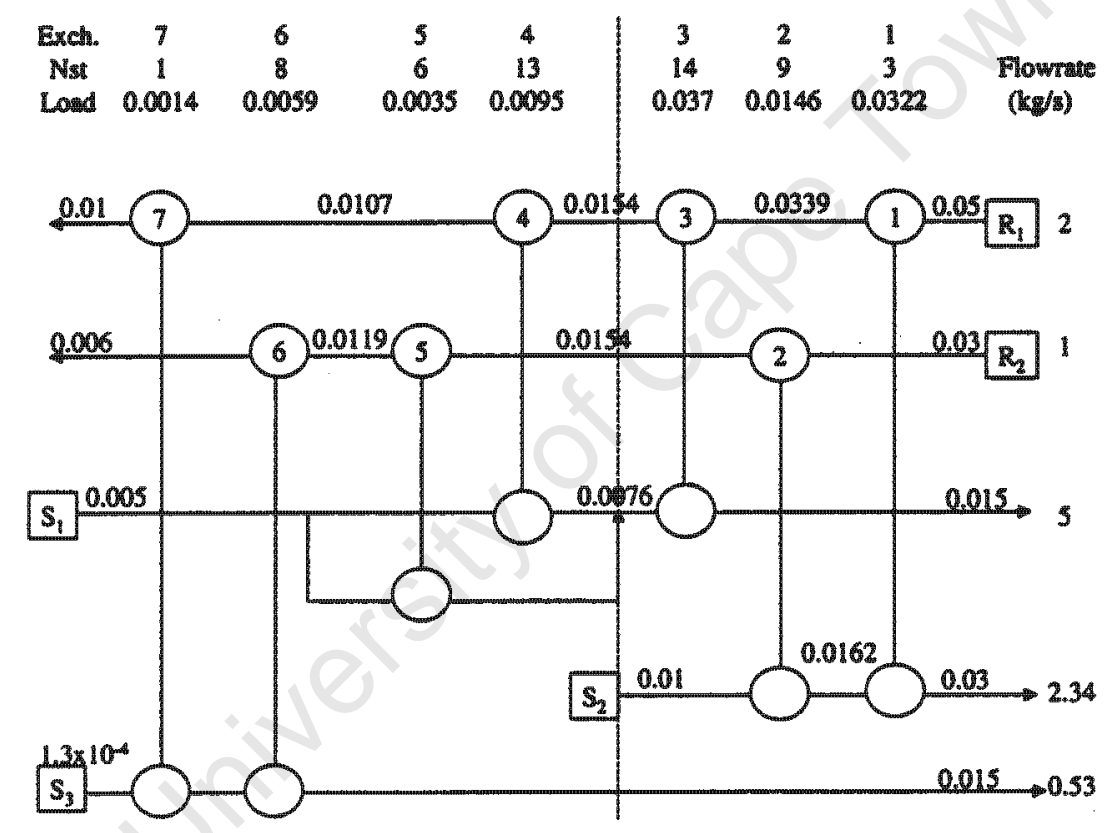

Figure 4.7: Optimum pinch solution for the dephenolisation example. Capital cost $=\$ 158400 \mathrm{k}$, Operating cost $=\$ 67000$

The network structure is similar to the pinch solution except for the reduced circulation rate through exchanger 2 in Figure4.8. The hybrid solution is achieved through the simultaneous optimisation of the capital and operating cost and the generation of a network structure.

Different stream properties, e.g corrosivity, temperature, pressure, etc, may require the use of different types of material of construction whilst purity, residence time, etc requirements may require the use of different exchanger types within the same network. In the next example, the use of a different external MSA in the dephenolisation problem requires the 


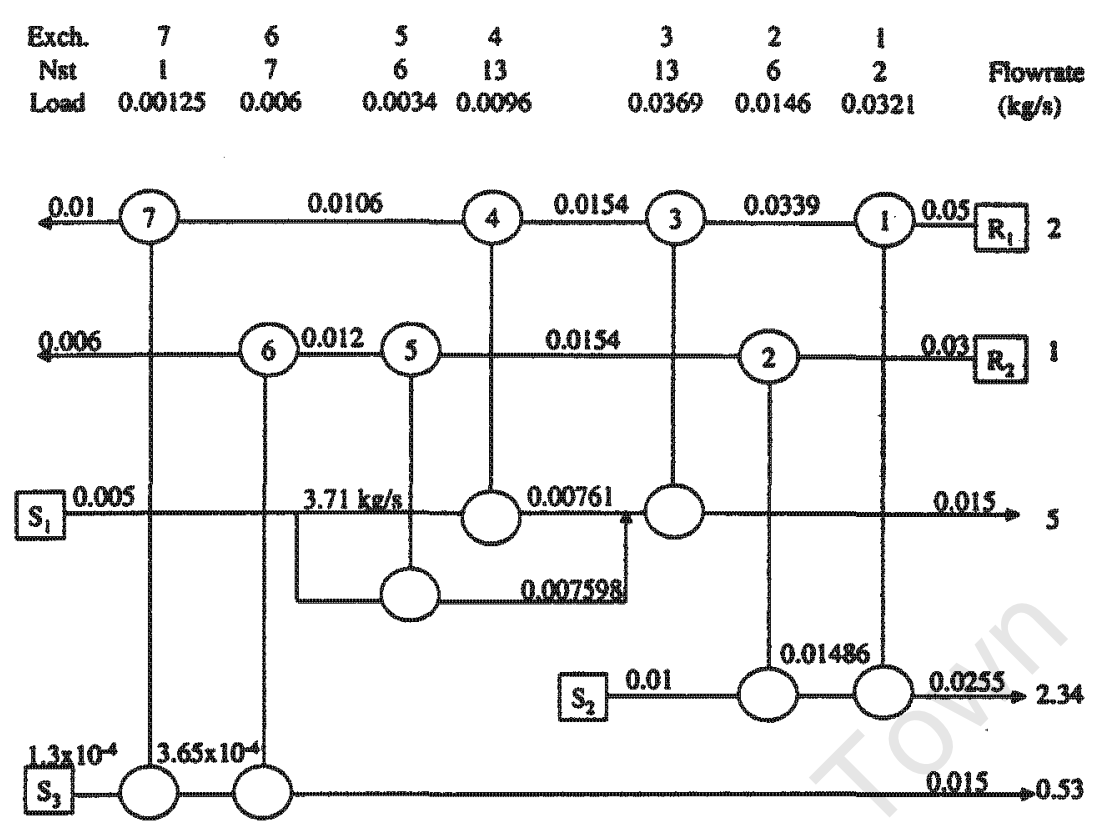

Figure 4.8: Optimum hybrid solution for the dephenolisation example. Capital cost $=$ $\$ 235000$, Operating cost $=\$ 158400$

use of trayed stripping columns for $S_{3}$ and mixed-settlers for the process MSAs $\left(S_{1}\right.$ and $\left.S_{2}\right)$. All the exchangers that involve the aqueous rich stream $R_{2}$ must be made of stainless steel due to the corrosive property of this stream. The exchanger classification for this example is given in Table4.1.

Table 4.1: Exchanger classification table for the mixed equipment-type example

\begin{tabular}{|c|c|c|}
\hline & $R_{1}$ & $R_{2}$ \\
\hline \hline$S_{1}$ & Carbon steel mixer-settlers & Stainless steel mixer-settlers \\
\hline$S_{2}$ & Carbon steel mixer-settlers & Stainless steel mixer-settlers \\
\hline$S_{3}$ & Stainless steel tray columns & Stainless steel tray columns \\
\hline
\end{tabular}

A pinch-based solution reported a network design having a TAC $\$ 526,000 / y r$ based on a supertargeted value of $\$ 524,000 / y r$. Figure4.9 illustrates the effect of mixed equipment type on the MEN cost. The network has a total annual cost of $\$ 552000$ which is within $6 \%$ of the given targets. 


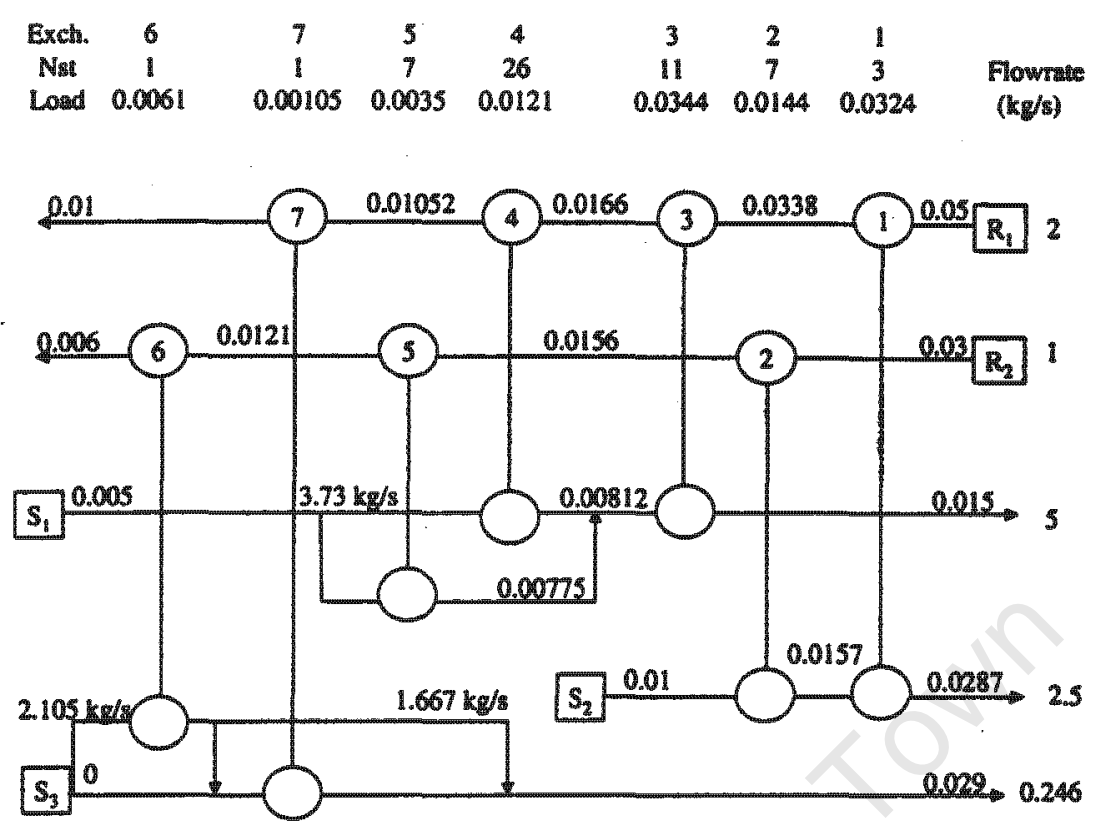

Figure 4.9: Hybrid solution for the mixed equipment-type example. Capital cost $=$ $\$ 119000$, Operating cost $=\$ 433000$

\section{Remarks}

Experimentation has shown that the proposed method for calculating the number of subnetworks and hence the binary variables is not consistent. Sometimes a smaller number of subnetworks is necessary. This number can be be determined by first solving the model with the maximum number of subnetworks according to Equation 4.7 and then observing the number of multiple matches between streams. Then the actual number of subnetworks for the final optimisation will be given by Equation4.8.

$$
n_{\text {optimum }}=1+n_{\text {from previous solution }}
$$

The hybrid model can handle problems with complex cost models. The MINLP is useful in handling equations of the form Cost $=a+b V^{c}$ where the constant $a$ is multiplied by an integer that represents the existence of a match. In the dephenolisation example, $a$ was always zero. Where $a$ was given as 1 , the reported number of binary variables were minimised and this eliminated the generation of units that did not exchange mass as it was cautioned in Section4.2.2. 
The changes to an MINLP model can be easily implemented to study the effect of other factors to the objective function. The dephenolisation example has used the same MINLP framework to model the problem of streams that are constrained to a particular type of an exchanger.

\subsubsection{Regeneration}

Regeneration is an important process in mass exchange networks. After a lean stream has been fully loaded with the transfered species, it becomes necessary to separate the transfered species from the rich MSA and re-use the MSA. This discarding process, may require the use of either another MSA or an energy utility. The choice of which regeneration technology to use becomes an additional question to consider in MENS. Accordingly, in the MINLP model, the regeneration section requires a secondary superstructure to be incorporated into the the main or primary superstructure. Additional variables and mass balance equations are used to describe the new superstructure thus leading to a larger model.

The regeneration example considered here involves the removal of phenol from four aqueous waste streams, $R_{1}$ to $R_{4}$, produced by a coal conversion plant. Phenol is a valuable chemical feed-stock and there is an economic incentive to recover it from waste streams (El-Halwagi and Manousiouthakis, 990b). Two MSAs, $S_{1}$ and $S_{2}$, are available for the phenol removal process. $S_{2}$ is an activated carbon stream and should be regenerated with caustic soda, $H_{1}$. Tray columns are to be used for $S_{1}$, while packed columns are to be used for $S_{2}$ and regeneration.

The pinch-based solution illustrated in Figure4.10 was used to initialise the hybrid model for the regeneration problem. The TAC target for this project is $\$ 692000$.

In the formulation of the MINLP model, each of the primary and the secondary superstructures have their own number of subnetworks. The number of subnetworks for the secondary superstructure is 1 since there is only one regeneration agent and a single match between $S_{2}$ and $H_{1}$ is to be considered. There is no reason why the method leading to Equation 4.8 cannot be used when the number of lean streams to be regenerated and the number of regeneration agents is more than 1.

The hybrid-based solution is illustrated by Figure4.11. The network costs $\$ 720000$ which is within $4 \%$ of the target. 


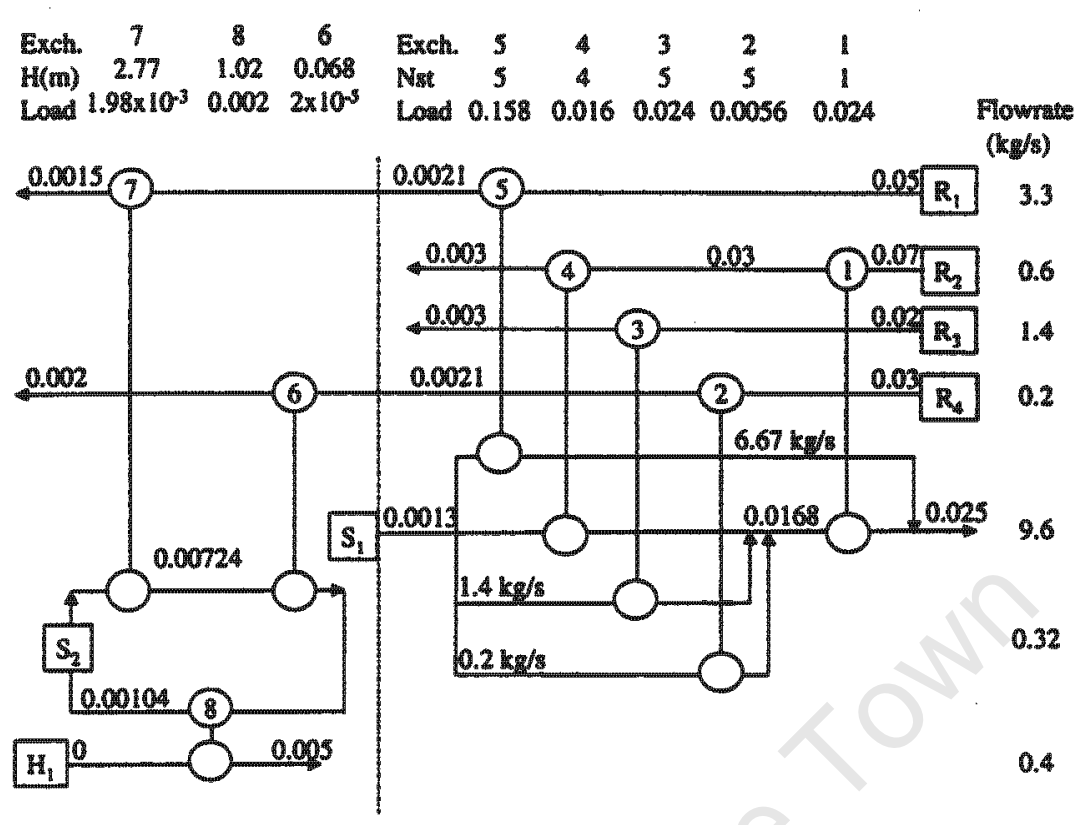

Figure 4.10: Pinch based regeneration network. Capital cost $=993500$, Operating cost $=$ $\$ 598500$

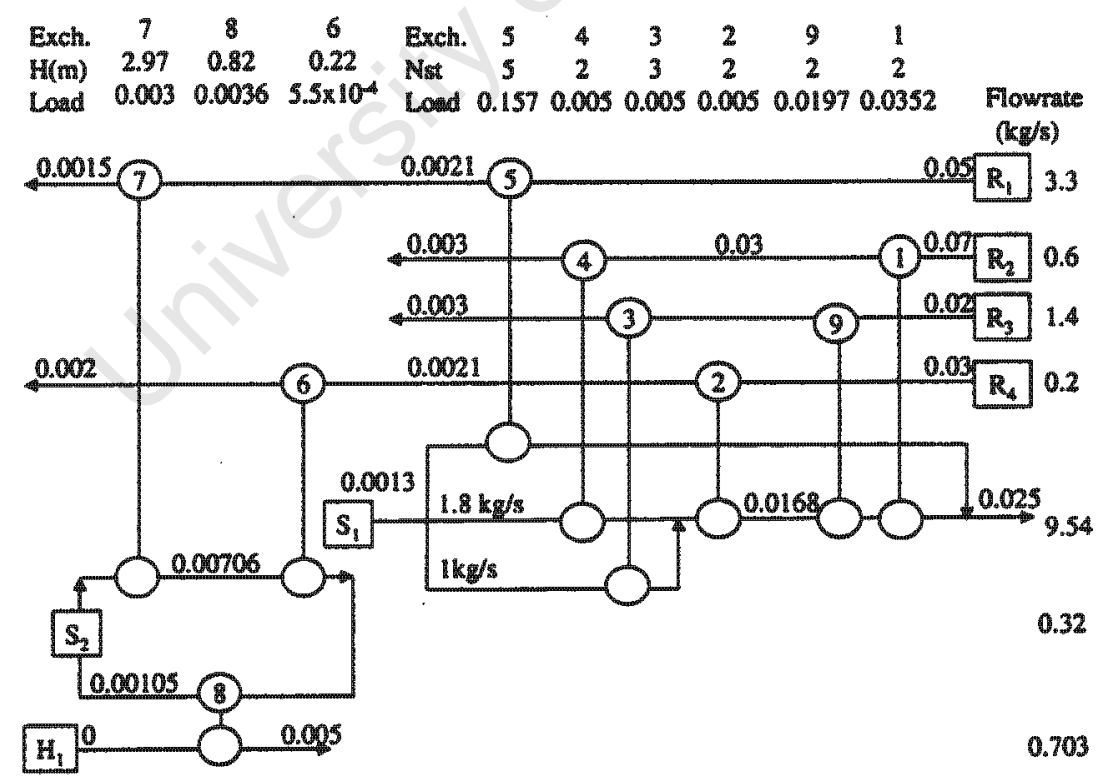

Figure 4.11: Hybrid regeneration example. Capital cost $=\$ 98300$, Operating cost $=$ $\$ 621700$ 


\section{Remarks}

Hallale (1998) relates the regeneration network to the primary network through $\varepsilon$ in that: the load of the regenerable stream $S_{2}$, at a specified composition difference is the same as the load of the regenerating agent $H_{1}$. This does not, however, mean that $\varepsilon$ should be used to determine the optimal operating conditions of the regeneration exchanger. The cost of this exchanger is still a function of the regenerant flowrate and the driving force. This driving force cannot be the same as that of the primary network. For the case where a single $\varepsilon$ is used for both networks, an exhaustive numerical search is carried out to get the optimum. This will obviously be complicated by the considerations of an additional optimisable $\varepsilon$ for the regeneration network alone. These trade-offs are well handled by the hybrid framework while the targets obtained from the pinch analysis are used to evaluate the success of the hybrid solution.

\subsubsection{Reactive mass exchange networks}

The problem of reactive mass exchange networks was previously solved for a network that featured packed columns(El-Halwagi and Srinivas, 1992). The main difference with this type of network is that the equilibrium relation between the composition of the transfered species in the lean phase and composition in the rich phase is a non-linear one. The non-linearity is brought about by the conversion of the transfered species to form other compounds using reactive MSAs. The equilibrium relation is given by Equation 4.9 where $x_{j}$ represents both the physically dissolved and chemically combined forms of the transfered species.

$$
y_{i} *=f\left(x_{j}\right)
$$

\section{Sizing packed columns}

In the next example, $H_{2} S$ is removed from two aqueous streams $R_{1}$ and $R_{2}$ which result from the production of rayon. Caustic soda $\left(S_{1}\right)$, diethanolamine $\left(S_{2}\right)$ and activated carbon $\left(S_{3}\right)$ are the available MSAs. The equilibrium relations for the MSAs is described by the following relations:

Caustic-soda $y=1.945 e-9\left(10^{0.529 x_{1}}\right)$ 
Diethanolamine $y=7.7545 e-4\left(x_{2}\right)^{2}$.

Activated-carbon $y=0.015 x_{3}$

Packed columns with a diameter of $1 m$ are to be used for the separation. The exchangers have an annualised capital cost of $\$ 9,500 / y r$ per metre of packed height.

In Hallale (1998), only the caustic soda was required in the final design. Since this MSA is free, the TAC was just a contribution from the capital cost target of $\$ 28,000$ compared to the $\$ 11273500$ reported by Papalexandri et al. (1994). The supertargeting results revealed that the constraints placed on the minimum composition difference in the MINLP framework forced the MINLP-based model to include the other two expensive MSAs in the final flowsheet. A hybrid based-network however, shows a TAC of $\$ 32000$ which is an improvement on the MINLP structure and it is also within $5 \%$ of the supertarget. The availability of TAC targets has allowed the selection of better MINLP results.

Table 4.2: Minimum allowable composition differences for the streams in the $\mathrm{H}_{2} \mathrm{~S}$ removal

\begin{tabular}{|c|c|c|c|c|c|}
\hline Stream & $\varepsilon_{1}^{S}$ & $\varepsilon_{2}^{S}$ & $\varepsilon_{3}^{S}$ & $\varepsilon_{1}^{R}$ & $\varepsilon_{2}^{R}$ \\
\hline \hline$\varepsilon\left(\mathrm{kmol} . \mathrm{m}^{-3}\right)$ & 3.5 & 0.014 & $10^{-6}$ & $10^{-7}$ & $1.5 \times 10^{-7}$ \\
\hline
\end{tabular}

If the restrictions on the minimum composition difference are kept as in Papalexandrietal.,(1994), it is possible that the hybrid solution would still produce a cheaper solution than the MINLP method because, none of the $\varepsilon$ values (seeTable4.2) reported by El-Halwagi and Srinivas(1992) and used by Papalexandrietal.,(1994) are more than $3.9 \mathrm{kmol} / \mathrm{m}^{3}$ as claimed by Hallale(1998) to be the $\varepsilon$ amount that lead to the poor MINLP solution for this example. The success of the hybrid solutions to be cheaper would be enhanced by the availability of targets which, according Hallale(1998), showed that for that $\varepsilon$ values that are less than $3.9 \mathrm{kmol} / \mathrm{m}^{3}$ only $\mathrm{S}_{1}$ (which is free) will contribute to the operating cost portion of the TAC of the network.

\section{Sizing staged vessels}

Suppose that the same example is solved this time with only monoethanolamine (MEA) used as the MSA. The desired technology now is the staged vessels which require the determination of a number of stages to size the mass transfer units. Chemical absorption is responsible for the removal of $\mathrm{H}_{2} \mathrm{~S}$ by MEA and according to Srinivas and El-Halwagi 


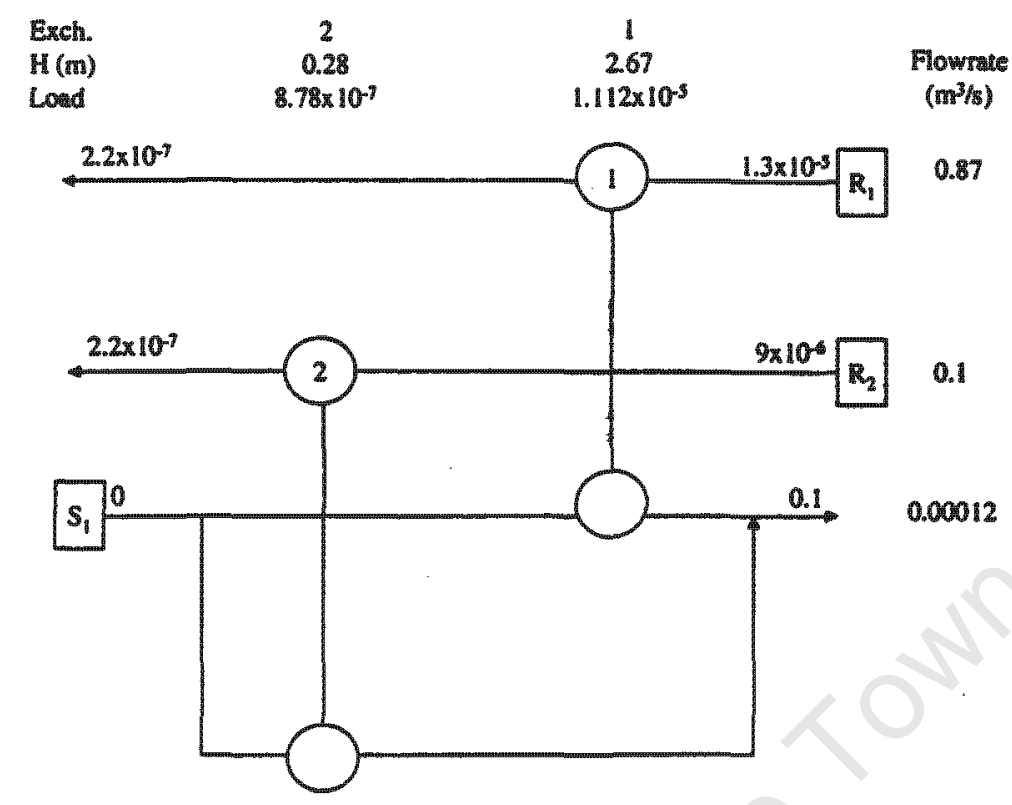

Figure 4.12: The pinch solution for the $\mathrm{H}_{2} \mathrm{~S}$ removal case. Capital cost $=\$ 28000$, No operating cost

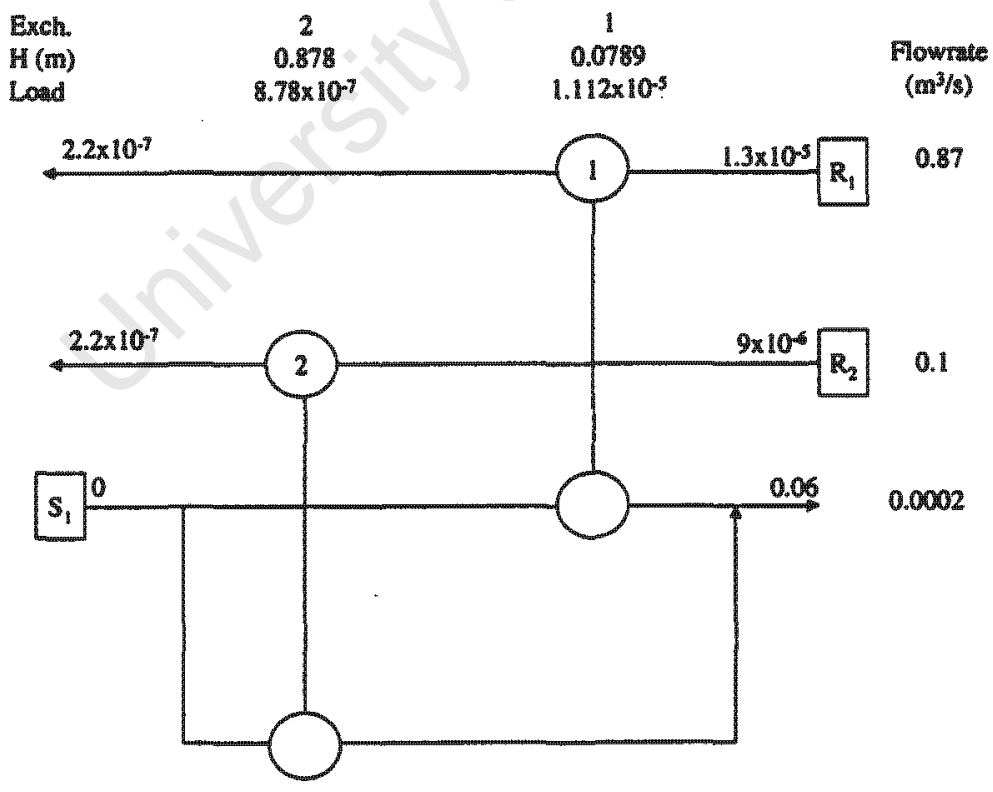

Figure 4.13: Hybrid solution for the $\mathrm{H}_{2} \mathrm{~S}$ removal problem. Capital cost $=\$ 32000$, No operating cost 
(1994) the overall equilibrium relation for this system is given by Equation4.10 and the operating line is given by Equation4.11.

Hallale (1998) solved this problem by determining the number of stages of the exchangers in the final network using the McCabe and Thiele method. The cost target for this project is $\$ 591000$. A pinch-based designed costs $\$ 535000$ which is $9.5 \%$ below the target. The low capital cost result from the fact that the cost targeting assumes equal mass distribution among the units(see Figure4.14).

$$
y(x)^{*}=-197.19 x^{3}+23.722 x^{2}-0.3248 x+0.0009928
$$

$$
y(x)=\frac{l_{i j s}^{e}}{g_{i j s}^{e}}\left(x-x_{i j s}^{I}\right)+y_{i j s}^{o}
$$

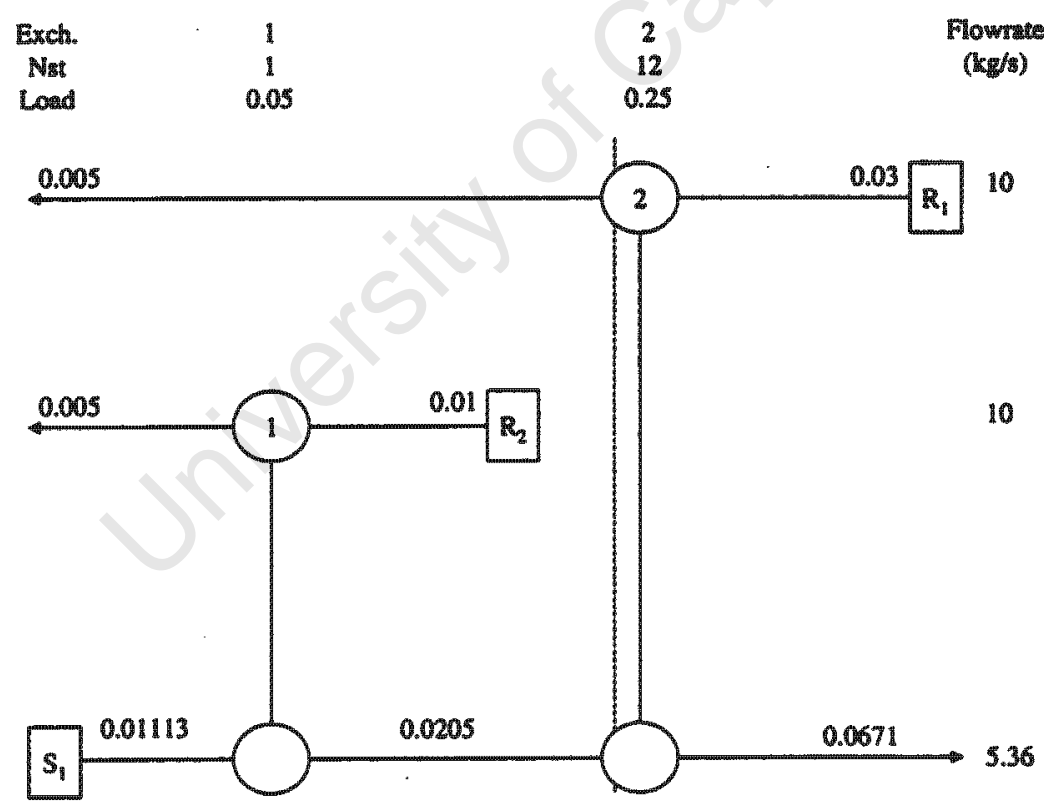

Figure 4.14: Network design based on pinch analysis. Capital cost $=\$ 535000$, No operating cost

The sizing equations in the hybrid model require the adoption of the McCabe-Thiele approach developed in Section3.2 to determine the number of stages of the exchangers. For a polynomial function, the expansion of the integral in the determination of the average 
driving force described by Equation3.7 will be given by Equation4.12:

$$
N s t_{i j s}=\frac{\left(y_{i j s}^{I}-y_{i j s}^{O}\right)}{\frac{1}{2} o m_{i j s}\left(x_{i j s}^{O}+x_{i j s}^{I}\right)+o b_{i j s}}-\frac{\left(y_{i j s}^{I}-y_{i j s}^{O}\right)\left(x_{i j s}^{O}-x_{i j s}^{I}\right)}{\frac{a_{1}}{b_{1}+1}\left[\left(x_{i j s}^{O}\right)^{b_{1}+1}-\left(x_{i j s}^{I}\right)^{b_{1}+1}\right]}-\ldots
$$

where $o m_{i j s}$ and $o b_{i j s}$ are the slope and the intercept respectively of the operating line $y(x)$ described by Equation4.11. The constants $b_{1}, b_{2}, \ldots, b_{n}$ and $a_{1}, a_{2}, \ldots, a_{n}$ represent respectively the power and coefficients of the polynomial describing the equilibrium relation of the reacting species.

Figure4.15 illustrates the solution of this particular case.

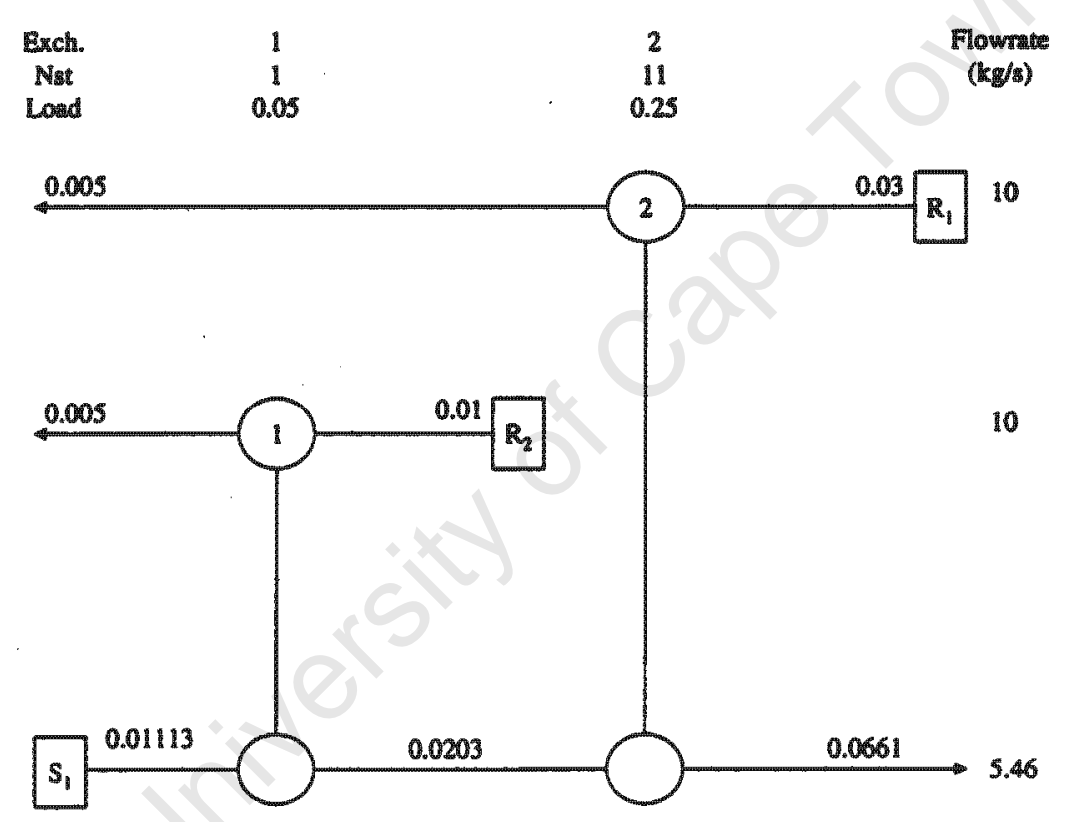

Figure 4.15: Proposed reactive mass exchange network with the sizing based on the developed approximation method. Capital cost $=\$ 512000$, No operating cost

The proposed solution features a similar network to that obtained by pinch methods (Hallale, 1998). This illustrates the usefulness of the McCabe-Thiele approximation to size staged vessels when the Kremser equation cannot be used.

Table4.3 compares the results of the MTA method with the McCabe-Thiele construction results.

The approximation is consistent and gives results that are close to those obtained from the McCabe-Thiele construction. The difference in fractions is brought about by not 
Table 4.3: Number of equilibrium stages obtained for the reactive mass exchange network.

\begin{tabular}{|c|c|c|}
\hline Calculation method & Exchanger 1 & Exchanger 2 \\
\hline \hline McCabe-Thiele & 0.62 & 10.33 \\
\hline MTA & 0.78 & 10.03 \\
\hline
\end{tabular}

having a full step at the extreme ends of an exchanger represented on a McCabe-Thiele construction. The integer number of stages for the two matches occurring in Figure4.15 is 1 and 11 respectively. The capital cost of the hybrid-based network is $\$ 512000$ which is $13.4 \%$ below the target. As noted before the comparison of any alternative synthesis method with the pinch design approach should be based on the supertargeting results. Note that there is no cost associated to the MEA in this example and hence the comparison is limited to the capital cost minimisation. Nonetheless, this reduced capital cost from the hybrid approach is followed by a $2 \%$ increase in the MSA consumption.

\subsection{Pinch-structure independent initialisations}

Various initialisation strategies were proposed in Section4.2.7 that do not rely on the pinch design-rules. This section illustrates how, during the generation of these initial solutions, the driving force plot can aid the designer to decide on the quality of the initial solution. There is currently no formalised strategy on how to initialise MINLP solvers for MENs and as a result many initial solutions are presented to an MINLP solver without the designer knowing the quality and physical difference of these solutions. A knowledge of the areas of improvement that are exposed by a driving force diagram should allow the designier to implement decisions that will drive an MINLP solver towards near-optimal solutions. The targets by themselves do not provide the guidance regarding the changes that can be made to the model to attain these solutions but merely expose the economic potential of a project.

The ultimate integration of driving force diagrams and optimisation tools will heavily rely on the availability of visual representation-tools. These tools should be implementable in a way that the designer, following an analysis of the driving force diagram, can adjust the model within the optimisation routine. In this way the designer will be involved in ensuring that solutions from which the optimal solution is to be selected are already making the best use of the available driving force and those that do not can be eliminated early from further consideration. The main aim of this section is to illustrate how the driving 
force diagram may be used to reject initialisation structures that do not lead to nearoptimal hybrid-solutions. The dephenolisation case(See Section4.3.2) will be used for this illustration.

The optimum minimum-composition-difference for this example, based on the supertarget method, is $\varepsilon=0.00025$ and the resulting $y-y *$ plot for this $\varepsilon$ is given by Figure4.16. The matches that will be generated will be superimposed on the operating line and where the match makes bad use of driving force, an alternative initial structure will be used.

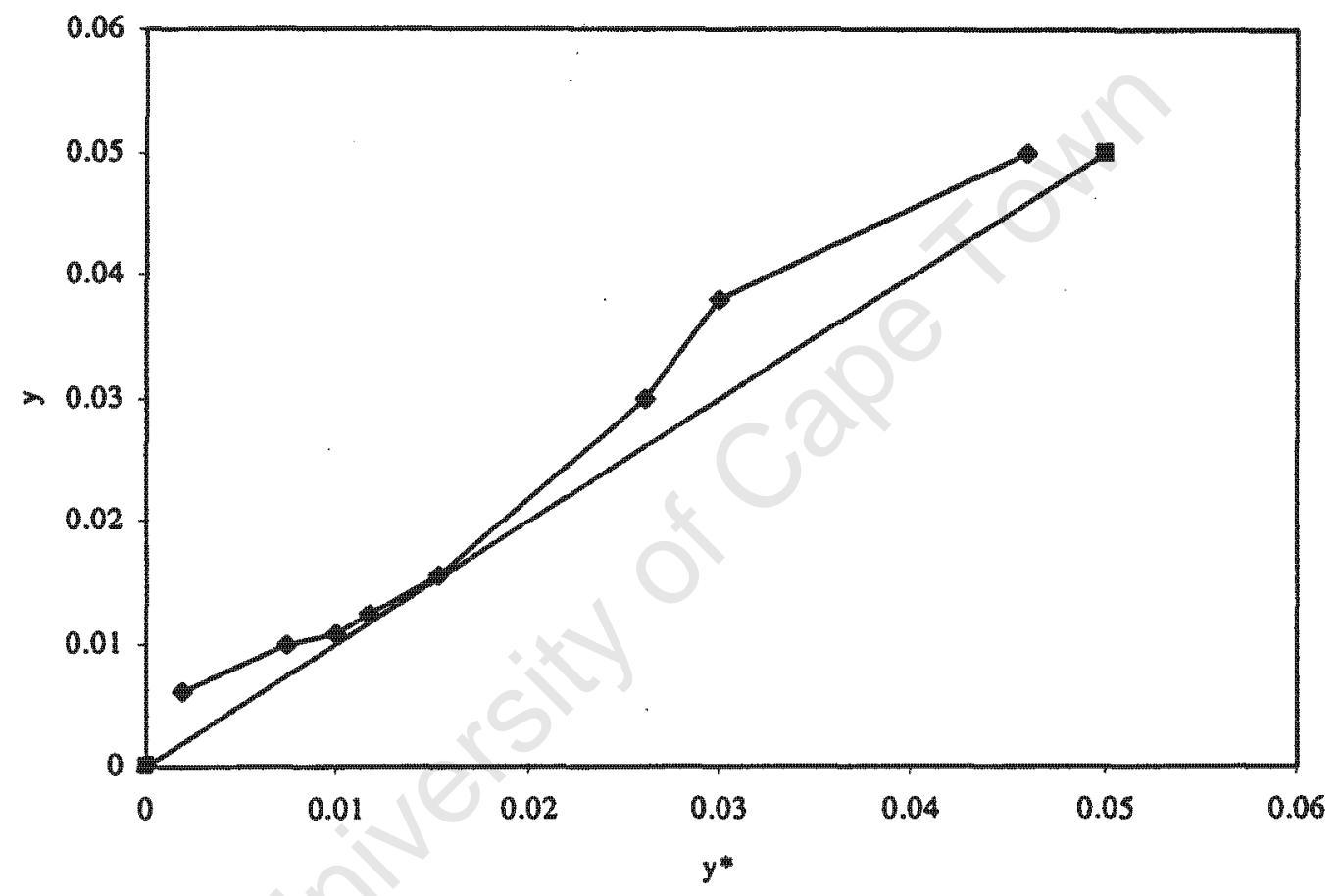

Figure 4.16: Driving force diagram for the dephenolisation example

The first initialisation strategy to consider is that of using all of the external MSA to remove the dephenolisation task as illustrated by Figure4.17. In heat exchange network synthesis this initialisation is implemented such that all the heating requirements are carried out by steam and all the cooling requirements are carried by water. The optimiser would then select the best policy in terms of how much of the available process streams and utilities to use while at the same time generating the network structure.

The main difference to be taken into account in MENS is that, unlike HENS, there is 


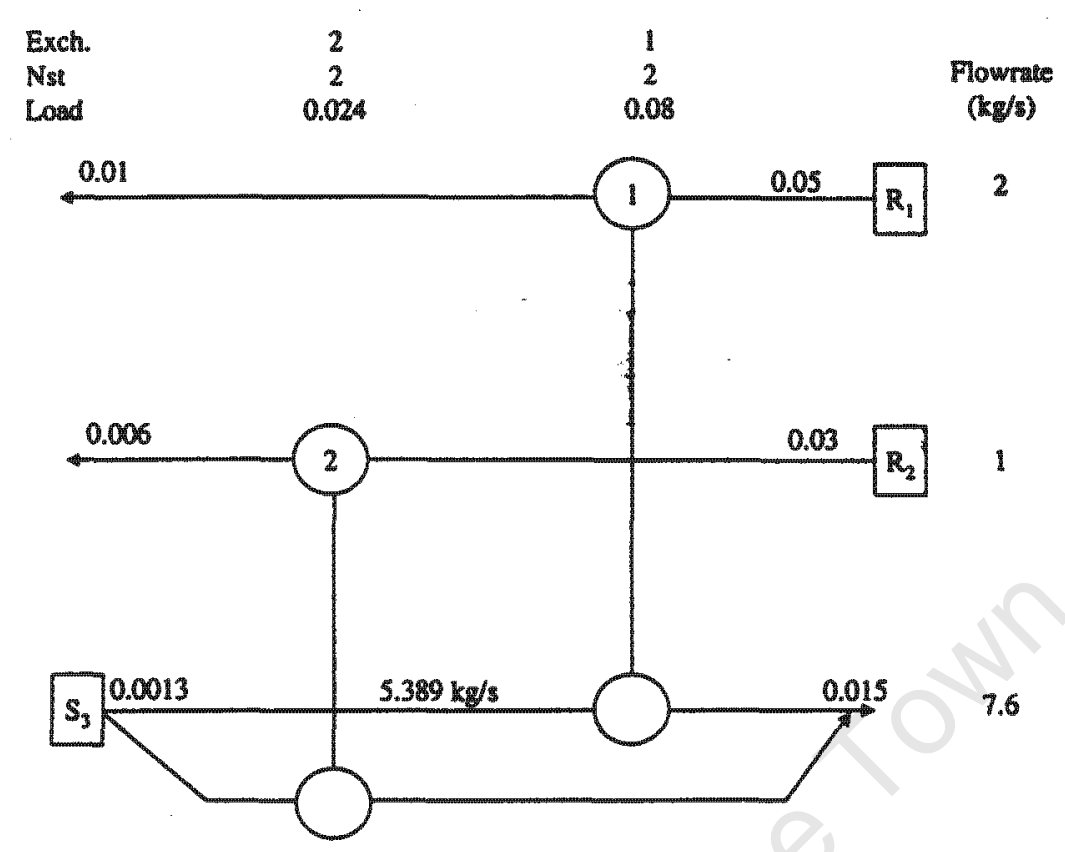

Figure 4.17: Proposed initial structure independent of the pinch structure. Capital cost = $\$ 6000$, Operating cost $=\$ 2230000$

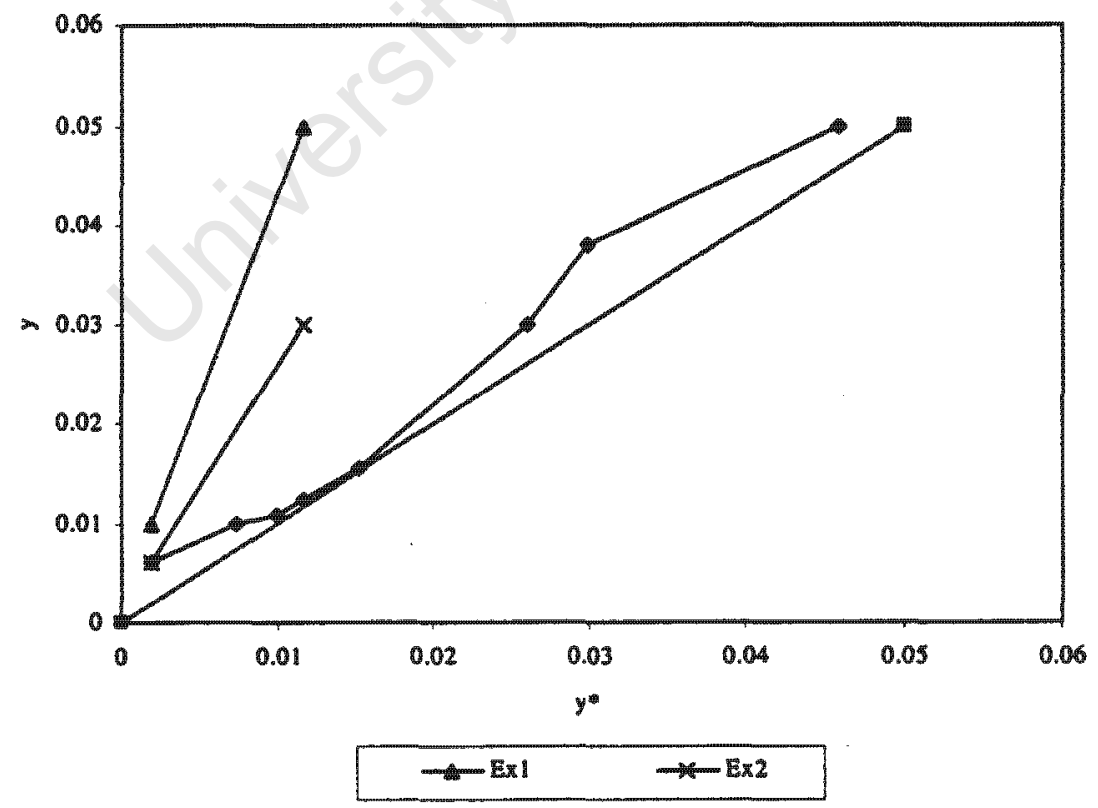

Figure 4.18: Driving force plot for the initialisation with external MSA only 
no different type of utility that is required when one is above or below the pinch point. The use of the external MSA can be modelled as a single-MSA problem analogous to the problem of minimising fresh-water usage(Wang and Smith, 1994).

The network structure in Figure4.17 features two units and is not generated according to pinch-rules. The hybrid network resulting from this initialisation is in fact similar in structure and operating conditions to Figure4.17. The solution itself is feasible and is considered by the solver to be an optimal one. The total cost for this network is $\$ 2236000$.

In Figure4.18, the exchangers 1 and 2 makes maximum use of driving force such that there remains an increased consumption of MSA. The load on either of the exchangers can be reduced by adding another exchanger whereby some of the process MSA will be used. If the load on the $S_{3}$ is kept the same and the slopes of the operating lines for exchangers 1 and 2 reduced by increasing the flowrate of $S_{3}$, the resulting network and use of driving force are still similar to Figures 4.17 and 4.18 respectively.

In the next initialisation strategy, let an additional exchanger, exchanger 3 , be used with exchanger 1 to remove the load on $R_{1}$ according to Figure4.19. The effect of splitting the load on exchanger 1 is that the inlet composition of $R_{1}$ to this exchanger will reduce to the rich outlet composition of exchanger 3 while the new exchanger takes advantage of the driving force that is available in the region above the pinch. The selection between which match (Exchanger 1 or 2) requires an additional exchanger depends on the inlet composition of the rich streams to these exchangers. The exchangers with the highest inlet composition or load are the first to have their load shared with the additional exchanger. The outlet composition of the additional exchanger is determined by the pinch-based tick-off rule which says that streams should be matched such that the match completely utilises the capacity of either of the matching streams. This however, does not have to be always the case since any choice of an outlet composition can be qualitatively evaluated by observing its effect on the driving force diagram.

For this initialisation as well, the structure of the optimum solution is similar to its initial structure. The operating conditions as shown by Figure4.19 are also the same with the network having an total annual cost of $\$ 960000$. No exchangers have been added to or removed from the initial structure and the consumption of $\mathrm{S}_{3}$ is $58 \%$ lower than in Figure4.17 since exchanger 3 accounts for the removal of most of the phenol from the region above the pinch. The driving force for the hybrid solution is given by Figure4.20 which shows how exchanger 3 has affected the use of driving force by exchanger 1 . As can 


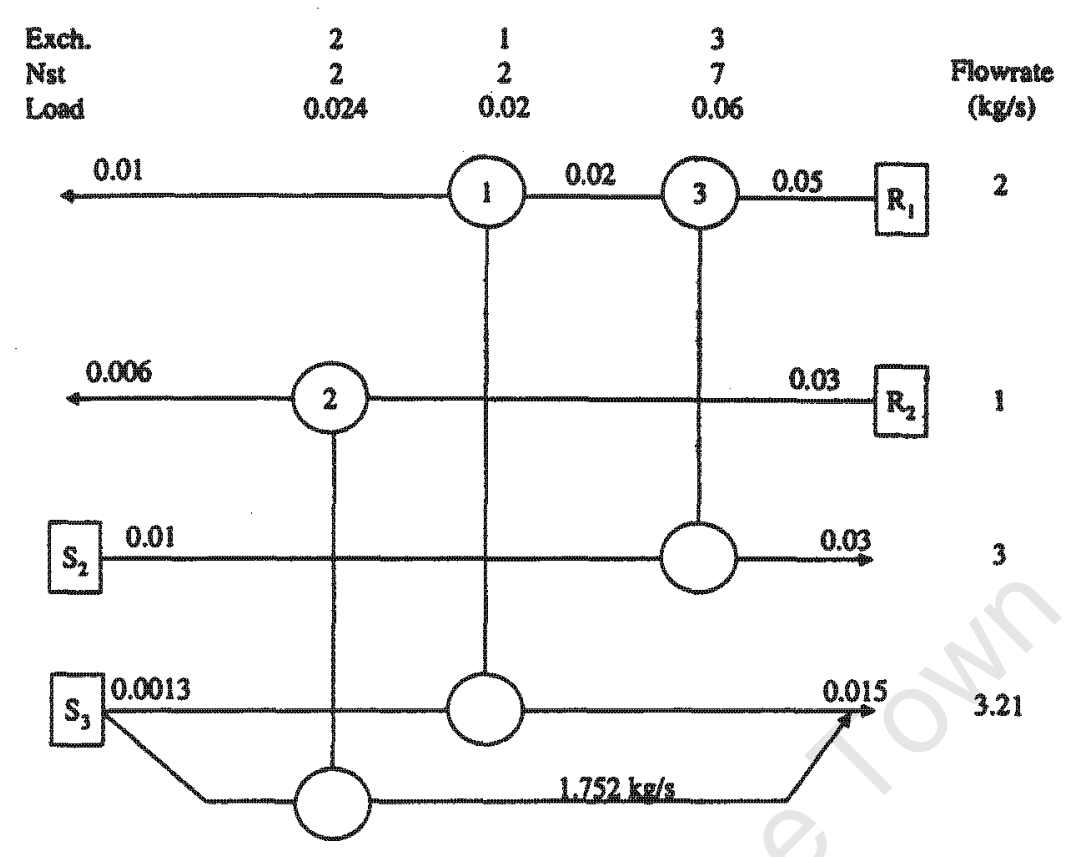

Figure 4.19: Proposed network from analysis of driving force plot to reduce outlet composition of Ex6. Capital cost $=18400 \$$, Operating cost $=\$ 942300$

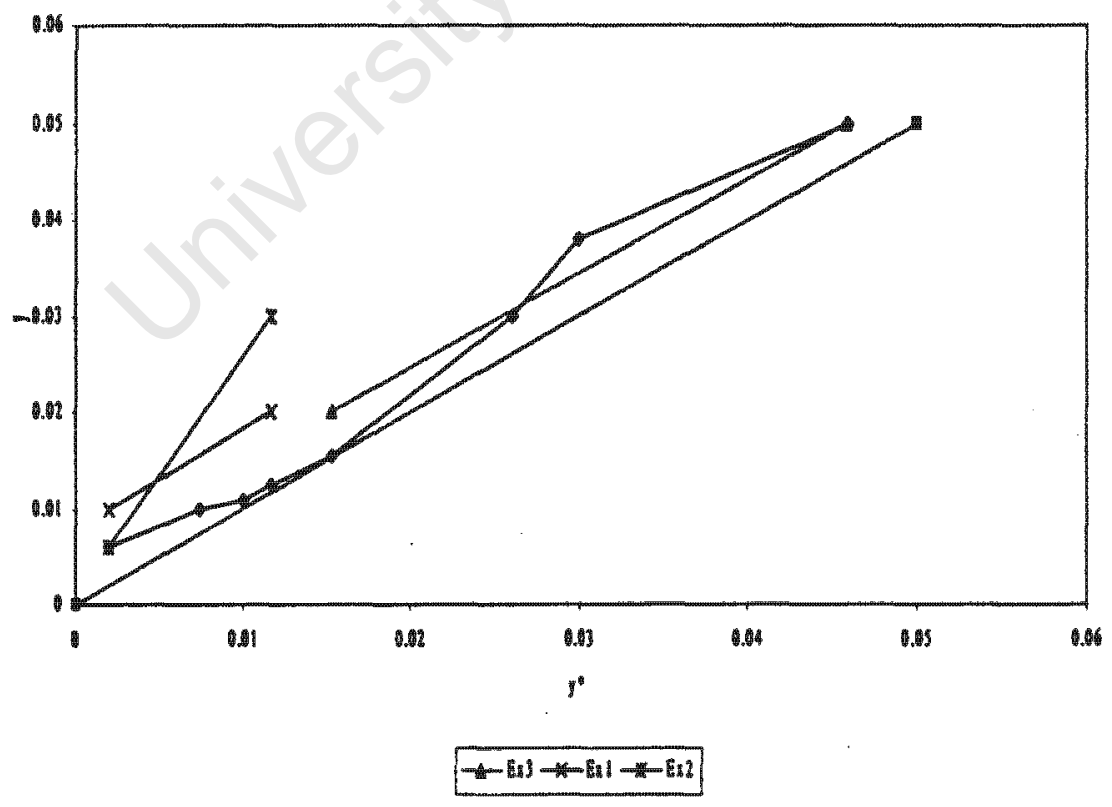

Figure 4.20: Driving force plot for the initialisation with shared loads for Ex6 
be seen exchanger 3 now has a better use of driving force as it approaches the operating line.

Many other initial structures may be systematically generated through the analysis of the evolving driving-force diagram. The solutions that were based on the external-MSA and the shared-load initialisations were similar to their initial solutions because these initial structures were simple and many variables in the MINLP model were automatically allocated initial values of zero by a pre-solve routine within the solver algorithm. It can be anticipated that as more complex initial structures are used, different MINLP solutions that take advantage of the available driving force, will be generated.

Consider the case where the load on both exchangers 1 and 2 is shared with exchangers 3 and 4 respectively and an additional exchanger 5 is allocated to treat $R_{1}$ as illustrated by Figure4.21. Exchangers 3 and 4 are aimed at reducing the outlet composition of exchangers 1 and 2. The addition of exchanger 5 is aimed at having the two exchangers ( 5 and 3 ) to trade-off their use of driving force above the pinch. Figures4.21(a)and(b) illustrates the initial and final networks resulting from these considerations.

The number of exchangers in Figures4.21(a)and(b) are the same but the interconnection has altered. There is a self-recycle of $R_{1}$ through exchanger 3 in Figure4.21(b). Recall that self recycle is a result of an exchanger that acts as a splitter and recycles a stream that is already treated (see Section4.2.2). The bypass $\mathrm{S}_{2}$ lean stream passes through this splitter, it does not exchange mass as it goes through the network and can be seen as an overall bypass. The MSA consumption in the optimum solution has decreased by $32 \%$ from its initial structure.

The hybrid solution makes good use of the driving force as can be seen in Figure4.22 resulting in the increased number of stages shown in the network structure of Figure4.21. When the total annual cost of the hybrid solution is compared to the $\$ 226000$ total cost targets that were set for the dephenolisation example in Section4.3.2, it is found to be within within $21 \%$ of the target at $\$ 272000$. In terms of the difference in the solution structure, Figure4.21 has 2 lesser number of exchangers that in Figure 4.8 which had a TAC of $\$ 235000$. Even so, both solutions have the same number of exchangers as the structures they were initialised with. Table4.4 illustrates the costs for the optimum solutions whose initial structures were independent of a pinch structure. 


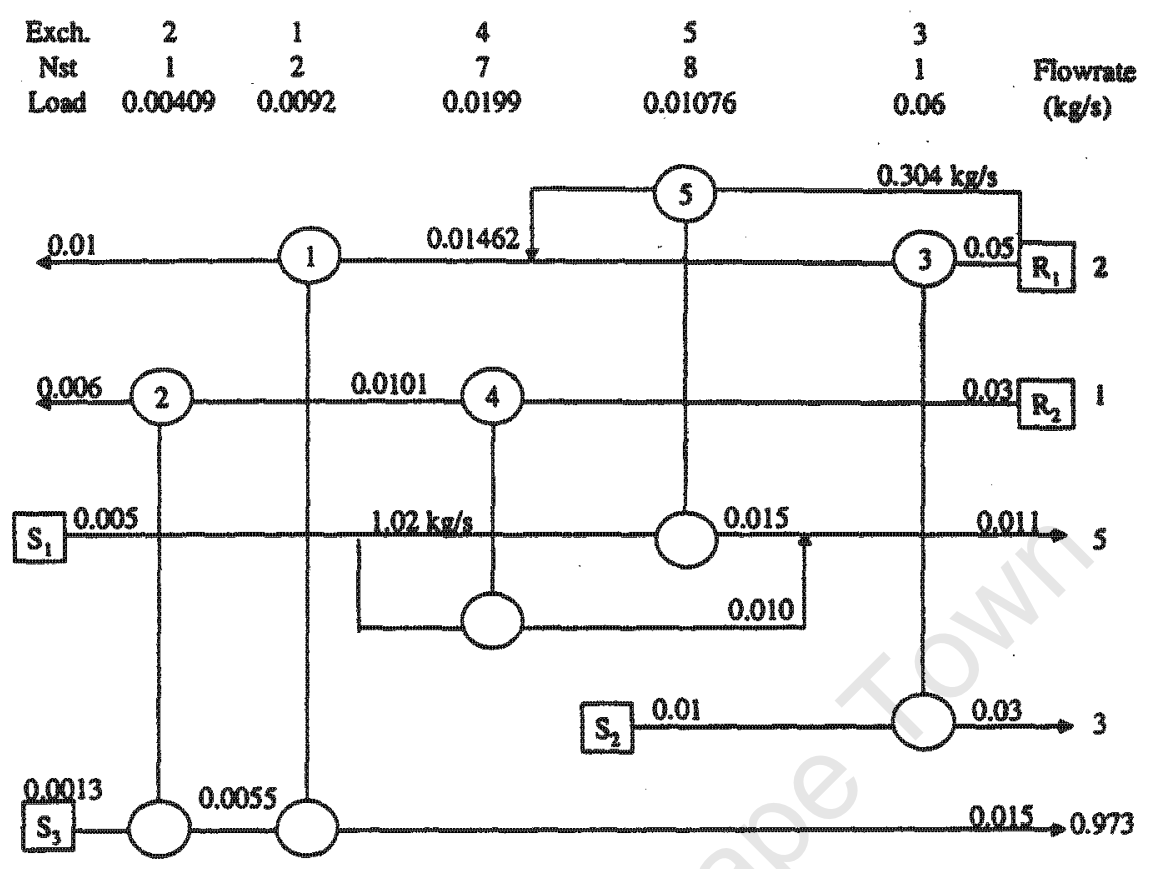

(a)

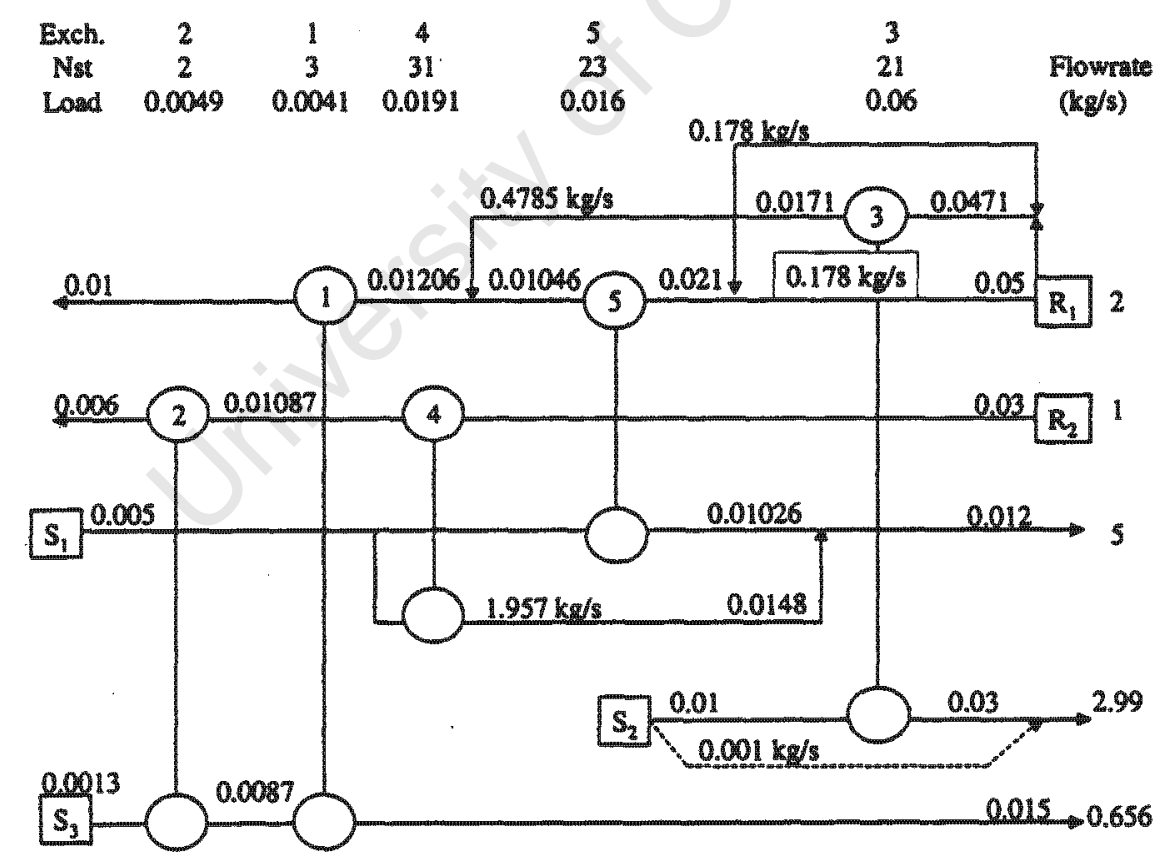

(b)

Figure 4.21: The initialisation (a) and solution (b) to the parallel-series initialisation. Capital cost $=\$ 80000$, Operating cost $=\$ 192000$ 


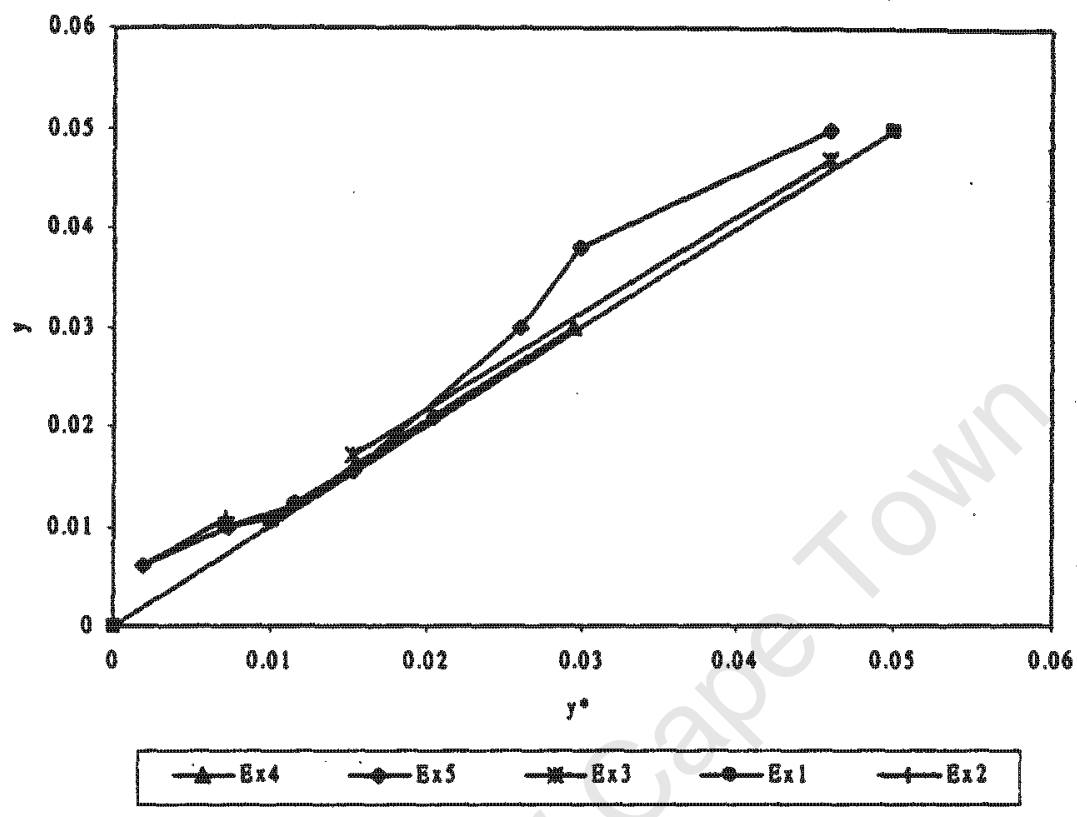

Figure 4.22: Driving force usage for the parallel-series initialisation

Table 4.4: Costs of the optimum solutions when the solver is initialised from structures that are not pinch-based

\begin{tabular}{|c|c|c|c|c|}
\hline Initialisation & TAC & OPC & CAP & Number of units \\
\hline External MSA & 2236000 & 2230000 & 6000 & 2 \\
\hline External MSA $+S_{2}$ & 960700 & 942300 & 18400 & 3 \\
\hline External MSA $+\mathrm{S}_{1}+\mathrm{S}_{2}$ & 272000 & 192000 & 80000 & 5 \\
\hline Pinch-based & 235000 & 158000 & 77000 & 7 \\
\hline
\end{tabular}




\section{Remarks}

Had the targets been used as the only insight-based tools available to the designer, the designer would have rejected this solution and yet he or she would have had no other systematic way to improve the reported solution. Both these tools, the targets and the driving force-diagram, can complement each other in a way that the driving force-tool informs about the regions in a network that show opportunities for improvement and and the targets provide the confidence in the economic quality of implementing such improvernents in the hybrid model.

The automatic allocation of initial values of zero to those variables that were not initialised by the modeller leads to optimum solutions that are not different to their initialisation structures. The designer has to trade-off the use of a structured initialisation technique where the complexity of the initial structure is gradually and systematically increased with that of a random initialisation-method where all variables in the MINLP model are allocated values that are non-zero in the hope that such initialisation will be feasible to result in a first NLP solution. The question of how to improve solutions that were initialised through a random process should make the driving-force-based approach an attractive method since it provides a framework within which the initialisation structures can have a physical meaning and can therefore be intuitively improved.

\subsection{Summary}

This section has tested a design philosophy of mass exchange networks when MINLP programming is complemented with pinch analysis tools. At the heart of the integration is the use of pinch-based total cost targets to evaluate the optimality of MINLP solutions.

The MINLP work has shown that once a model has been developed, it is easy to study the effect of changes in the problem specifications, such as stream types, exchanger types, costing models and consideration of regeneration networks. The problem of how to select the appropriate Kremser equation to size stagewise exchangers was overcome by the use of the logical operators proposed in Chapter 3. The problems with non-linear equilibrium relations were handled by the use of the McCabe-Thiele approximation method.

Experience with various examples has shown that, according to Equation4.8, the realistic number of times that streams can be allowed to match in a network is three. For the 
case-studies discussed in this chapter, when the rule of setting the number of subnetworks according to the grid-diagram analysis (see Section4.2) is used, most solutions were reported to be infeasible. Thus in most cases, limiting the number of subnetworks to 3 would yield optimal solutions.

The opportunity for the designer to interact with the MINLP design method lies in him/her being able to analyse the driving force plot for the MINLP generated matches. The driving force plot can be used to evaluate and manipulate reported solutions in an attempt to either compare the MINLP solutions or improve the initialisation of the model for the next iteration. This use of the driving force plot allows for the designer's judgement to be incorporated into the MINLP solution-search. Although the manipulations that can be made as a result of analysis by the driving force plot are not general, the availability of this option provides for a direction for the integration of intelligent visual tools with optimisation algorithms. Fragaetal.(2001) describes the implementation of an interactive visualisation procedure that is combined with a stochastic-optimisation tool for the design of heat-integrated processes.

In the next chapter, the hybrid model is applied to an industrial problem where alternative processing routes and an across-facility mass exchange network is created with minimum changes made to the topology of the existing network. 


\section{Chapter 5}

\section{Extension to an industrial problem}

Let no one ignorant of geometry enter my door

Plato $(427$ - $347 \mathrm{BC})$

This chapter aims to apply the hybrid model to an industrial problem that involves the removal of $\mathrm{H}_{2} \mathrm{~S}$ from a number of gaseous and liquid streams using alkanolamines. While this chapter intends to illustrate to industrialists, how the insight-based tools can benefit from being integrated with optimisation tools, the foregoing discussions reveal to academic researchers that most developed models make assumptions that oversimplify the problems at hand and in fact contribute to the resistance to accept these techniques. A major assumption that is made when mass exchange networks are synthesised is that an equilibrium function, describing the thermodynamic feasibility for the anticipated mass transfer, is readily available and does not vary throughout the network. In many industrial problems, this assumption is not appropriate as the conditions may be different in various exchangers.

In the industrial problem considered there are two facilities for the removal of $\mathrm{H}_{2} \mathrm{~S}$ from a refinery. Each of the facilities employs a different mass separating agent due to different purity requirements in the product streams. The constraints that the designer faces, in addition to the difficulties involved in obtaining analytical equilibrium functions that are representative of the varying process conditions, concern the geometry and operating conditions of existing units. Any improvements to the gas-sweetening facilities should achieve maximal removal or transfer of the $\mathrm{H}_{2} \mathrm{~S}$ while using as minimal an external MSA as possible. It is common that there may be requirements for an additional exchanger or 
the removal of an old exchanger. Since a network already exists, the space for the new exchanger may not be available while its placement at a location that is far away from the separation section might mean increased piping cost, delays in the implementation of the retrofit result. Pressure drops may lead also to increased costs due to a probable increase in the pumping requirements of the process. With these constraints, the designer has a choice of either ignoring these topology constraints and investigating options of interchanging the mass exchange technologies or maintaining the topology constraints.

Firstly, a brief review of topology-constrained design techniques for mass exchange networks is presented followed by a review of models that are currently used for the prediction of vapour-liquid equilibrium (VLE) data. The models considered will be limited to those applicable to the $\mathrm{H}_{2} \mathrm{~S}$-alkanolamine system. In the end, the chapter aims to elucidate the importance of integrating data prediction models, thermodynamic insights and optimisation frameworks to solve mass exchange problems. It is instructive to acknowledge that phase equilibria has been routinely modelled in the past. The discussions in this thesis therefore do not aim to develop new prediction models for VLE-data but instead, the models that are already available are used for the data prediction stage.

\subsection{Review}

\subsubsection{Topology constrained design}

Fraser and Hallale (2000) developed a retrofitting method for mass exchange networks using mass transfer pinch analysis. The approach consists of three stages: the determination of retrofit targets, the generation of a savings-investment curve for a range of minimum composition differences and the design of a network to meet the chosen targets.

Recently Fraser et al. (2001b) presented a retrofit design algorithm for MENs that are generated for pollution reduction purposes.

In all these examples, single processing facilities were considered and poor processes were intentionally selected to illustrate the retrofitting techniques. When dealing with multiple facilities the opportunities for improvement may not be necessarily obvious.

Hui and Ahmad(1994) considered the design of a heat recovery network in a process where the cost optimisation takes into account the number of interconnections between separate 
regions of a plant. The trade-off between the traditional total cost and the cost of interconnection showed that at an optimum temperature difference for a totally integrated plant, the HEN may be cheaper while the cost of interconnections could be high. On the other hand, at an optimum temperature difference for a plant with separated regions, there may be a high HEN cost but no interconnections required. The consideration for the cost of interconnections between processing facilities incorporates features of practical importance to the designer such as, for an example, plant layout.

Regarding the designer's involvement in solving problems with constrained topologies, Zhu and Asante(1999) presented a two-step hybrid retrofitting process for HENS that combines pinch analysis and optimisation. The first step - the diagnosis step - uses thermodynamic pinch analysis to select promising modifications and assesses them in terms of the impacts on implementation cost, operability and safety. The second step is a mathematical optimisation step which uses an non-linear program to minimise the cost of additional surface area employed. The diagnosis step capitalises on the judgement and experience of the designer while the NLP optimisation step accounts for the many trade-offs and constraints posed by the retrofit problem. It is this designer-optimisation interaction that the hybrid model seeks to achieve in solving industrial problems.

\subsubsection{Solubility data prediction}

Alkanoamines such as monoethanolamine, diethanolamine and mixed alkanolamines are common solvents in the removal of the acid gas contaminants, $\mathrm{H}_{2} \mathrm{~S}$ and $\mathrm{CO}_{2}$. These solvents are classified as reactive mass separating agents. The alkanolamines form weak bases which react with the acid gases in an aqueous solution. In order to predict operating characteristics and behaviour of mass exchange networks featuring these MSAs, it is necessary to have some knowledge regarding the solubility of $\mathrm{H}_{2} \mathrm{~S}$ and $\mathrm{CO}_{2}$ in the alkanolamines. A considerable amount of solubility data has been reported in the literature (seeKohl and Nielsen(1998)for examples) for different $\mathrm{H}_{2} \mathrm{~S} / \mathrm{CO}_{2}$-alkanolamine systems at various temperature and pressure conditions. The scatter of this data at similar experimental conditions makes it difficult to extrapolate the solubility results to regions where there is no experimental data and even when the extrapolation is successful, the solubility at extreme reaction regions such as at very low acid gas partial pressures, may be inaccurate due to inadequate data points. Figure5.1 illustrates this data scatter for experiments carried out at $40^{\circ} \mathrm{C}$ and an MEA concentration of $15.3 w t \%$ in water. 


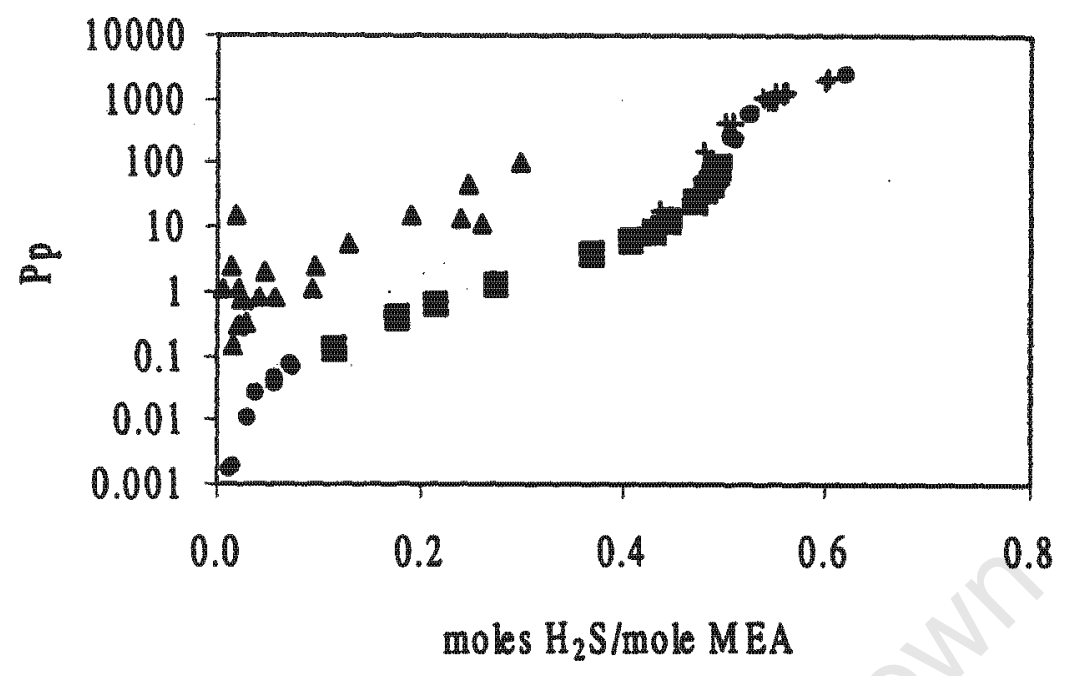

4 Kent:76 - Jones:59 - Law son:76 + Lee:74

Figure 5.1: Solubility data in literature

KentandEisenberg(1976) proposed a vapour-liquid equilibrium model (VLE) to correlate published solubility data. The model uses equilibrium constants from the literature for all but two of the reacting species in the $\mathrm{H}_{2} \mathrm{~S} / \mathrm{CO}_{2}$-alkanolamine system. The amine equilibrium constants are forced to fit published vapour pressure data for $\mathrm{H}_{2} \mathrm{~S}$-amine and $\mathrm{CO}_{2}$-amine. The model does not take into account the fugacity and activity coefficients of the reacting species and can be observed in Figure5.1, the solubility data of KentandEisenberg(1976) is out of line with that reported by JonesandClaytor(1959), LawsonandGarst(1976) and Leeetal.(1974). The equilibrium functions used in the $\mathrm{H}_{2} \mathrm{~S}$ removal examples illustrated in Section4.3.4 were proposed by Srinivas and El-Halwagi(1992) and were based on the equilibrium constants and distribution coefficients obtained from the KentandEisenberg-model. For the case of DEA, these equilibrium functions were based on an overall $\mathrm{H}_{2} \mathrm{~S}-\mathrm{DEA}$ reaction byLaletal.(1985) rather than the ionic reactions that accompany $\mathrm{H}_{2} \mathrm{~S}$-alkanolamine reactions.

Deshmuk and Mather (1981) proposed a thermodynamic model for the prediction of partial pressures of acid gases over different alkanolamine solutions. The model is based on the Debye-Huckel theory of electrolyte solutions and, unlike the KentandEisenberg-model, it takes into account the fugacity and activity coefficients of the reacting species. 
The VLE relations for the acid gas species and for the water are given by Equations5.1,5.2 and 5.3 respectively.

$$
\begin{aligned}
\phi\left(\mathrm{CO}_{2}\right) y\left(\mathrm{CO}_{2}\right) P & =H\left(\mathrm{CO}_{2}\right) \gamma\left(\mathrm{CO}_{2}\right) m\left(\mathrm{CO}_{2}\right) \\
\phi\left(\mathrm{H}_{2} S\right) y\left(\mathrm{H}_{2} \mathrm{~S}\right) P & =H\left(\mathrm{H}_{2} \mathrm{~S}\right) \gamma\left(\mathrm{H}_{2} \mathrm{~S}\right) m\left(\mathrm{H}_{2} \mathrm{~S}\right) \\
\phi_{w} y_{w} P & =\left(\mathrm{P}_{w}\right)_{V P} a_{w}
\end{aligned}
$$

where

$\phi(i) \quad$ is the fugacity coefficient of the species $i$,

$\gamma(i) \quad$ is the activity coefficient of species $i$. For water the activity coefficient $a_{w}$ also means the mole fraction,

$H(i) \quad$ is the Henry's constant for the acid gases,

$m(i) \quad$ is the molality of the species $i$,

$y(i) \quad$ is the mole fraction in the gas phase

$P \quad$ is the pressure of the system, and

$P w \quad$ is the water vapour pressure.

The chemical equilibria for the $\mathrm{H}_{2} \mathrm{~S} / \mathrm{CO}_{2}$-alkanolamine system are outlined below:

$$
\begin{aligned}
\mathrm{RNH}_{3}^{+} & =\mathrm{RNH}_{2}+\mathrm{H}^{+} \\
\mathrm{H}_{2} \mathrm{~S} & =\mathrm{HS}^{-}+\mathrm{H}^{+} \\
\mathrm{CO}_{2}+\mathrm{H}_{2} \mathrm{O} & =\mathrm{HCO}_{3}^{-}+\mathrm{H}^{+} \\
\mathrm{HCO}_{3}^{-} & =\mathrm{CO}_{3}^{2-}+\mathrm{H}^{+} \\
\mathrm{H}_{2} \mathrm{O} & =\mathrm{OH}^{-}+\mathrm{H}^{+}
\end{aligned}
$$

and each reaction equilibrium is expressed in terms of an equilibrium constant

$$
K=\prod_{i}\left(m_{i} \gamma_{i}\right)^{\nu_{i}}
$$


where $\nu_{i}$ is the stoichiometric coefficient.

The activity coefficients of the liquid phase species are determined from the Debye-Huckel law

$$
\ln \gamma i=\frac{-A z_{i}^{2} \sqrt{I}}{1+b \sqrt{I}}+2 \sum_{j} \beta_{i j} m_{j}
$$

where

$z_{i} \quad$ is the electric charge of species $i$

band $A$ are constants in the Debye-Huckel expression

$\beta_{i j} \quad$ is an interaction coefficient obtained by least squares fitting.

The interaction parameters are only defined for the dominant species pairs. The DebyeHuckel law takes into account both the electrostatic forces (through the first term) and the Van Der Waals forces (through the $\beta_{i j}$ term).

In addition to Henry's law and relations for the equilibrium constants, the equilibrium molalities, $m(i)$, must also satisfy charge balance and mass balances for the reacting species. The steps that are followed in generating the equilibrium function are as follows:

1. Supply the loading or partial pressures for the acid-gases, the temperature at which the separation is expected to occur and the stoichiometric concentration or weight percentage of the alkanolamine.

2. Obtain from equations of state the activity and fugacity coefficients of the reacting species and from Equation5.4the interaction parameters of the dominant species pairs.

3. Obtain the molalities of the species from the mass balances and the remaining set of equations.

4. Convert the molalities of the acid-gases to liquid mole fractions according to

$$
x_{\mathrm{H}_{2} S}=\frac{m_{\mathrm{H}_{2} S}}{m_{\text {amine }}+m_{\mathrm{H}_{2} \mathrm{O}}+m_{\mathrm{H}_{2} S}+m_{\mathrm{CO}_{2}}}
$$

- similarly for $\mathrm{CO}_{2}$. Note that the molalities include both physically and chemically bound components of the amine, water and acid-gases. 
5. Based on the operating pressures of the streams in the problem, convert the partial pressures of the acid-gases to mole fractions in the gas phase $y_{H_{2} S}$ and $y_{\mathrm{CO}_{3}}$.

The use of partial pressures ensures that the composition of the other species in the system is properly accounted for as the pressure of the system changes.

The task of generating equilibrium functions is important during retrofitting, if it is desired to test the performance of alternative solvents without having to undertake expensive and intensive laboratory work. Another reason is that since retrofitting deals with plants that are already existing, some information may be proprietary, thus necessitating the consideration for data prediction. Data already published may be insufficient or not dealing with the current plant operating conditions. It is also inappropriate to use VLE data that have been predicted at operating conditions other than the ones in question. The conditions in question may have been specified the way they are because of purity specifications, safety considerations when larger pressures are used and capital cost considerations to avoid cooling or heating of streams that enter the mass exchange network.

\subsection{The case study}

\subsubsection{Problem description}

In a petroleum refinery it is desired to remove $\mathrm{H}_{2} \mathrm{~S}$ from light petroleum gas and liquid streams. Hydrogen sulphide is corrosive and toxic to both animals and vegetation and has to be reduced to specified levels in the refinery product streams. Monoethanolamine (MEA) and diethanolamine (DEA) are the current solvents for the separation process. The MEA is responsible for the removal of $H_{2} S$ from $5 H_{2} S$-laden streams $R_{1}, R_{2}, R_{3}, R_{4}$ and $R_{5}$ at different temperature and pressure conditions. It should be noted that $R_{2}$ and $R_{3}$ are liquid streams, and the rest are gaseous. The DEA is used in the removal of $\mathrm{H}_{2} \mathrm{~S}$ from another $\mathrm{H}_{2} \mathrm{~S}$-rich gas stream, $\mathrm{R}_{6}$. Figure5.2 illustrates the current plant configuration for the MEA system with packed-column mass exchangers. The DEA flowsheet, Figure5.3, is similar to the MEA flowsheet with one unit and operating conditions that are similar to $R_{2}$ and $R_{3}$.

Figure5.4illustrates the different regions and alternatives that can be explored in the case study to achieve a processing facility that is cheaper. $N 1$ (MEA) and $N 2$ (DEA) represent the current $\mathrm{H}_{2} \mathrm{~S}$ removal systems of the refinery. $N 3$ concerns the option of using DEA 


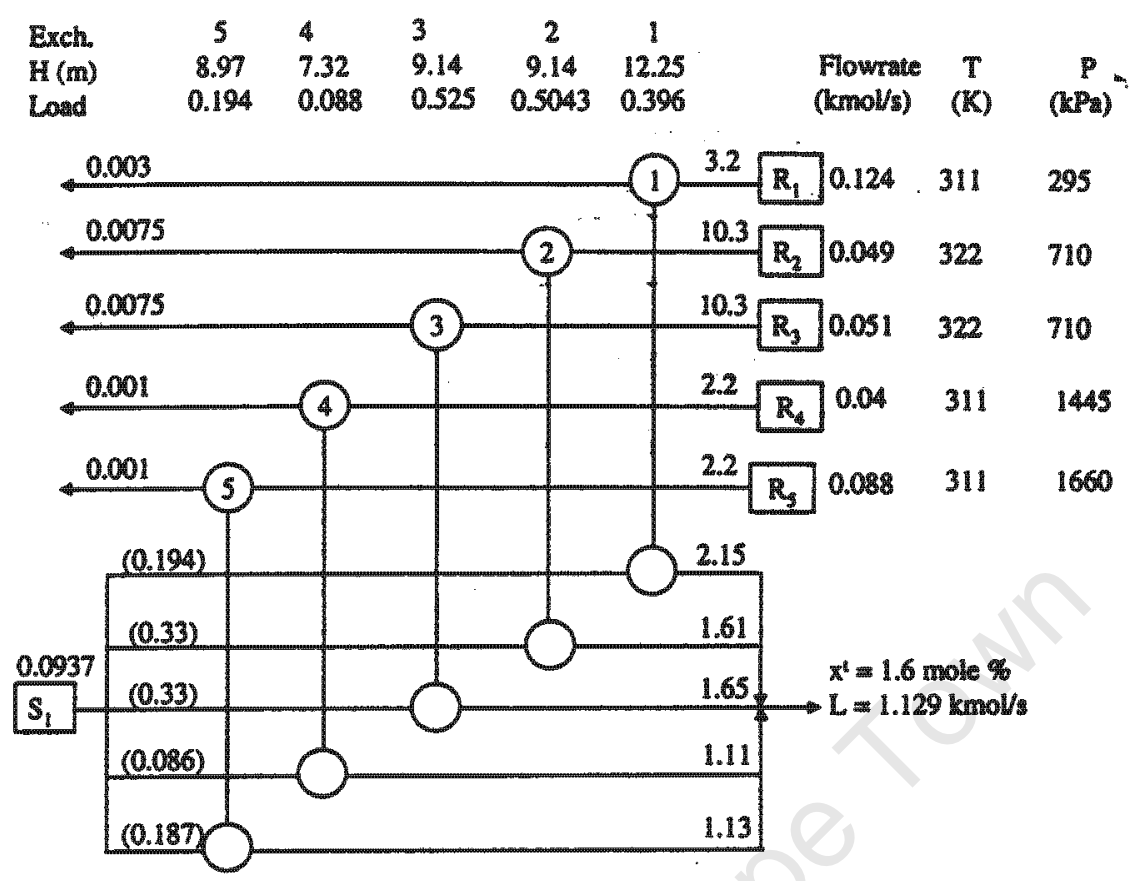

Figure 5.2: Refinery flowsheet for the $\mathrm{H}_{2} \mathrm{~S}-\mathrm{MEA}$ section ( $\mathrm{N10}$ )

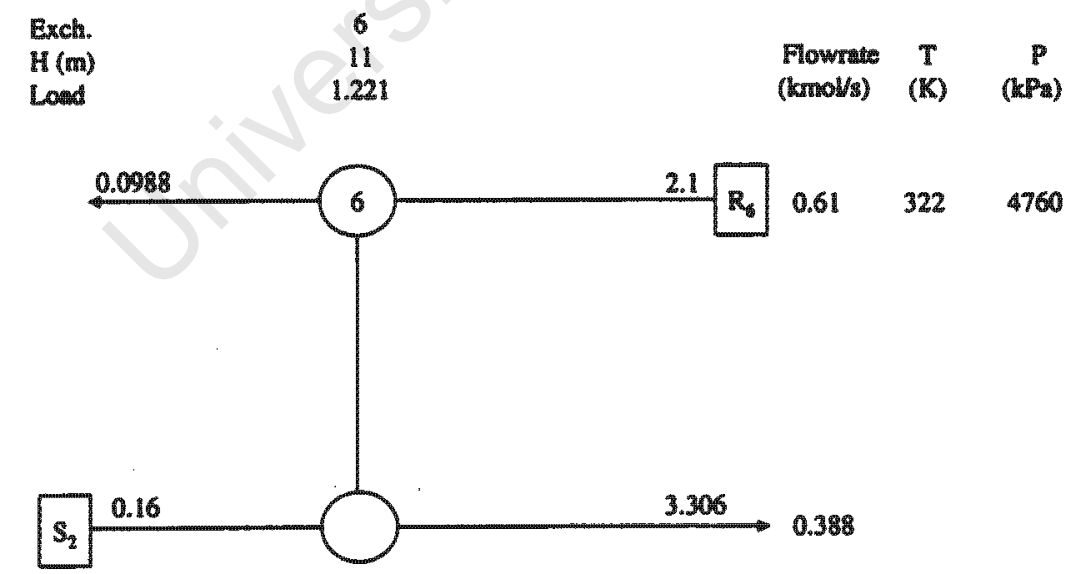

Figure 5.3: Refinery flowsheet for the $\mathrm{H}_{2} \mathrm{~S}-\mathrm{DEA}$ section (N2o) 

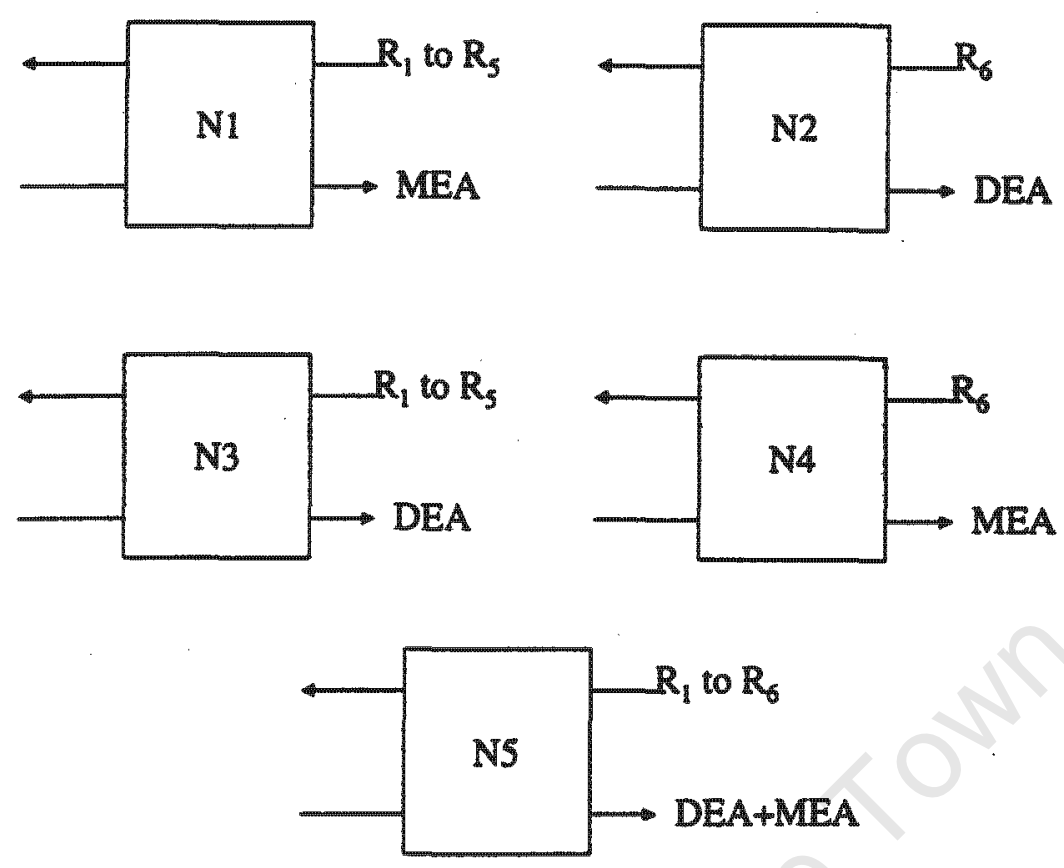

Figure 5.4: Options for the use of MSA in the $\mathrm{H}_{2} \mathrm{~S}$ removal case study

to remove the $\mathrm{H}_{2} \mathrm{~S}$ in $\mathrm{N1}$ while $N 4$ concerns the removal of $\mathrm{H}_{2} \mathrm{~S}$ with MEA in $N 2 . N 5$ represents a totally integrated mass exchange network where the MEA and DEA are simultaneously allowed to match with any of the 6 rich streams. Detailed specifications for this problem are given in AppendixD.

\subsubsection{Equilibrium function generation}

The existence of the rich streams at different temperatures and pressures poses an interesting point in the use of a single equilibrium function. Previously, problems that featured a single MSA had a single equilibrium function allocated to them. In this study, although there is a single MSA for the $\mathrm{H}_{2} \mathrm{~S}-\mathrm{MEA}$ system, the fact that the exchangers in the current plant are at different operating conditions, means that a different equilibrium function should be generated for each rich stream. Each of the rich streams is treated at a different temperature and pressure in the exchangers. The rich streams are introduced to the exchangers at a temperature higher than the absorption temperature. The equilibrium curves are convex power functions determined at the rich stream outlet conditions (assumed to be equivalent to the absorption temperature and pressure). The outlet conditions were chosen because at these conditions, the partial pressure of $\mathrm{H}_{2} \mathrm{~S}$ in the gas stream is very 
low. An MSA should therefore be able to remove $\mathrm{H}_{2} \mathrm{~S}$ at these critical conditions. Table5.1 summarises the equilibrium functions at these outlet conditions. These functions are the result of a best fit to the vapour liquid equilibrium data generated from the DeshmukMather model discussed in Section5.1. The stream pressure affects the coefficient of the equilibrium function and the temperature affects the exponent.

Table 5.1: Equilibrium functions for the MEA system

\begin{tabular}{|c|c|c|c|c|}
\hline $\mathrm{T}(\mathrm{K})$ & $\mathrm{P}(\mathrm{kPa})$ & $\mathrm{H}_{2} \mathrm{~S}-\mathrm{MEA}$ & $\mathrm{H}_{2} \mathrm{~S}-\mathrm{DEA}$ & Stream \\
\hline \hline 311.15 & 295 & $y=0.1773 x^{1.8039}$ & $y=2.6341 x^{2.1847}$ & $\mathrm{R}_{2}$ \\
\hline 322.15 & 710 & $y=0.1038 x^{2.412}$ & $y=1.035 x^{2.0584}$ & $\mathrm{R}_{2}$ and $\mathrm{R}_{3}$ \\
\hline 311.15 & 1445 & $y=0.0362 x^{1.8039}$ & $y=0.5378 x^{2.1487}$ & $\mathrm{R}_{4}$ \\
\hline 311.15 & 1660 & $y=0.0315 x^{1.8039}$ & $y=0.4681 x^{2.1487}$ & $\mathrm{R}_{5}$ \\
\hline 322.15 & 4760 & $y=0.01549 x^{2.412}$ & $y=0.1545 x^{2.0584}$ & $\mathrm{R}_{6}$ \\
\hline
\end{tabular}

The MEA equilibrium relations are based on the assumption that there is no $\mathrm{CO}_{2}$ in the system. This assumption is based on the observation that at the equilibrium-end or the exit-end of the rich streams, there is very little or no $\mathrm{CO}_{2}$ in the rich streams. This is another reason why the outlet conditions have been selected for the generation of equilibrium data.

The $\mathrm{CO}_{2}$ from the retrofit problem is present in the MEA at a content of $0.59 \mathrm{~mole} \%$ and in the $R_{2}$ and $R_{3}$ streams that contain $\mathrm{CO}_{2}$ at a content of $3 \mathrm{~mole} \%$. When the effect of $\mathrm{CO}_{2}$ is taken into account, the solubility-partial pressure curve of the $\mathrm{H}_{2} \mathrm{~S}-\mathrm{MEA}$ system may look like Figure5.5 which shows that the presence of $\mathrm{CO}_{2}$ during $\mathrm{H} 2 \mathrm{~S}$ removal will hinder the ability of the rich streams to attain high purity which is denoted by very low partial pressure values. Details of the determination of the equilibrium relations are also given in AppendixD.

\subsection{Analysis of various MEN options}

The discussion in this section refers to the results illustrated in Figure5.6 and tabulated in Table5.2. The design options for the $\mathrm{H}_{2} \mathrm{~S}$ removal process refer to the option of optimising the MSA networks separately $(N 1+N 2)$, with the DEA replaced by the MEA $(N 10+N 4)$, with the MEA replaced by the DEA $(N 20+N 3)$, with the MSAs swapped around $(N 3+N 4)$ and the option of simultaneously optimising the two MSA networks $(N 5)$. 


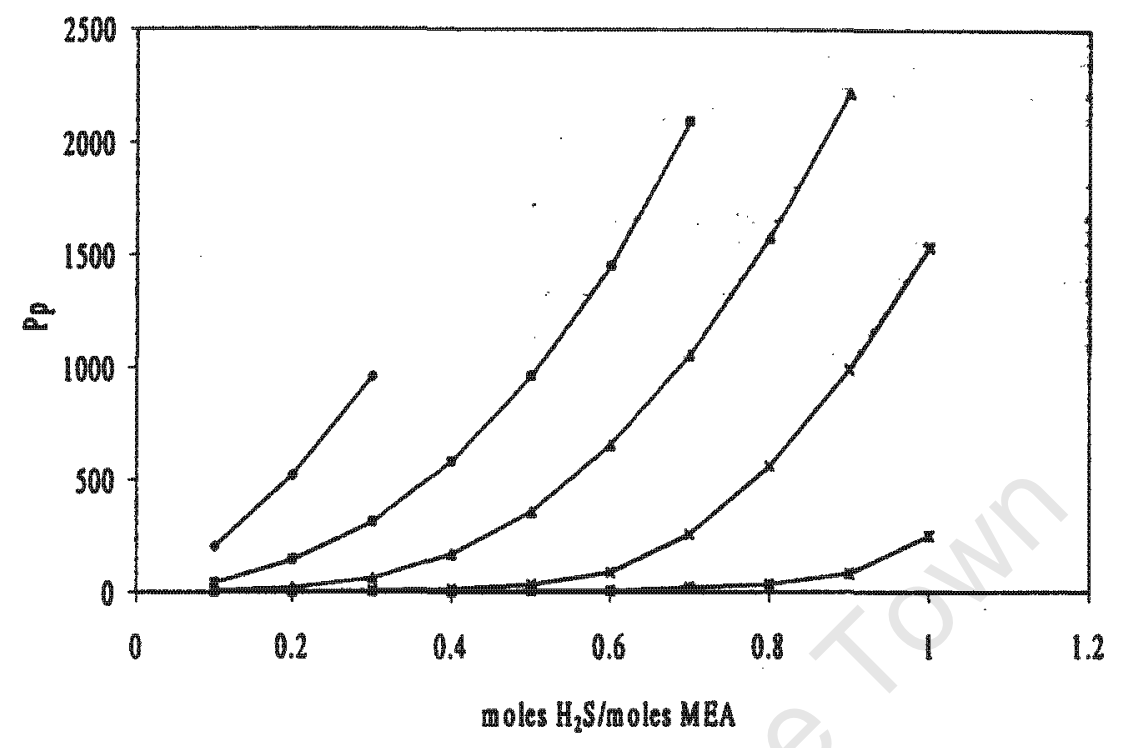

$-P p .0 .9-P p .0 .6-P p .0 .4-P p-0.2-P p .0$

Figure 5.5: Effect of $\mathrm{CO}_{2}$ on the solubility of $\mathrm{H}_{2} \mathrm{~S}$ in MEA

Table 5.2: Summary of results for the gas sweetening case study.

( ${ }^{*} 10$ and $\mathrm{N} 20$ cost used as a basis for the calculation of \% savings or investment)

\begin{tabular}{|c|c|c|c|c|}
\hline Network & $\begin{array}{c}\text { Additional CAP } \\
k \$\end{array}$ & $\begin{array}{c}\text { OPC } \\
k \$\end{array}$ & $\begin{array}{c}\text { MEA } \\
\left(10^{3} \mathrm{~m}^{3} / h r\right)\end{array}$ & $\begin{array}{c}\text { DEA } \\
\left(10^{3} \mathrm{~m}^{3} / h r\right)\end{array}$ \\
\hline \hline$N 10+N 20^{*}$ & 329 & 2656 & 83.9 & 29.8 \\
\hline$N 10+N 2$ & 475 & 2629 & 83.9 & 28.9 \\
\hline$N 1+N 20$ & 498 & 1391 & 24.1 & 29.8 \\
\hline$N 1+N 2$ & 644 & 1364 & 24.1 & 28.9 \\
\hline$N 10+N 4$ & 352 & 2412 & 111.8 & 0 \\
\hline$N 20+N 3$ & 638 & 2995 & 0 & 104.8 \\
\hline$N 3+N 4$ & 661 & 2751 & 27.9 & 75 \\
\hline$N 5$ & 530 & 776 & 35.4 & 0 \\
\hline$N 5+D E A$ & 655 & 1880 & 27.4 & 45.3 \\
\hline
\end{tabular}




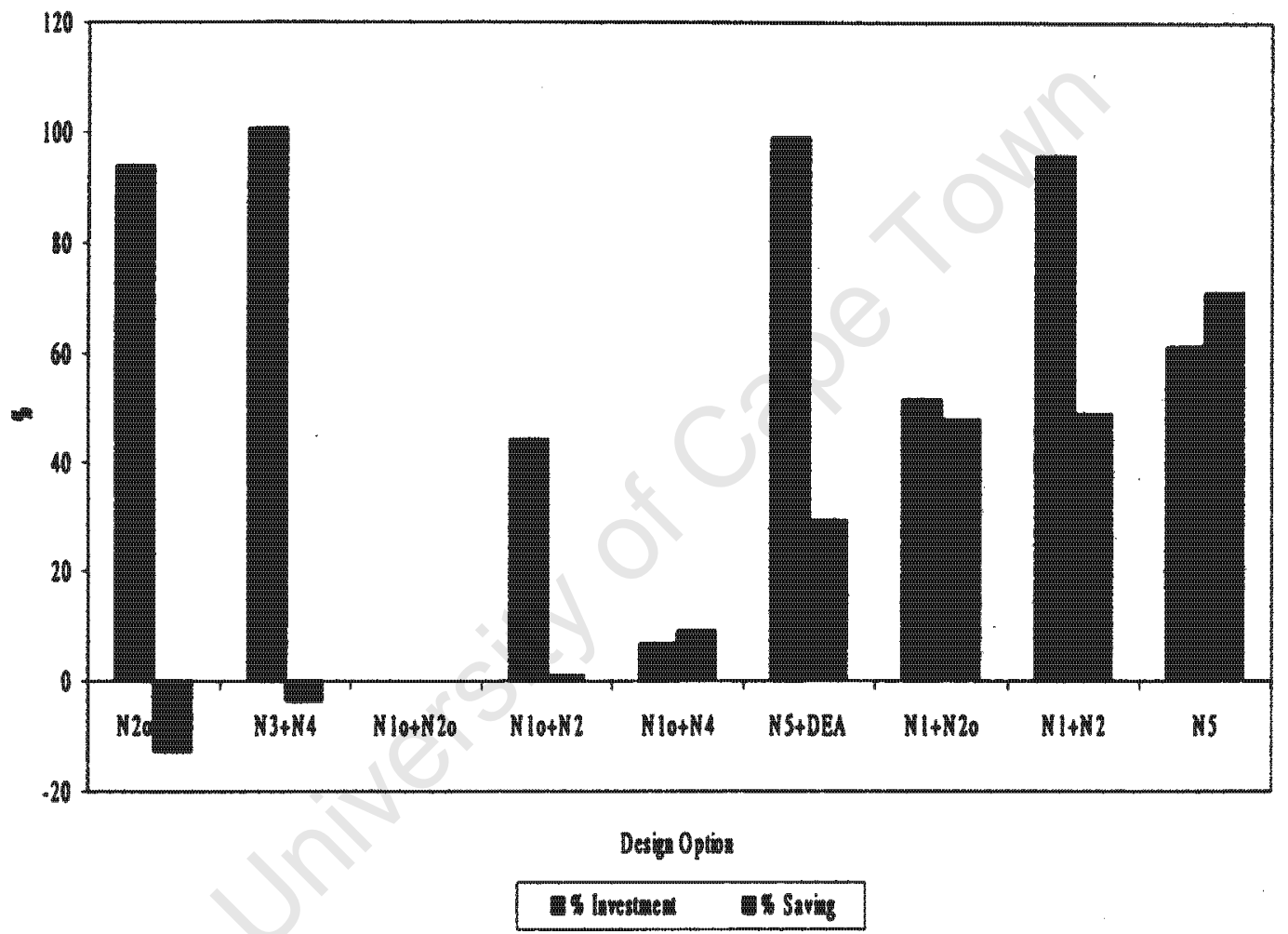

Figure 5.6: Savings and investment distribution for selected design options 


\subsubsection{Maintaining the current facility layout}

The current plant status is such that the alkanoamines, MEA and DEA, are used separately in their respective regions. That is, the MEA is used in N1 and the DEA in N2 only. The hybrid model is employed to optimise these regions separately. The structure of the hybrid network for both MSA-facilities is not different to the original plant layout. The observable changes are in the exchanger sizes and the MSA circulation rates.

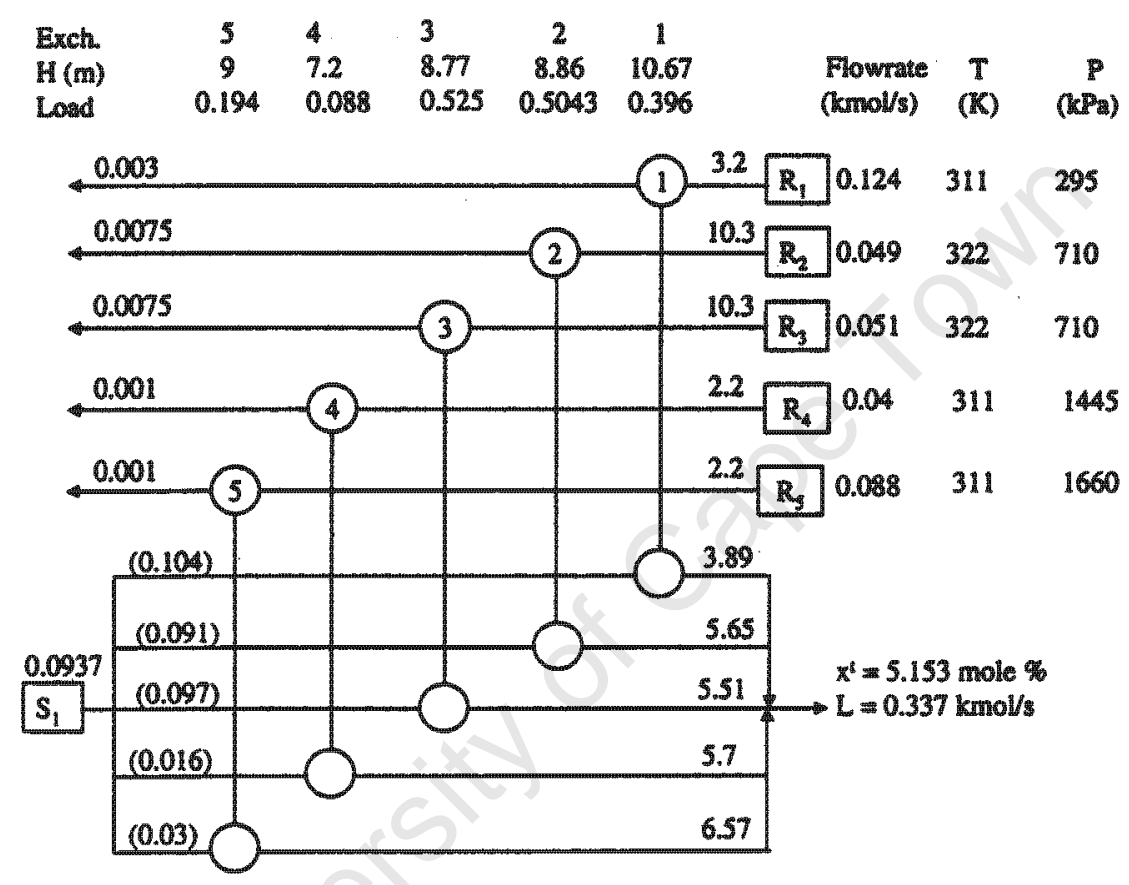

Figure 5.7: New MEA flowsheet (N1)

MEA consumption in the $N 1$ network (Figure5.7) has reduced from $1.129 \mathrm{kmol} / \mathrm{s}$ (equivalent to $83.9 \times 10^{3} \mathrm{~m}^{3} . h r^{-1}$ ) to $0.337 \mathrm{kmol} / \mathrm{s}$ (equivalent to $24.1 \times 10^{3} \mathrm{~m}^{3} . h r^{-1}$ ). In the DEA system, Figure5.8, the DEA consumption has decreased by $3.1 \%$ from $29.810^{3} \mathrm{~m}^{3} \cdot h r^{-1}$ to $28.9 \times 10^{3} m^{3} . h r^{-1}$.

The new network, referred to as $N 1+N 2$ is realisable subject to an additional capital investment of $\$ 315000$ mostly incurred by the DEA system. The inlet composition of the DEA system $(0.16$ mole\%) is very high and it leads to a very small composition difference that requires the use of a large exchanger, hence the $57.4 \mathrm{~m}$ high exchanger in Figure5.8. For the purposes of this study it will be assumed that the sizes of the exchangers are practical enough for the implementation of the proposed design-options. 


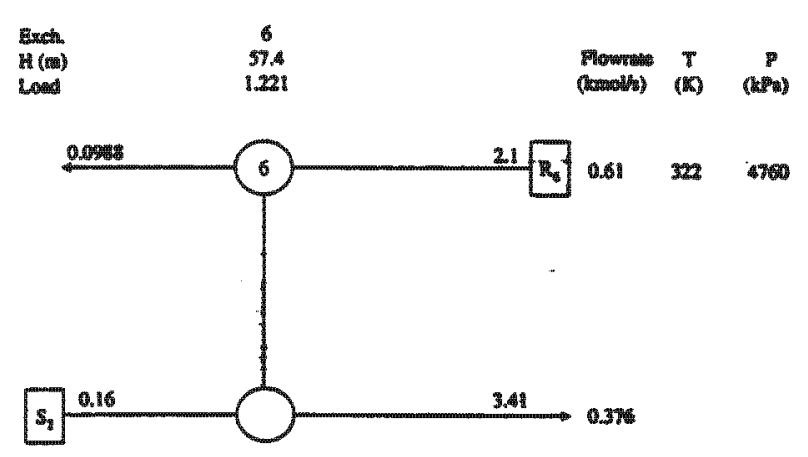

Figure 5.8: New DEA flowsheet (N2)

\subsubsection{Interchanging the MSAs}

The MSAs for $N 1$ and $N 2$ are swapped to see the economic effect of using an alternative MSA for improving the trade-off between the type of MSA used, its consumption and the additional investment cost required by the resulting network. The study into the replacement of MSAs aims to model the alternative process routes for the removal of $\mathrm{H}_{2} \mathrm{~S}$ from refinery streams using either MEA or DEA and sometimes, both. The cost of some alkanoamines are given in Table5.3(?). The annualised cost of the $20 w t \%$ MEA and $25 w t \%$ DEA used in this study is $\$ 159.5 \times 10^{6} /(\mathrm{kmol} / \mathrm{s})$ and $\$ 220 \times 10^{6} /(\mathrm{kmol} / \mathrm{s})$ respectively. The annual cost is based on the MEA and DEA usage being $1 \%$ of the circulation rates.

It is evident from the cost of the MSAs that a small reduction in MSA consumption will bring about large savings in the operating cost. The trade-off between the MSAs is that of the cost of the MSA and capacity of the MSA to adsorb $\mathrm{H}_{2} \mathrm{~S}$. There is no reason why the other alkanoamines can not be tested in this industrial case study. The focus on the DEA and the MEA is based on the fact that these are the alkanoamines that are currently used on the studied $\mathrm{H}_{2} \mathrm{~S}$-removal plant.

The N3 and N4 structures are given by Figures5.9(a) and 5.9(b) respectively, and they are similar to Figures5.7 and 5.8. Note that the inlet composition of the DEA into the MEA system in Figure5.9(a) has been deliberately reduced to 0.04 mole\% since it is thermodynamically infeasible to have a 0.16 mole\% DEA removing the $\mathrm{H}_{2} \mathrm{~S}$ to the purity-levels required by the rich streams. The realisation of such low composition will strongly depend 


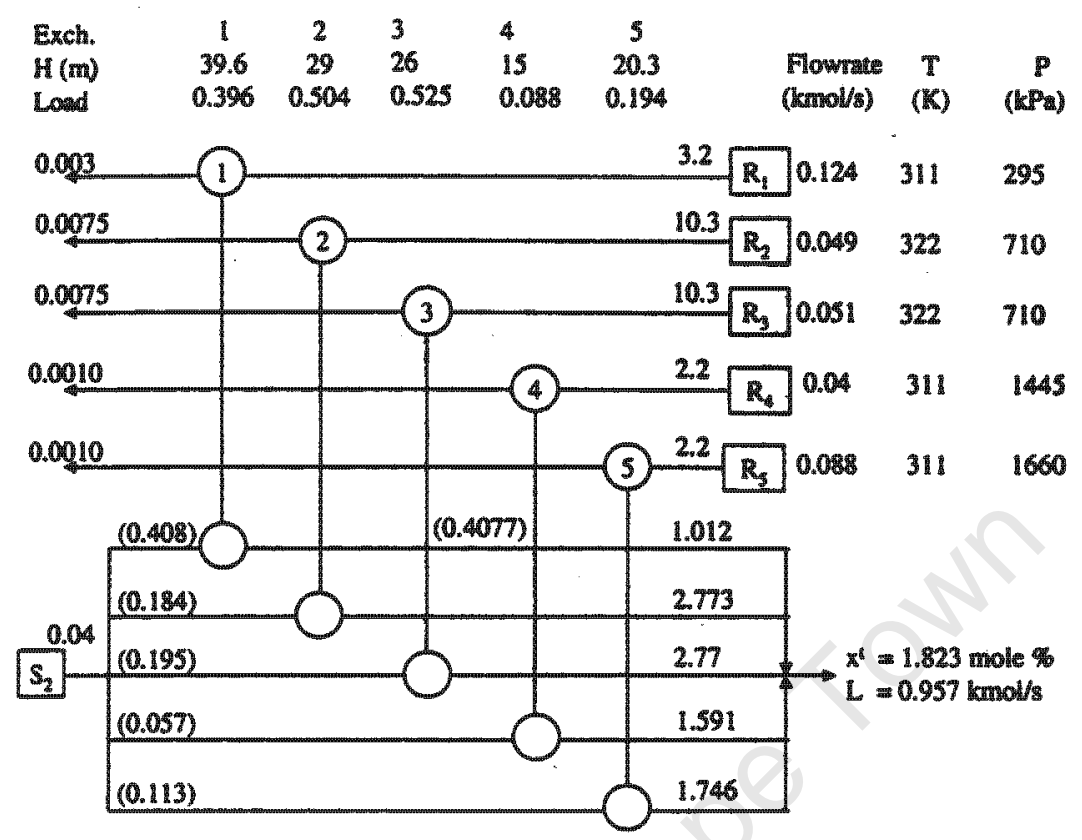

(a)

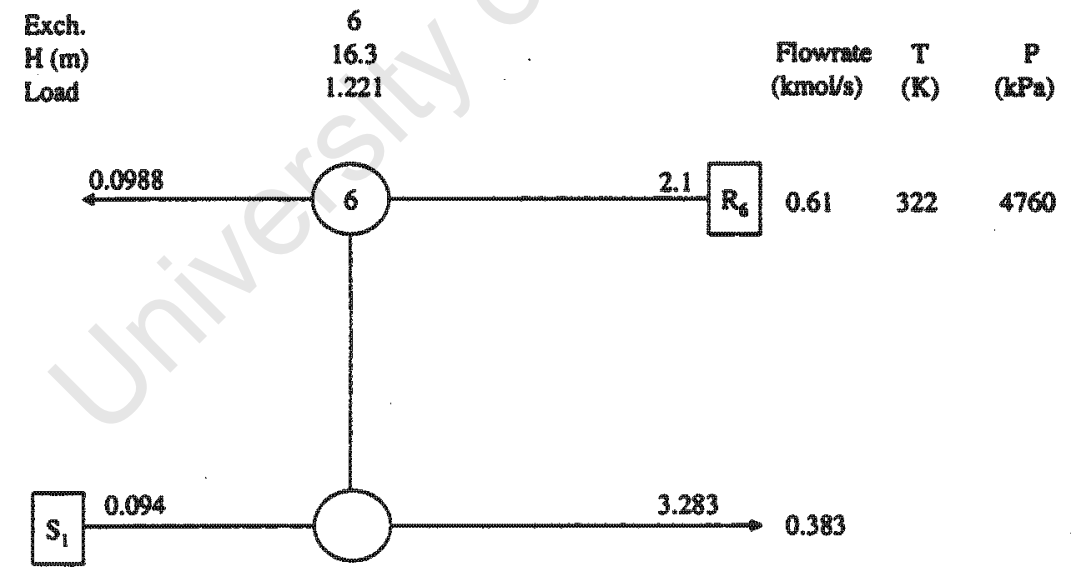

(b)

Figure 5.9: Networks illustrating (a) DEA replacing MEA (N3) and (b) MEA replacing DEA $(N 4)$ 
Table 5.3: Prices for bulk sales of alkanoamines(?)

\begin{tabular}{|c|c|}
\hline Alkanolamine & $\begin{array}{c}\text { Approximate cost } \\
(\$ / \mathrm{kg})\end{array}$ \\
\hline Monoethanolamine & 1.3 \\
\hline Diethanolamine & 1.323 \\
\hline Triethanolamine & 1.345 \\
\hline Methyl-diethanolamine & 3.086 \\
\hline Diglycolamine & 2.05 \\
\hline
\end{tabular}

on the ability of the regeneration-unit to reduce the $\mathrm{H}_{2} \mathrm{~S}$ level in the lean DEA stream to 0.04 mole\%.

Assuming that it is possible to reduce the $\mathrm{H}_{2} \mathrm{~S}$ levels in the lean DEA stream, $\mathrm{S}_{2}$ to 0.04 mole $\%$, the flowrate requirements for the DEA in Figure $5.9(\mathrm{a})$ is $75 \times 10^{3} \mathrm{~m}^{3} / \mathrm{hr}$. The additional capital investment required to use the DEA in $N 3$ is a large amount of $\$ 309000$. This network corresponds to option $N 20-N 3$. This option of using DEA in the MEA facility leads to very high capital investment and operating cost requirements. Although the amount of MSA in circulation is lower $\left(75 \times 10^{3} \mathrm{~m}^{3} / \mathrm{hr}\right)$ in $N 3$ when the DEA is used than when MEA is used, the high cost of DEA has contributed to the increased operating costs while the height requirements of matches $1,2,3$ and 5 in Figures5.9(a) led to the high capital investment.

As for the $N 4$ network, Figure5.9(b), the MEA requirement for this system is $0.383 \mathrm{kmol} / \mathrm{s}$ (equivalent to $27.9 \times 10^{3} \mathrm{~m}^{3} / \mathrm{hr}$ ) which is realisable subject to an additional capital investment of $\$ 23000$. This option $(N 10+N 4)$ is very attractive due to the $28.5 \%$ reduction in MSA cost compared to $N 2 o$. This reduction in operating cost is due to the elimination of DEA in $N 2 o$ or $N 2$. The total additional-investment that is required for completely swapping the MSAs is $\$ 315000$.

\subsubsection{Total Integration}

The network resulting from simultaneously optimising the $N 1$ and $N 2$ networks, Figure5.10 shows that only the MEA is required to remove the $\mathrm{H}_{2} \mathrm{~S}$ from the rich streams. The MEA required in this network is $35.4 \times 10^{3} \mathrm{~m}^{3} / \mathrm{hr}$ and corresponds to $74 \%$ savings in operating cost and an additional investment of $\$ 201000$.

The major structural modification to this network will be to exchanger 6 since its height 


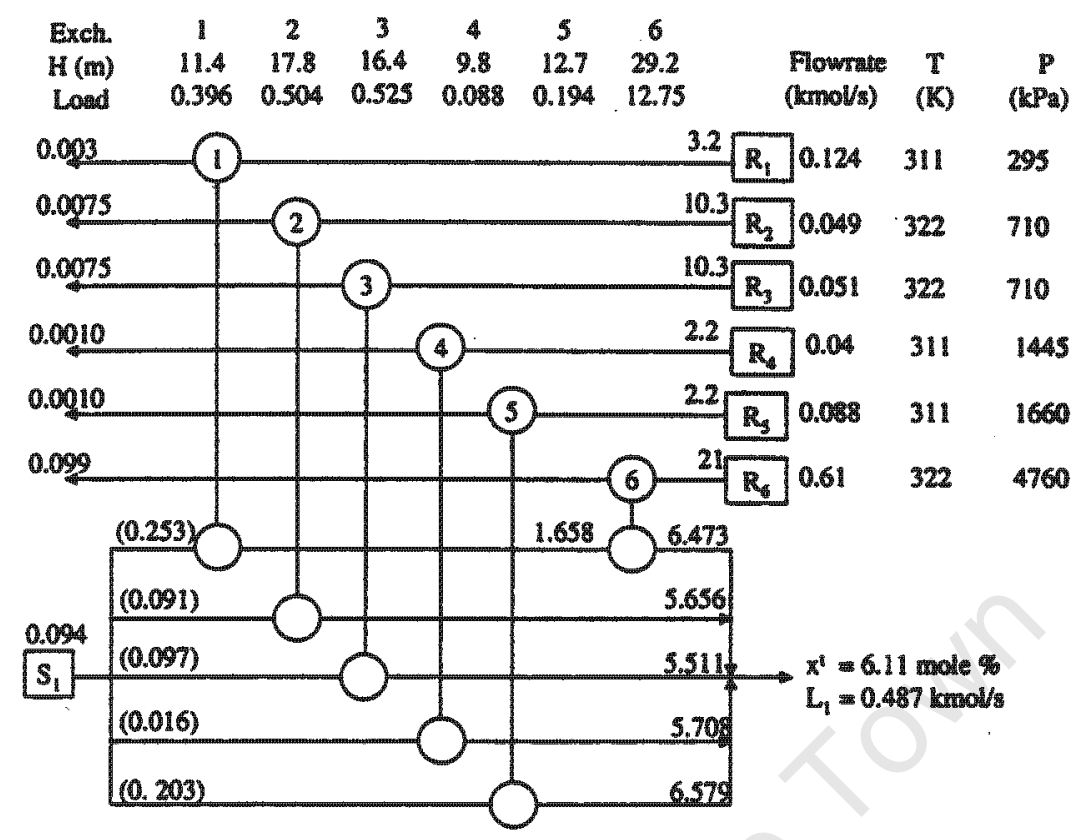

Figure 5.10: Totally integrated $\mathrm{H}_{2} \mathrm{~S}$ removal facility employing only MEA (N5)

has increased by $18 \mathrm{~m}$. The viability of connecting $S_{1}$ from mass exchanger 1 to mass exchanger 6 depends on the complexity of the plant layout and the distance between the two exchangers.

It is interesting to note the structure of a fully integrated flowsheet where the inlet composition of the DEA lean stream is allowed to be 0.04 mole\% as was discussed in the case of the $N 3$ option. Figure5.11 illustrates how the DEA is consumed in the matches with $R_{1}$, $R_{4}$ and $R_{5}$ and not with $R_{6}$ as in the original DEA flowsheet. The plant layout should tell if it is viable to connect $S_{2}$ from a DEA re-generator to mass exchanger 1,4 and 5 while the $\mathrm{H}_{2} \mathrm{~S}$ load to the MEA regenerator is increased due to treating $\mathrm{R}_{6}$ with the MEA.

The results for the different cases investigated are given in Table5.2. These results merely expose the many opportunities that may be considered to improve an operation at any stage in the life of the processing facility. These considerations are further enhanced by the availability of tools such as the hybrid models which can be used, interactively, to make decisions regarding the selection of potential alternatives. Figure5.6 gives an overview of the cost implications of these alternatives and from the onset it seems that the most attractive options to consider further are the DEA-replacement options - N1o+N4 and N5. These two options are the same and their implementation depends on the plant complexity and 


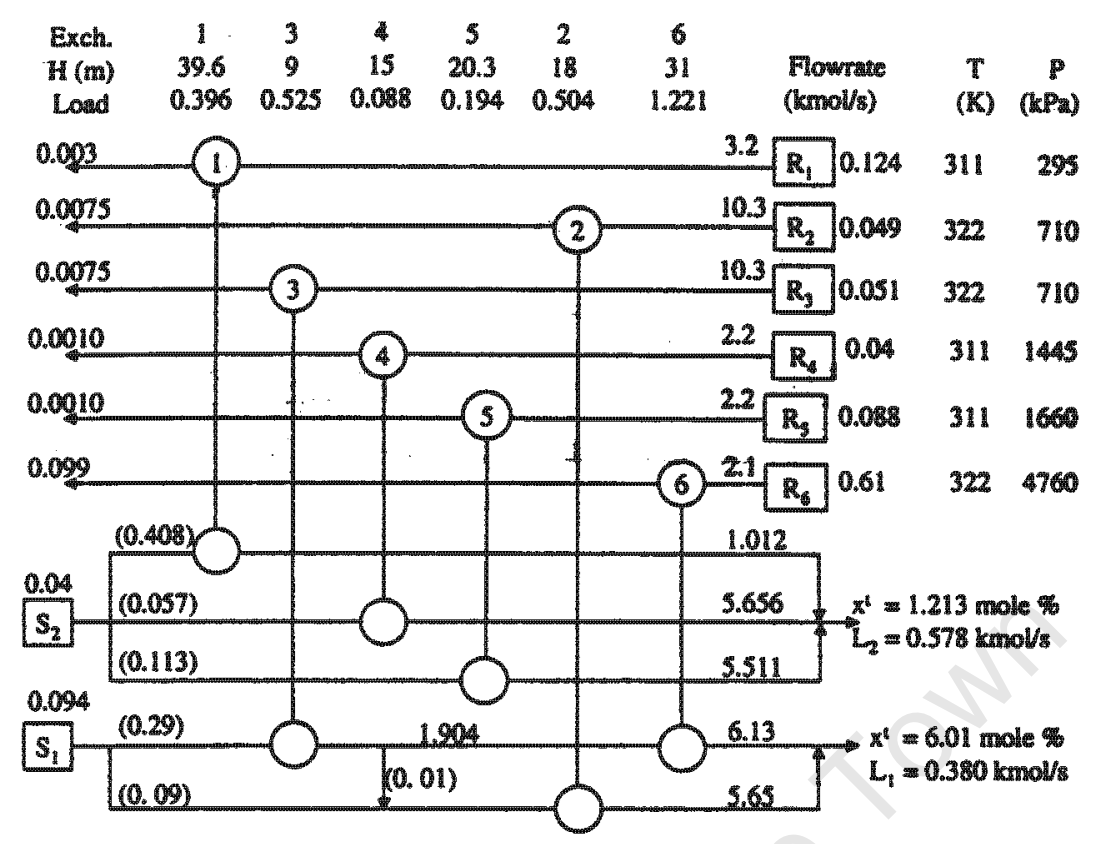

Figure 5.11: Totally integrated $\mathrm{H}_{2} \mathrm{~S}$ removal facility employing both MEA and DEA (N5 $+\mathrm{DEA})$

distance between the networks and the regeneration units. The MEA-regeneration unit may be closed down and the DEA-regeneration unit used instead. The DEA-regeneration unit has a liquid circulation rate of $29.8 \times 10^{3} \mathrm{~m}^{3} / \mathrm{hr}$ and the circulation rate resulting from the $N 5$ network is $35.4 \times 10^{3} \mathrm{~m}^{3} / \mathrm{hr}$.

A detailed analysis of the circulation rates (Figure5.12) of the MSA in each of the other exchangers analysed in $N 1$ to $N 5$ should expose the ease of implementing any of the designs discussed. From the visual analysis of the circulation rates certain qualitative judgements can be made regarding the feasibility of implementing a particular design option.

\subsection{Summary}

The hybrid model has been applied to an industrial problem where the improvements to the operating conditions are subject to constrained changes to the geometry of the processing facility. Four interconnection scenarios were investigated. The first one concerned the synthesis of the a process facility in which separate regions were not integrated. The second and third scenarios considered the option of using an alkanolamine from a neighbouring 


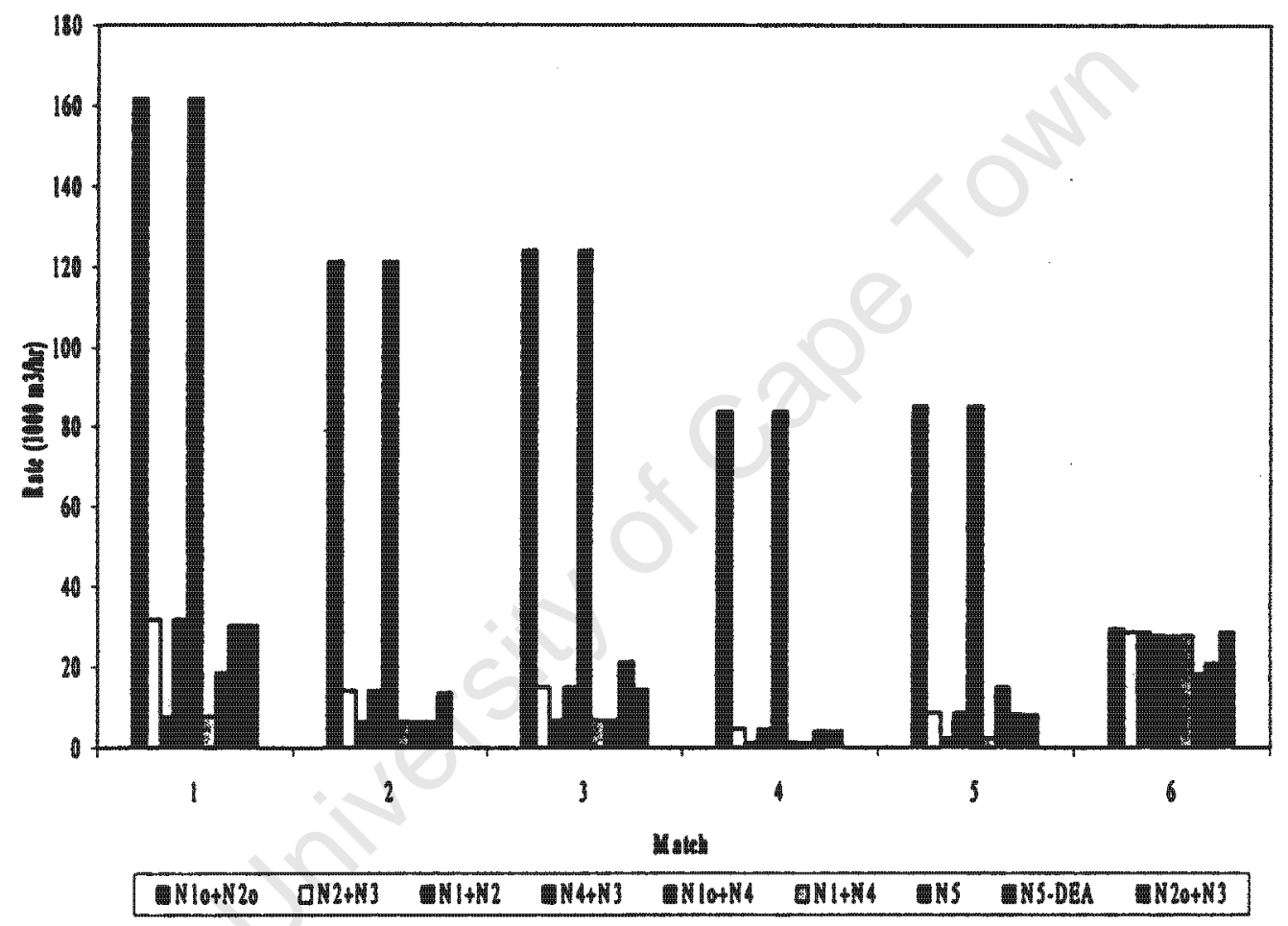

Figure 5.12: Analysis of the resulting circulation rates from various design options 
region as an alternative MSA in another region. Finally the chapter considered the option of generating a totally integrated processing facility while minimising the changes to the flowsheet.

The fully integrated network costed less than the separated flowsheet. A knowledge of the plant layout should reveal the benefits if any of implementing either the integrated system or the separated networks. These benefits will be subject to the cost of interconnection pipelines.

The tests in this chapter required the knowledge of the equilibrium relations of the reacting species. Vapour-liquid equilibrium data were generated using the Deshmuk-Mather prediction-model that take into account the fugacities and interaction parameters of the reacting species in the sweetening process. Making these models available to the process synthesis engineer allows for various alternatives to be studied without the need to undertake expensive and intensive experimental programmes. 


\section{Chapter 6}

\section{Conclusions}

Casca: Nay, an' I tell you that I'll ne'er look you i' the face again; but those. that understood him smiled at one another and shook their heads; but for mine own part, it was Greek to me.

William Shakespeare (1564-1616)

A hybrid tool for the synthesis of mass exchange networks has been presented. The tool uses pinch targets to improve solutions generated by a mixed-integer non-linear program model. Composition intervals are used to decide on the size of the mathematical model. The involvement of the engineer is the major part of the synthesis algorithm. Pinch-based driving force diagrams are used to help the designer visualise areas of improvement in an optimisation solution. The hybrid model has been shown to be able to reach pinchbased targets to within $10 \%$ and this is an improvement from the MINLP-based solutions previously reported.

The hybrid model exposed modelling issues that could hamper the successful achievement of optimal MINLP solutions. Methods of implementing logical operators for the determination of the number of trays of staged vessels were proposed and their merits analysed. Insights from the construction of a step in the McCabe-Thiele diagram have been used to model an analytical method that would approximate the number of stages in an exchanger when the vapour-liquid equilibrium functions are nonlinear.

An industrial problem was solved to illustrate use of the hybrid model in solving problems with constrained topology changes. Integrated in the data reconciliation step of this problem was the generation of vapour-liquid equilibrium data. The use of models to predict 
important thermodynamic and kinetic parameters obviates the need to carry out expensive experimental work while also allowing for many alternative processing routes to be examined in finite time.

The hybrid tool has exposed certain limitations that need to be alleviated as part of continued research. The method requires good initialisation strategies These initial solutions cannot be the optimal pinch-based solutions as it was used in this work because pinch solutions are already near-optimal and the use of computationally expensive tools to further improve the already near-optimal solutions cannot be justified.

The development of ad hoc logical operators hinders the process of solving many industrial problems. Thus there is a need for the development of modelling tools that can handle boolean and conditional functions. Efforts are currently been made at Carnegie Mellon where algorithms to solve Disjunctive Programming problems are being developed.

As with the many previously solved MINLP problems, the need for a robust global optimisation algorithm still remains. The pinch targets used in this thesis attempted to drive the MINLP solutions to optimality but the internal limitations of the MINLP solvers themselves meant that every problem would require the successful determination of targets. This highlights an area in pinch analysis that requires development: the graphical tools to aid the designer in analysing multiple tradeoffs. This limitation is evident in the regeneration and the retrofit examples when selecting the optimal minimum composition difference for both the regeneration and primary networks. It can be perceived that problems with many streams will be hindered by this lack of graphical tools.

Prediction packages for vapour-liquid equilibrium data must become an important part of process synthesis as more and more industrial problems (with limited, proprietary or simply unreliable data) are solved. The development of these models for common industrial processes can be perceived as an extension into process synthesis as product development gets included in the onion diagram. At a recent ESCAPE-11 conference(?) presented the notion of using thermodynamic models to predict important data and component properties as a being a cost saving exercise in the development of a process. In a greater context, computational chemistry and process design tools should be iteratively used to predict optimal process flowsheets. Evidence of such research directions is also notable from the ESCAPE-11 conference where Cummings(2001) briefly described the ways in which these tools are being used today and will be used in the future, to facilitate process and product design while Pantelides(2001) identified some key outstanding problems in multi- 
scale modelling. It is therefore perceivable that during this transition towards improving existing facilities, the objective function will in addition to cost, concern the quality of products and the selectivity of reacting species as well.

The generation of the equilibrium function exposed the effect of having incompatible components $\left(\mathrm{CO}_{2}\right.$ in the $\mathrm{H}_{2} \mathrm{~S}$ removal) during a mass exchange process. The synthesis of mass exchange networks when considering incompatible problems has not been fully investigated as yet. Previous approaches to this problem included the assumption of a key component that controls the mass transfer and all the other components would then be expressed in terms of this key component. Alternatively a pseuda-component can be assumed that will include all the incompatible components and then any or a combination of the synthesis approaches can be used to generate the mass exchange network. 


\section{References}

Alva-Argaez, A. (1999). Integrated design of water systems. $\mathrm{PhD}$ thesis, University of Manchester Institute of Science and Technology.

Bagajewicz, M. J., Pham, R., and Manousiouthakis, V. (1998). On the state space approach to mass/heat exchanger network design. Chem. Eng. Sci., 53(14):2595-2621.

Benko, N., Rev, E., and Fonyo, Z. (2000). The use of nonlinear programming to optimal water allocation. Chem. Eng. Comm., 178:67-101.

Brooke, A., Kendric, D., and Meeraus, A. (1988). GAMS, A user's guide. The Scientific Press, San Francisco.

Chen, J. (1987). Letter to the editor: Comments on improvement on a replacement for the logarithmic mean. Chem. Eng. Sci, 42(2488).

Comeaux, G. R. (2000). Synthesis of mass exchange networks with minimum total cost. Master's thesis, University of Machester Institute of Science and Technology.

Deshmuk, R. D. and Mather, A. (1981). A mathematical model for equilibrium solubility of hydrogen sulfide and carbon dioxide in aqueous alkanolamine solutions. Chem. Eng. Sci., 36:355-362.

El-Halwagi, M. (1989). Synthesis of mass exchange networks. AIChE J., 35(8):1233-1244.

El-Halwagi, M. and Srinivas, B. (1992). Synthesis of reactive mass-exchange networks. Chem. Eng. Sci., 47(8):2113-2119.

El-Halwagi, M. M. (1997). Pollution Prevention through Process Integration:Systematic Design Tools. Academic Press, San Diego. 
El-Halwagi, M. M. and Manousiouthakis, V. (1990a). Automatic synthesis of mass exchange networks with single-component tagets. Chem. Eng. Sci., 45(9):2813-2831.

El-Halwagi, M. M. and Manousiouthakis, V. (1990b). Simulteneous synthesis of mass exchange and regeneration networks. AIChe J, 36(8):1209-1219.

Floudas, C. A. (1995). Nonlinear and Mixed-Integer Optimization:Fundamentals and Apllications. Oxford University Press, New York.

Floudas, C. A. and Ciric, A. R. (1989). Strategies for overcomming uncertainties in heat exchanger network synthesis. Comp. Chem. Eng., 13(10):1133-1152.

GAMS (1999). GAMS-The Solvers Manuals. GAMS Development Corporation.

Grossmann, I. and Daichendt, M. M. (1996). New trends in optimisation-based approaches to process synthesis. Comp. Chem. Eng., 20(6-7):665-683.

Gupta, A. and Manousiouthakis, V. (1993). Minimum utility cost of mass exchange networks with variable sinle component supplies and targets. Ind. Eng. Chem. Res., 32(9):1937-1950.

Hallale, N. (1998). Capital cost targets for the optimum synthesis of mass exchange networks. $\mathrm{PhD}$ thesis, University of Cape Town.

J.D., L. and W., G. A. (1976). Gas sweetening data: Equilibrium solubility of Hydrogen Sulphide and Carbon Dioxide in aqueous Monoethanolamine and aqueous Diethanolamine solutions. J. Chem. Eng. Data, 21(1):20-30.

Jones, J. H. and Claytor, E. E. (1959). Solubility of acidic gases in aqueous Monoethanolamine. J. Chem. Eng. Data, 4:85-92.

Kent, R. L. and Eisenberg, B. (1976). Better data for amine treating. Hydrocarbon Processing, pages 87-90.

King, C. (1980). Separation Processes. McGraw-Hill, New York, 2d edition.

Kravanja, Z. and Glavic, P. (1997). Cost targeting for hen through simulteneous optimisation: a unified pinch technology and mathematical programming design of large hen. Comp. Chem. Eng., 21(8):833-853. 
Lee, J. I., Otto, F. D., and Mather, A. E. (1974). Solubility of h2s and co2 in aqueous Monoethanolamine solutions. Can. J. Chem. Eng., 52:803-805.

Lee, S. and Park, S. (1996). Synthesis of mass exchange network using process graph theory. Comp. Chem. Eng, 20(S):S201-S205.

Linnhoff, B. (1993). Pinch Analysis:Astate-of-the-art overview. Trans. IChemE, 71:503522.

Papalexandri, K. P., Pistikopoulos, E. N., and Floudas, C. A. (1994). Mass exchange networs for waste minimisation: a simulteneous approach. Trans. Inst. Chem. Eng, 72(Part A):279-294.

Smith, R. (1995). Chemical Process Design. McGraw-Hill, Singapore.

Srinivas, B. K. and El-Halwagi, M. M. (1994). Synthesis of reactive mass-exchange networks with general nonlinear equilibrium function. AIChe J., 40(3):463-472.

Treybal, R. E. (1981). Mass Transfer Operations. McGraw-Hill, Singapore, 3 edition.

Wang, Y. and Smith, R. (1994). Wastewater minimisation. Chem. Eng. Sci, 49:981-1006.

Wilson, S. and Manousiouthakis, V. (1998). Minimum utility cost for a multicomponent mass exchange operation. Chem. Eng. Sci, 53(22):3887-3896.

Zhu, X. X. (1995). Automated design method for heat exchanger networks using block decomposition and heuristic rules. Comp. Chem. Eng., 21:1095. 


\section{Appendix A}

\section{Stream Data}

$\mathrm{SO}_{2}$ removal

Table A.1: Stream data for $\mathrm{SO}_{2}$ removal: Base case

\begin{tabular}{|c|c|c|c|c|c|}
\hline Rich & $\begin{array}{c}G \\
(k m o l / h r)\end{array}$ & $\begin{array}{c}y^{3} \\
(\mathrm{kmol} / \mathrm{kmol} \text { inert })\end{array}$ & $\begin{array}{c}y^{t} \\
(k m o l / k m o l \text { inert })\end{array}$ & $\begin{array}{c}\text { Diameter } \\
m^{2}\end{array}$ & $\begin{array}{c}\text { Spacing } \\
m\end{array}$ \\
\hline $\mathrm{R}_{1}$ & 50 & 0.01 & 0.004 & 0.64 & 0.5 \\
\hline$R_{2}$ & 60 & 0.01 & 0.005 & 0.7 & 0.5 \\
\hline $\mathrm{R}_{3}$ & 40 & 0.02 & 0.0005 & 0.58 & 0.5 \\
\hline $\mathrm{R}_{4}$ & 30 & 0.02 & 0.015 & 0.5 & 0.5 \\
\hline MSAs & $\begin{array}{c}L^{c} \\
(\mathrm{kmol} / \mathrm{hr})\end{array}$ & $\begin{array}{c}x^{s} \\
(\mathrm{kmol} / \mathrm{kmol} \text { inert })\end{array}$ & $\begin{array}{c}x^{t} \\
(\mathrm{kmol} / \mathrm{kmol} \text { inert })\end{array}$ & $\mathrm{m}$ & $\mathrm{b}$ \\
\hline$S_{1}$ & $\infty$ & 0 & - & 26.1 & -0.00326 \\
\hline
\end{tabular}

Table A.2: Cost data for $\mathrm{SO} 2$ removal: Base case Column capital cost

Shell cost (installed) $\$ 12800 H_{t}^{0.95} D^{0.6}$

$$
\begin{array}{rl}
\text { Trays } & \$ 608 \mathrm{e}^{0.8 D} \text { per tray } \\
& D=\text { column diameter }(\mathrm{m}) \\
& H_{t}=\text { total column height }(\mathrm{m}) \\
E_{0} & 20 \% \\
\text { Inactive height } & 3 \mathrm{~m}
\end{array}
$$


Table A.3: Stream data for $\mathrm{SO}_{2}$ removal: Scenario2

\begin{tabular}{|cccccc}
\hline Rich & $\begin{array}{c}G \\
(\mathrm{kmol} / \mathrm{hr})\end{array}$ & $\begin{array}{c}y^{s} \\
(\mathrm{kmol} / \mathrm{kmol} \text { inert })\end{array}$ & $\begin{array}{c}y^{t} \\
(\mathrm{kmol} / \mathrm{kmol} \text { inert })\end{array}$ & $\begin{array}{c}\text { Diameter } \\
\mathrm{m}^{2}\end{array}$ & $\begin{array}{c}\text { Spacing } \\
\mathrm{m}\end{array}$ \\
\hline $\mathrm{R}_{1}$ & 50 & 0.01 & 0.004 & 0.66 & 0.342 \\
$\mathrm{R}_{2}$ & 60 & 0.01 & 0.005 & 0.72 & 0.407 \\
$\mathrm{R}_{3}$ & 40 & 0.02 & 0.0005 & 0.59 & 0.273 \\
$\mathrm{R}_{4}$ & 30 & 0.02 & 0.015 & 0.51 & 0.204 \\
& & & & & \\
\hline MSAs & $L^{c}$ & $x^{s}$ & $x^{t}$ & $\mathrm{~m}$ & $\mathrm{~b}$ \\
& $(\mathrm{kmol} / \mathrm{hr})$ & $(\mathrm{kmol} / \mathrm{kmol}$ inert $)$ & $(\mathrm{kmol} / \mathrm{kmol}$ inert $)$ & & \\
\hline $\mathrm{S}_{1}$ & $\infty$ & 0.0002 & - & 26.1 & -0.00326 \\
& & & & & \\
\hline
\end{tabular}

Table A.4: Equipment data for $\mathrm{SO}_{2}$ removal: Scenario2 Column capital cost

Shell cost (installed) $\$ 12800 H_{t}^{0.95} D^{0.6}$

Trays $\$ 608 \mathrm{e}^{0.8 D}$ per tray $D=$ column diameter $(m)$ $H_{t}=$ total column height $(\mathrm{m})$

$K_{y} a \quad 0.02 \mathrm{kmol} \mathrm{SO}_{2} / \mathrm{m}^{3} / \mathrm{s}$

Inactive height $3 \mathrm{~m}$

Table A.5: Stream data for dephenolisation: base case

\begin{tabular}{cccccccc}
\hline $\begin{array}{c}\text { Rich } \\
\text { streams }\end{array}$ & $\begin{array}{c}G \\
(\mathrm{~kg} / \mathrm{s})\end{array}$ & $\begin{array}{c}y^{s} \\
\text { mass frac. }\end{array}$ & $\begin{array}{c}y^{t} \\
\text { mass frac. }\end{array}$ & $\begin{array}{c}\text { Density } \\
\left(\mathrm{kg} / \mathrm{m}^{3}\right)\end{array}$ & & & \\
\hline $\mathrm{R}_{1}$ & 2 & 0.050 & 0.10 & 1000 & & & \\
$\mathrm{R}_{2}$ & 1 & 0.03 & 0.006 & 1000 & & & \\
\hline & & & & & & & \\
\hline MSAs & $L^{c}$ & $x^{s}$ & $x^{t}$ & $\begin{array}{c}\text { Density } \\
\left(\mathrm{kg} / \mathrm{m}^{3}\right)\end{array}$ & & $\mathrm{m}$ & $\begin{array}{c}\text { Cost } \\
(\mathrm{k} / \mathrm{yr}) /(\mathrm{kg} / \mathrm{s})\end{array}$ \\
\hline $\mathrm{S}_{1}$ & 5 & 0.005 & 0.015 & 880 & 2 & 0 & 0 \\
$\mathrm{~S}_{2}$ & 3 & 0.01 & 0.030 & 930 & 1.53 & 0 & 0 \\
$\mathrm{~S}_{3}$ & $\infty$ & 0.0013 & 0.015 & 830 & 0.71 & 0.001 & 293.4 \\
\hline
\end{tabular}


Table A.6: Equipment data for dephenolisation: base case

Exchanger cost (installed) $\$ 9050 V^{0.6}$ (volume in $\mathrm{m}^{3}$ )

T 10 minutes per stage

$E_{0} \quad 100 \%$

Annualisation factor 0.2

Table A.7: Stream data for dephenolisation: mixed-equipment

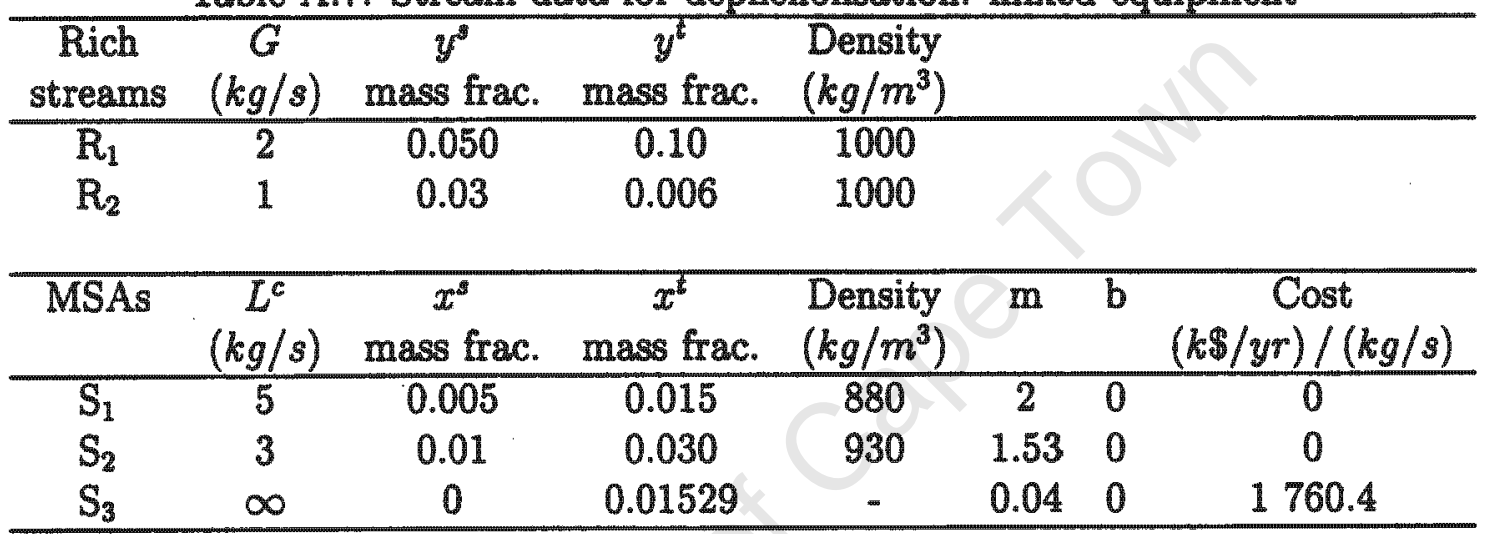

Table A.8: Equipment data for dephenolisation: mixed-equipment

Mixed settlers:

$\tau: \quad 10$ minutes per stage

$100 \%$

Installed cost (carbon steel): $\$ 9050 V^{0.6}\left(V\right.$ in $\mathrm{m}^{3}$ )

Installed cost (stainless steel): $\$ 18100 V^{0.6}\left(V\right.$ in $\left.\mathrm{m}^{3}\right)$

Tray columns:

$K_{W g}: \quad 0.0057 \mathrm{~kg} / \mathrm{s} / \mathrm{kg}$ exchanger for carbon steel $0.007 \mathrm{~kg} / \mathrm{s} / \mathrm{kg}$ exchanger for stainless steel

Shell cost (carbon steel, installed): $\$ 618 M^{0.66}(\mathrm{M}$ in $\mathrm{kg}$ )

Shell cost (stainless steel, installed): $\$ 1236 M^{0.66}(\mathrm{M}$ in $\mathrm{kg}$ )

Tray costs: $20 \%$ of shell

Annualisation factor: 0.2 (all equipment) 
Table A.9: Stream data for regeneration example

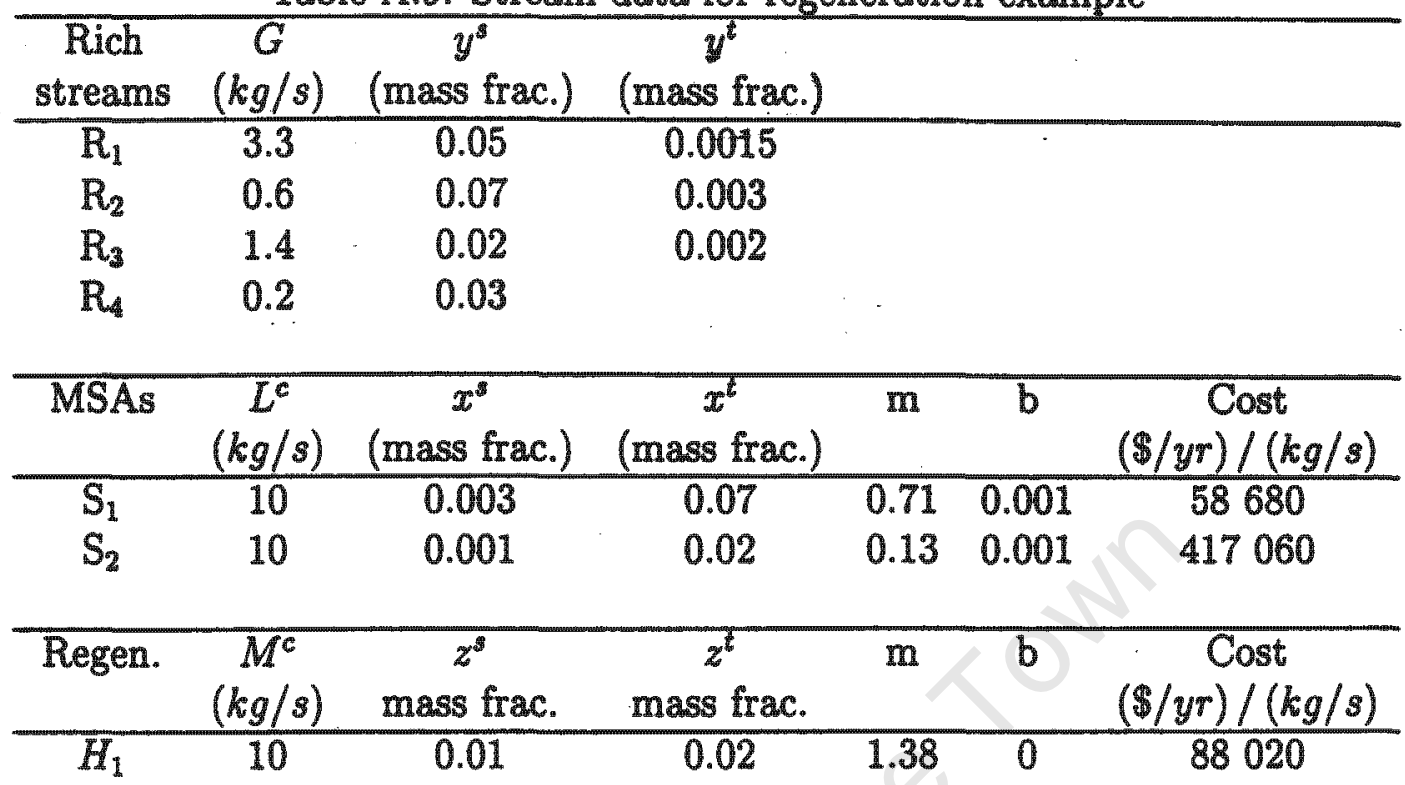

Table A.10: Equipment data for regeneration case

Tray column cost(installed): $\$ 4552 N_{\text {stages }} / y r$

Packed columns:

Diameter: $1 \mathrm{~m}$

Installed cost: $\$ 4245 \mathrm{H} / \mathrm{yr}$

$H=$ packed height (m)

Table A.11: Stream data for REAMEN example with DEA

\begin{tabular}{ccccc}
\hline $\begin{array}{c}\text { Rich } \\
\text { streams }\end{array}$ & $\begin{array}{c}G \\
\left(\mathrm{~m}^{3} / \mathrm{s}\right)\end{array}$ & $\begin{array}{c}y^{s} \\
\left(\mathrm{kmol} / \mathrm{m}^{3}\right)\end{array}$ & $\begin{array}{c}y^{t} \\
\left(\mathrm{kmol} / \mathrm{m}^{3}\right)\end{array}$ \\
\hline $\mathrm{R}_{1}$ & 0.87 & $1.3 \mathrm{E}-5$ & $2.2 \mathrm{E}-7$ \\
$\mathrm{R}_{2}$ & 0.1 & $9 \mathrm{E}-6$ & $2.2 \mathrm{E}-7$ & \\
& & & & $x^{3}$ \\
\hline MSAs & $L^{\mathrm{c}}$ & $x^{s}$ & $x^{t}$ & Cost \\
& $\left(\mathrm{m}^{3} / \mathrm{s}\right)$ & $\left(\mathrm{kmol} / \mathrm{m}^{3}\right)$ & $\left(\mathrm{kmol} / \mathrm{m}^{3}\right)$ & $(\$ / y r) /\left(\mathrm{m}^{3} / \mathrm{s}\right)$ \\
\hline $\mathrm{S}_{1}$ & 0.0002 & 0 & 0.015 & 0 \\
$\mathrm{~S}_{2}$ & $\infty$ & $2 \mathrm{E}-6$ & $2 \mathrm{E}-3$ & $8.80833 \mathrm{E} 8$ \\
$\mathrm{~S}_{3}$ & $\infty$ & $1 \mathrm{E}-6$ & $2 \mathrm{E}-6$ & $9.94039 \mathrm{E} 8$ \\
\hline
\end{tabular}


Table A.12: Stream data for RAEMEN example with MEA

\begin{tabular}{ccccc}
\hline $\begin{array}{c}\text { Rich } \\
\text { streams }\end{array}$ & $\begin{array}{c}G \\
(\mathrm{~kg} / \mathrm{s})\end{array}$ & $\begin{array}{c}y^{g} \\
\text { (mass frac.) }\end{array}$ & $\begin{array}{c}y^{t} \\
\text { (mass frac.) }\end{array}$ & $\begin{array}{c}K_{W g} \\
(\mathrm{~kg} / \mathrm{s} / \mathrm{kg} \text { exchanger) }\end{array}$ \\
\hline$R_{1}$ & 10 & 0.03 & 0.005 & 0.008 \\
$R_{2}$ & 10 & 0.01 & 0.005 & 0.005 \\
& & & & \\
\hline MSAs & $L^{c}$ & $x^{s}$ & $x^{t}$ & \\
& $(\mathrm{~kg} / \mathrm{s})$ & (mass frac.) & (mass frac.) \\
\hline$S_{1}$ & $\infty$ & 0.01113 & 0.0474 & \\
\hline
\end{tabular}

Table A.13: Equipment data for REAMEN example with MEA Shell cost (installed) $\$ 618 M^{0.66}(\mathrm{M}$ in $\mathrm{kg}$ )

Tray cost: $20 \%$ of shell 


\section{Appendix B}

\section{Basic sizing expressions}

There are two common types of technologies that are used in MENS: continuous and staged columns. In continuous contact exchangers, the rich and lean phases are in continuous contact with no intermediate phase separation or re-contacting. These exchangers are sized in terms of height. Staged mass exchangers consist of a series of stages in which a rich phase and a lean phase is repeatedly contacted and separated, usually in a countercurrent manner. With thorough mixing and sufficient time, the two phases leaving the stage will be in equilibrium with one another. The sizing of this type of mass exchange units therefore depends on the number of stages. The important sizing models which can be used in the objective function are briefly described in this section. The reader can also refer to the work of Hallale (1998) for additional details of the models bearing in mind that the models in this section differ only in application from those of Hallale. In Hallale the models are based on exchangers in a composition interval while in this section they are adapted for implementation in the optimisation environment. All the equations refer to sizing an exchanger $i j s$ with known geometrical properties and properties for the material of construction. The indices refer to the rich stream $i$ matching for the $s^{\text {th }}$ time with a lean stream $j$. The operating and equilibrium relations are linear except where reference is made to the method of approximating McCabe-Thiele construction for the determination of stages numbers $N s t_{i j s}$.

\section{Exchanger Height}

$$
H_{i j s}=H T U_{i j s} N T U_{i j s}
$$




$$
\begin{gathered}
\qquad N U_{i j s}=\frac{y_{i j s}^{I}-y_{i j s}^{O}}{\Delta y_{l m, i j s}} \\
\text { where } \Delta y_{l m, i j s}=\left(0.5\left(\Delta y_{i j s}^{I}+\Delta y_{i j s}^{O}\right)\left(\Delta y_{i j s}^{I}\right)\left(\Delta y_{i j s}^{O}\right)\right)^{0.333} \\
H T U_{i j s}=\frac{M_{e, i j s}}{S_{i} K_{y} a_{i}}
\end{gathered}
$$

EquationB.0.2 (Chen, 1987) is necessary in optimisation to prevent divisions by zero from the normal expression of the logarithmic mean. The column cross sectional area $S_{\mathrm{i}}$ and mass transfer coefficient are declared in terms of the rich streams. Where these parameters are constant through out the process, the index $i$ is dropped.

\section{Number of stages}

$$
\begin{aligned}
& N s t_{i j s}=\quad \frac{\ln Y r_{i j s}}{\ln A_{i j s}} \bigcirc \begin{array}{l}
A_{i j s} \neq 1 \\
N s t_{i j s}=\frac{y_{i j s}^{I}-y_{i j s}^{o}}{y_{i j s}^{O}-m_{i j} x_{i j s}-b_{i j}}
\end{array} \\
& A_{i j s}=1 \\
& \text { where } Y r_{i j s}=\frac{y_{i j s}^{I}-m_{i j} x_{i j s}^{O}-b_{i j}}{y_{i j s}^{O}-m_{i j} x_{i j s}^{I}-b_{i j}}
\end{aligned}
$$

Mass of continuous contactor $M_{p s m, i j s}$

$$
\begin{gathered}
M_{p s m, i j s}=\frac{M_{e, i j s}}{K_{w} \Delta y_{l m, i j s}} \\
\text { where } K_{w}=\frac{K_{y} a\left(2 J f-P_{i}\right)}{4 P_{i} p_{m}\left(1+f_{i}\right)\left(1+f_{e}\right)\left(1+f_{c}\right)}
\end{gathered}
$$

The parameters that are lumped into the coefficient $K_{w}$ are assumed to be constant and only a function of the material of construction of the mass exchangers

$K_{y} a \quad$ the mass transfer coefficient 
$J \quad$ the welded factor

$f$ the design stress for construction material at the design temperature

$P_{i} \quad$ the internal design pressure

$\rho_{m} \quad$ density of the design material

$f_{i} \quad$ the fractional allowance for inactive height

$f_{e} \quad$ the fractional allowance for extras such as manholes, skirts,nozzles etc. and

$f_{c}$ the fractional allowance for corrosion

Mass of stagewise contactor $M_{p s m, i j s}$

$$
M_{t s m, i j s}=\frac{g_{i j s}^{E} N s t_{i j s}}{K_{w}}
$$

The mass transfer coefficient $K_{w}$ and the flowrate of rich stream $i, g_{i j s}^{E}$ are replaced by $K_{w g}$ or $K_{w l}$ and $l_{i j s}^{E}$ depending on the phases of the contacting streams. The mass transfer coefficients, $K w g$ and $K w l$ are given by EquationsB.0.8andB.0.9.

$$
\begin{aligned}
& K_{w g}=\left(\frac{E_{o} \rho_{v} u_{v}}{s}\right) \frac{\left(2 J f-P_{i}\right)}{4 P_{i} \rho_{m}\left(1+f_{i}\right)\left(1+f_{e}\right)\left(1+f_{c}\right)} \\
& K_{w b}=\left(\frac{d_{o}^{2} h E_{o} \rho_{d} v_{o}}{26.7 p^{2} s}\right) \frac{\left(2 J f-P_{i}\right)}{P_{i} \rho_{m}\left(1+f_{i}\right)\left(1+f_{e}\right)\left(1+f_{c}\right)}
\end{aligned}
$$

where:

$E_{o}$ is the overall efficiency

$\rho$ is the gas stream or dispersed phase density

$s$ is the tray spacing given by $0.5 D^{0.3}$ for exchangers with $D>1 \mathrm{~m}$ and $0.5 \mathrm{~m}$ for smaller diameters

$u_{v}$ is the superficial velocity whilst 
$v_{0}$ is the velocity of the dispersed phase through a hole

$d_{0}$ is the diameter of the holes in the tray

$p$ is the hole pitch and

$h$ is a shape factor for holes on the pitch-3.62 for a triangular pinch and 3.14 for a square pitch

\section{Exchanger Volume $V_{i j s}$}

For mixed settlers, the sizing model is a function of exchanger volume according to EquationB.0.10:

$$
V_{i j s}=\frac{\tau N_{s t, i j s}}{E_{o}}\left(\frac{g_{i j s}^{E}}{\rho_{i}}+\frac{l_{i j s}^{E}}{\rho_{j}}\right)
$$

where

$\tau$ is the residence time per stage

$\rho$ is the density for the rich, $i$ and the lean, $j$ streams

The hybrid model implements EquationsB.0.6toB.0.10 for the sizing of mass exchangers. 
Number of stages for non-linear equilibrium functions

The following non-linear forms of the equilibrium functions can be fitted used according EquationsB.0.13 to B.0.14 approximate the number of stages determined from a McCabeThiele construction.

$$
\begin{aligned}
& y=a_{1} x^{b_{1}}+a_{2} x^{b_{3}}+\ldots \\
& y=a_{1} e^{b_{1} x}
\end{aligned}
$$

$$
\begin{gathered}
N s t_{i j s}=\frac{\left(y_{i j s}^{I}-y_{i j s}^{O}\right)}{\frac{1}{2} O m_{i j s}\left(x_{i j s}^{O}+x_{i j s}^{I}\right)+o b_{i j s}}-\frac{\left(y_{i j s}^{I}-y_{i j s}^{O}\right)\left(x_{i j s}^{O}-x_{i j s}^{I}\right)}{\frac{a}{b_{1}+1}\left[\left(x_{i j s}^{O}\right)^{b_{1}+1}-\left(x_{i j s}^{I}\right)^{b_{1}+1}\right]}-\ldots \\
N s t_{i j s}=\frac{\left(y_{i j s}^{I}-y_{i j s}^{O}\right)}{\frac{1}{2} O m_{i j s}\left(x_{i j s}^{O}+x_{i j s}^{I}\right)+o b_{i j s}}-\frac{\left(y_{i j s}^{I}-y_{i j s}^{O}\right)\left(x_{i j s}^{O}-x_{i j s}^{I}\right)}{\frac{a_{1}}{b_{1}}\left[e^{b_{1} x_{i j s}^{O}}-e^{\left.b_{1} x_{i j s}^{I}\right]}\right.}
\end{gathered}
$$




\section{Appendix C}

\section{MINLP Model Equations}

The actual equations used by Papalexandri et al. (1994) are repeated here to stream line the developments in this thesis. The model consist of:

Overall mass balances at the initial splitter of each stream

$$
\begin{array}{ll}
\sum_{\left(j \in L M_{i}\right), s} g_{i j s}^{I}-G_{i}=0 & i \in R \\
\sum_{\left(i \in R M_{j}\right), s} l_{i j s}^{I}-L_{j}=0 & j \in L
\end{array}
$$

Overall mass balances at the mixer preceding each mass exchanger for each stream

$$
\begin{array}{cc}
g_{i j s}^{I}+\sum_{\left(j^{\prime} \in R Y_{i j}\right), s^{\prime}} g_{j j^{\prime} s s^{\prime}}^{B i}-g_{i j s}^{E}=0 & (i, j) \in M \\
l_{i j s}^{I}+\sum_{\left(i^{\prime} \in L Y_{i j}\right), s^{\prime}} l_{i i^{\prime} s s^{\prime}}^{B j}-l_{i j s}^{E}=0 & (i, j) \in M
\end{array}
$$

Overall mass balances at the splitter after each exchanger for each stream

$$
\begin{array}{ll}
g_{i j s}^{O}+\sum_{\left(j^{\prime} \in R Y_{i j^{\prime}}\right), s^{\prime}} g_{j^{\prime} j j^{\prime} s}^{B i}-g_{i j s}^{E}=0 & (i, j) \in M \\
l_{i j s}^{O}+\sum_{\left(i^{\prime} \in L Y_{i^{\prime} j}\right), s^{\prime}} l_{i^{\prime} i s^{\prime} s}^{B j}-l_{i j s}^{E}=0 & (i, j) \in M
\end{array}
$$


Mass balances for the transferable component at the mixer preceding each exchanger for each stream

$$
\begin{array}{ll}
g_{i j s}^{I} y_{i}^{s}+\sum_{\left(j^{\prime} \in R Y_{i j}\right), s^{\prime}} g_{j j^{\prime} s s^{\prime}}^{B i} y_{i^{\prime} s^{\prime}}^{O}-g_{i j s}^{E} y_{i j s}^{I}=0 & (i, j) \in M \\
l_{i j s}^{I} x_{j}^{s}+\sum_{\left(i^{\prime} \in L Y_{i j}\right), s^{\prime}} l_{i i^{\prime} s s^{\prime}}^{B j} x_{i^{\prime} j s^{\prime}}^{O}-l_{i j s}^{E} x_{i j s}^{I}=0 & (i, j) \in M
\end{array}
$$

Mass balances for the transferable component at the final mixer of each stream

$$
\begin{aligned}
& \sum_{\left(j \in L M_{i}\right), s} g_{i j s}^{O} y_{i j s}^{O}-G_{i} y_{i}^{T}=0 \quad i \in R \\
& \sum_{\left(i \in R M_{j}\right), s} l_{i j s}^{O} x_{i j s}^{O}-L_{j} x_{j}^{T}=0 \quad j \in L
\end{aligned}
$$

Mass balances for the transferable component at each potential exchanger for each stream

$$
\begin{array}{ll}
g_{i j s}^{E}\left(y_{i j s}^{I}-y_{i j s}^{O}\right)-M_{i j s}^{E}=0 & (i, j) \in M \\
l_{i j s}^{E}\left(x_{i j s}^{O}-x_{i j s}^{I}\right)-M_{i j s}^{E}=0 & (i, j) \in M
\end{array}
$$

\section{Feasibility constraints}

A minimum composition approach, $\varepsilon_{i, j}$ is specified to ensure feasible mass transfer.

$$
\begin{array}{ll}
m_{i j}\left(x_{i j s}^{O}+\varepsilon_{i j}\right)+b_{i j}-y_{i j s}^{I} \leq 0 & (i, j) \in M \\
m_{i j}\left(x_{i j s}^{I}+\varepsilon_{i j}\right)+b_{i j}-y_{i j_{s}}^{O} \leq 0 & (i, j) \in M
\end{array}
$$

The minimum composition approach should be set at a low enough value so that during optimisation the solution cannot be trapped at the lower bound of $\varepsilon_{i, j}$. In thesis an evaluation of the driving force for each match in the final network is carried out . 
Intuitively it would appear that in an optimisation problem there is no need to set a minimum value for $\varepsilon_{i, j}$ as too small a value will lead to networks that require a large capital investment. However, most solution search algorithms depend of the existence of smooth functions in the model. The Kremser equation or the number of transfer units model expressed in terms of the composition difference. Not placing a lower bound on this composition difference and allowing the solution search to propagate to regions where it evaluates the equipment size for exchangers that have a zero $\varepsilon_{\mathfrak{i}, j}$ values, the

$$
\ln \left(\frac{y_{i j s}^{I}-m_{i j} x_{i j s}^{O}-b_{i j}}{y_{i j s}^{O}-m_{i j} x_{i j s}^{I}-b_{i j}}\right)
$$

term in the Kremser expression (Equation2.9) will be indeterminate or the driving force based $\Delta y_{l m}$ in

$$
\Delta y_{l m}=\left(\left(\Delta y_{\text {in }} \Delta y_{\text {out }}\right)\left(\Delta y_{\text {in }}+\Delta y_{\text {out }}\right) / 0.5\right)^{\frac{1}{3}}
$$

will be zero and make the sizing of the exchanger impossible.

\section{Logical and connectivity constraints}

The logical constraint (EquationC.0.15) uses a big-M formulation, big- $U$ in this case, to ensure that only the continuous variables corresponding to exchangers that exist on the flowsheet are active at the final solution. The value of $U$ is merely specified as a large positive number. Recall that from Section2.3.2 this value is the minimum upper bound of available transferable mass between a lean and rich stream via Equation2.19.

$$
M_{i j s}^{E}-y_{i j s} U \leq 0 \quad(i, j) \in M
$$

EquationsC. 0.16 to C. 0.23 refer to the connectivity constraints. They are used for the selection of mixers and splitters in the final network.

$$
\begin{array}{ll}
g_{i j s}^{E}-y_{i j s} U \leq 0 & (i, j) \in M \\
g_{i j s}^{I}-y_{i j s} U \leq 0 & (i, j) \in M \\
g_{i j s}^{O}-y_{i j s} U \leq 0 & (i, j) \in M
\end{array}
$$




$$
\begin{gathered}
\sum_{\left(j^{\prime} \in R Y_{i j}\right) s^{\prime}} g_{j j^{\prime} s s^{\prime}}^{B i}-y_{i j s} U \leq 0 \quad(i, j) \in M \\
l_{i j s}^{E}-y_{i j s} U \leq 0 \quad(i, j) \in M \\
l_{i j s}^{I}-y_{i j s} U \leq 0 \quad(i, j) \in M \\
l_{i j s}^{O}-y_{i j s} U \leq 0 \quad(i, j) \in M \\
\sum_{\left(i^{\prime} \in L Y_{i j}\right) s_{s^{\prime}}} l_{i i^{\prime} s s^{\prime}}^{B j}-y_{i j s} U \leq 0 \quad(i, j) \in M
\end{gathered}
$$

Sizing equations for the mass transfer units

Various mass exchangers can be sized according to any of the models models described in SectionB.

\section{Bounds on the variables}

Bounded variables are the mass flowrate of each lean stream, its final composition and the final composition of the lean stream. The outlet composition from each exchanger are not bounded.

$$
\begin{aligned}
& L_{j} \leq L j^{U} \quad j \in L \\
& x_{j}^{T} \leq x_{j}^{U} \quad j \in L \\
& y_{i}^{T} \leq y_{i}^{U} \quad i \in R
\end{aligned}
$$

To ensure that the solution is found within finite time, for variables where for practical reasons they are known to be less that a certain amount, then this knowledge should be 
used to bound such variables Also, the bounding strategy becomes necessary to prevent the determination of unrealistic equipment sizes.

\section{Objective function}

The objective function of the MINLP model is to minimise the total annualised cost and determine simultaneously the optimal network configuration, MSA consumption and equipment sizes and is of the form:

$$
\min T A C=\sum_{j \in L}\left(A C_{j}\right)\left(L_{j}\right)+\sum_{(i, j) \in M p, s}\left(A C_{s t}\right)\left(N_{s t, i j s}\right)+\sum_{(i, j) \in M t, s}\left(A C_{h}\right)\left(H_{s t, i j s}\right)
$$




\section{Appendix D}

\section{Case Study Additional Information}

Table D.1: Stream data case study

\begin{tabular}{|c|c|c|c|c|c|c|c|}
\hline $\begin{array}{l}\text { Rich } \\
\text { Stream }\end{array}$ & $\begin{array}{c}G \\
(\mathrm{kmol} / \mathrm{s})\end{array}$ & $\begin{array}{c}y^{3} \\
(\text { mole \%) }\end{array}$ & $\begin{array}{c}y^{k} \\
\text { (mole \%) }\end{array}$ & $\begin{array}{l}{ }^{\mathrm{T}} \mathrm{C} \\
{ }^{\circ} \mathrm{C}\end{array}$ & $\underset{k P a}{\mathrm{P}}$ & $\begin{array}{l}\mathrm{D} \\
m\end{array}$ & 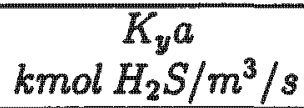 \\
\hline $\mathrm{R}_{1}$ & 0.124 & 3.2 & 0.003 & 38 & 295 & 1.0973 & 0.29 \\
\hline $\mathrm{R}_{2}$ & 0.049 & 10.3 & 0.0075 & 49 & 710 & 0.7925 & 0.16 \\
\hline $\mathrm{R}_{3}$ & 0.051 & 10.3 & 0.0075 & 49 & 710 & 0.987 & 0.11 \\
\hline$R_{4}$ & 0.04 & 2.2 & 0.001 & 38 & 1445 & 0.8405 & 0.21 \\
\hline $\mathrm{R}_{5}$ & 0.088 & 2.2 & 0.001 & 38 & 1760 & 1.067 & 0.22 \\
\hline$R_{6}$ & 0.61 & 21 & 0.099 & 49 & 700 & 1.2192 & 0.46 \\
\hline MSAs & $\begin{array}{c}L^{c} \\
(\mathrm{kmol} / \mathrm{s})\end{array}$ & $\begin{array}{c}x^{s} \\
\text { (mole \%) }\end{array}$ & $\begin{array}{c}x^{t} \\
\text { (mole \%) }\end{array}$ & & & & \\
\hline$S_{1}$ & 5 & 0.09373 & 10.3 & & & & \\
\hline $\mathrm{S}_{2}$ & 10 & 0.04 & 10.3 & & & & \\
\hline
\end{tabular}

*The maximum $\mathrm{H}_{2} \mathrm{~S}$ content is set by the problem specification. For DEA $\left(S_{2}\right)$ the maximum $\mathrm{H}_{2} S$ content is assumed to be similar to that of the MEA streams $\left(R_{1}\right.$ to $\left.R_{5}\right)$ 
Table D.2: Equipment data for gas sweetening case study Column capital cost Shell cost (installed) $\$ 618 M^{0.68}$

Tray Cost $20 \%$ of shell cost

Carbon steel:

$$
\text { Density } 7833 \mathrm{~kg} \cdot \mathrm{m}^{-3}
$$

Design stress $380 \mathrm{Pi}$

Inactive Height \% 0.15

Extras \% 0.2

Corrosion \% 0.5

Joint (J) \% 0.8

Table D.3: Partial Pressure and Loading data for the H2S-MEA system at $38^{\circ} \mathrm{C}$.

Match RIS1, RASI nd RSS1 Q T $=38$

Composwone are in mole\%

\begin{tabular}{|l|l|l|l|l|l|}
\hline PP & alpha & $x$ H2S & $y-R 4$ & $y-R 5$ & $y-R 1$ \\
\hline $5.00 E-06$ & $1.58 E-04$ & $1.09 E-03$ & $3.46 E-07$ & $3.01 E-07$ & $1.69 E-06$ \\
$8.00 E-06$ & $1.58 E-04$ & $1.09 E-03$ & $5.54 E-07$ & $4.82 E-07$ & $2.71 E-06$ \\
$8.85 E-06$ & $1.75 E-04$ & $1.20 E-03$ & $6.12 E-07$ & $5.33 E-07$ & $3.00 E-06$ \\
$8.85 E-05$ & $1.42 E-03$ & $9.77 E-03$ & $6.12 E-06$ & $5.33 E-06$ & $3.00 E-05$ \\
$8.85 E-04$ & $7.09 E-03$ & $4.86 E-02$ & $6.12 E-05$ & $5.33 E-05$ & $3.00 E-04$ \\
$1.45 E-03$ & $9.47 E-03$ & $6.50 E-02$ & $1.00 E-04$ & $8.70 E-05$ & $4.90 E-04$ \\
$1.66 E-03$ & $1.03 E-02$ & $7.04 E-02$ & $1.15 E-04$ & $1.00 E-04$ & $5.63 E-04$ \\
$8.85 E-03$ & $2.62 E-02$ & $1.79 E-01$ & $6.12 E-04$ & $5.33 E-04$ & $3.00 E-03$ \\
$1.45 E-02$ & $3.43 E-02$ & $2.35 E-01$ & $1.00 E-03$ & $8.70 E-04$ & $4.90 E-03$ \\
$1.66 E-02$ & $3.70 E-02$ & $2.53 E-01$ & $1.15 E-03$ & $1.00 E-03$ & $5.63 E-03$ \\
$8.85 E-02$ & $9.05 E-02$ & $6.18 E-01$ & $6.12 E-03$ & $5.33 E-03$ & $3.00 E-02$ \\
$8.85 E-01$ & $2.76 E-01$ & $1.86 E+00$ & $6.12 E-02$ & $5.33 E-02$ & $3.00 E-01$ \\
$8.85 E+00$ & $6.22 E-01$ & $4.09 E+00$ & $6.12 E-01$ & $5.33 E-01$ & $3.00 E+00$ \\
$9.44 E+00$ & $6.33 E-01$ & $4.16 E+00$ & $6.53 E-01$ & $5.69 E-01$ & $3.20 E+00$ \\
$1.60 E+01$ & $7.17 E-01$ & $4.69 E+00$ & $1.11 E+00$ & $9.64 E-01$ & $5.42 E+00$ \\
$250 E+01$ & $7.82 E-01$ & $5.09 E+00$ & $1.73 E+00$ & $1.51 E+00$ & $8.47 E+00$ \\
$3.18 E+01$ & $8.14 E-01$ & $5.29 E+00$ & $2.20 E+00$ & $1.92 E+00$ & $1.08 E+01$ \\
$3.65 E+01$ & $8.32 E-01$ & $5.40 E+00$ & $253 E+00$ & $2.20 E+00$ & $1.24 E+01$ \\
\hline
\end{tabular}


Table D.4: Partial Pressure and Loading data for the H2S-MEA system at $49^{\circ} \mathrm{C}$

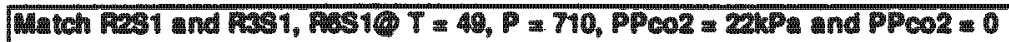

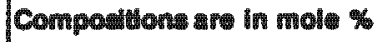

\begin{tabular}{|c|c|c|c|c|c|c|c|c|c|}
\hline \multirow[b]{2}{*}{$\mathrm{pP}$} & \multicolumn{4}{|c|}{ With PP CO2 kept 22kP } & \multicolumn{5}{|c|}{ No CO2 in system } \\
\hline & stor & Iph H2S & H2S & $y \cdot R 2, R 3$ & Apha $\mathrm{H}$ & H2S & $y+R_{2}, R_{3}$ & $y+76$ & \\
\hline 0.01 & 0.533 & $6.45 E-05$ & 0.0004 & 0.001 & 0.020 & 0.13 & 0.001 & & 0.0014 \\
\hline 0.02 & 0.533 & $1.03 E-04$ & 0.0007 & 0.002 & 0.025 & 0.17 & 0.002 & & 0.0023 \\
\hline 0.03 & 0.533 & 206EA & 0.0014 & 0.005 & 0.037 & 0.25 & 0.005 & & 0.0046 \\
\hline 0.05 & 0.533 & $3.43 E-O 4$ & 0.002 & 0.008 & 0.049 & 0.34 & 0.008 & & 0.0076 \\
\hline 0.06 & 0.533 & 4.10E-OA & 0.003 & 0.000 & 0.054 & 0.37 & 0.009 & & 0.0091 \\
\hline 0.13 & 0.532 & 8.19E-OA & 0.005 & 0.018 & 0.078 & 0.53 & 0.018 & & 0.018 \\
\hline 0.57 & 0.531 & $3.68 \mathrm{E}-03$ & 0.024 & 0.080 & 0.168 & 1.14 & 0.08 & & 0.08 \\
\hline 1.00 & 0.529 & $6.42 \mathrm{E}-03$ & 0.04 & 0.14 & 0.220 & 1.48 & 0.14 & & 0.14 \\
\hline 1.27 & 0.529 & 0.01 & 0.05 & 0.18 & 0.277 & 1.87 & 0.18 & & 0.18 \\
\hline 8.44 & 0.505 & 0.05 & 0.34 & 1.19 & 0.511 & 3.39 & 1.19 & & 1.21 \\
\hline 15.61 & 0.483 & 0.09 & 0.61 & 2.20 & 0.612 & 4.03 & 2.20 & & 2.23 \\
\hline $22 \pi$ & 0.463 & 0.13 & 0.86 & 3.21 & 0.674 & 4.42 & 3.21 & & 3.25 \\
\hline 29.94 & 0.444 & 0.17 & 1.10 & 4.22 & 0.717 & 4.69 & 4.22 & & 4.28 \\
\hline 37.11 & 0.427 & 0.20 & 1.32 & 5.23 & 0.750 & 4.89 & 5.23 & & 5.30 \\
\hline 4.28 & 0.410 & 0.23 & 1.53 & 8.24 & 0.776 & 5.05 & 6.24 & & 6.33 \\
\hline 51.4 & 0.395 & 0.26 & 1.72 & 7.25 & 0.797 & 5.19 & 7.25 & & 7.35 \\
\hline 58.61 & 0.380 & 0.29 & 1.91 & 8.28 & 0.815 & 5.20 & 8.26 & & 8.37 \\
\hline 65.78 & 0.386 & 0.32 & 208 & 9.28 & 0.830 & 5.39 & 9.20 & & 9.40 \\
\hline 7295 & 0.353 & 0.34 & 2.24 & 10.27 & 0.843 & 5.47 & 10.27 & & 10.42 \\
\hline 80.12 & 0.341 & 0.37 & 240 & 11.28 & 0.854 & 5.54 & 11.28 & & 11.45 \\
\hline
\end{tabular}


Table D.5: Partial Pressure and Loading data for the H2S-MEA system

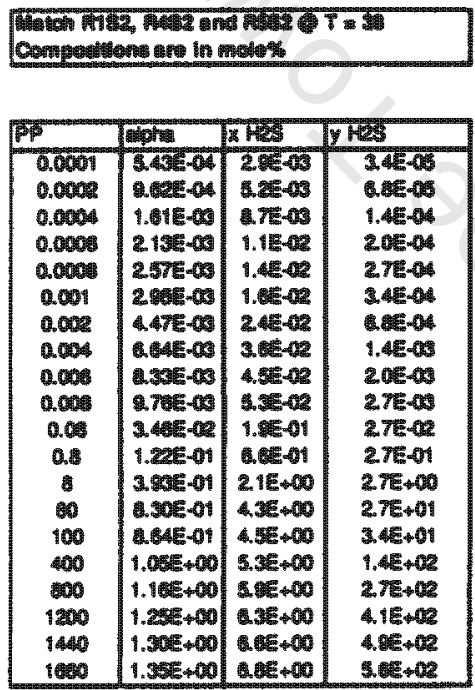

\begin{tabular}{|c|c|c|c|c|}
\hline \multicolumn{5}{|c|}{ 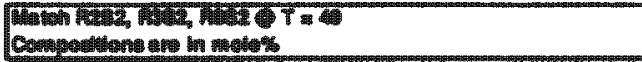 } \\
\hline emphe & & Ths & T) & Yमma \\
\hline (3.3) & (c) & $6.67 \%$ & Thes & D. 15 \\
\hline 1.71) & 需 & atet & 1.14 & 1. \\
\hline $1.00 \mathrm{cos}$ & 1,000 & 2.011 & 1.4 (1) & 1.410 .4 \\
\hline 3.20 & $300 \mathrm{eds}$ & 0.02 & 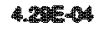 & 4.28 \\
\hline 3 Jetos & coneds & 00 & 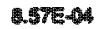 & 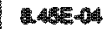 \\
\hline 0.01 & 0.01 & 0.0 & 1.ASEA & 1.41: \\
\hline 0.0 & 0.05 & a.to & $7.14 E-10$ & $7.04 E-4 B$ \\
\hline 0.10 & 0.00 & 0.51 & 43 & 0.13 \\
\hline 0.10 & 1.02 & 25 & a. 14 & 0.14 \\
\hline 0.145 & 2 & 0.67 & 021 & 02 \\
\hline 0.198 & 3 & 0.97 & 0 & 0.42 \\
\hline 03 & 10 & 1.77 & 1.48 & 1.44 \\
\hline 1. & a & 240 & 2 25e & 22 \\
\hline $\cos$ & 0 & 281 & 4.20 & 4.23 \\
\hline a.t & 50 & 3 & 7.14 & 7.04 \\
\hline 0.76 & 100 & 40 & 14.28 & 14.06 \\
\hline 0.9 & 200 & 40 & 2) & 217 \\
\hline a.te & 300 & 4.6 & 426 & 4.2.5 \\
\hline 12 & 4 & 50 & 57.1. & 59u \\
\hline 1.09 & 500 & S.2. & 71.48 & 70 \\
\hline 1.06 & 600 & 5.45 & . 71 & 45? \\
\hline 1.0 & 700 & 3.52 & 100,00 & ats \\
\hline 1.0 & 70 & 5.54 & 101.43 & 100.00 \\
\hline
\end{tabular}


Sample calculation for the conversion of gas loading $(\alpha)$ to liquid composition $x$

Loading:

0.02 mole $_{2} \mathrm{~S}$ per mole MEA

0 mole $\mathrm{CO}_{2}$ per mole MEA

Thus the total number of moles based on 1 mole of MEA is 14.6 according to the following contributions:

$\begin{aligned} \mathrm{H}_{2} \mathrm{~S} & =0.02 \\ \text { MEA } & =1 \\ \text { water } & =\text { moles MEA } \times \frac{\mathrm{MMr}_{\mathrm{MEA}}}{\mathrm{MMr}_{\mathrm{H}_{2} \mathrm{O}}} \times \frac{\mathrm{wt} \% \mathrm{H}_{2} \mathrm{O}}{\text { wt } \% \mathrm{MEA}} \\ & =1 \times \frac{61}{18} \times \frac{80}{20} \\ & =13.55\end{aligned}$

The mole fraction of $\mathrm{H}_{2} \mathrm{~S}$ in the liquid is given by:

$$
\begin{aligned}
x_{\mathrm{H}_{2} \mathrm{~S}} & =\frac{m_{\mathrm{H}_{3} \mathrm{~S}}}{m_{\mathrm{H}_{2} \mathrm{~S}}+\mathrm{MEA}+m_{\mathrm{H}_{2} \mathrm{O}}+m_{\mathrm{CO}_{3}}} \\
& =\frac{0.02}{0.02+1+13.55+0} \\
& =1.3727 \times 10^{-3}
\end{aligned}
$$

The molalities given in EquationD.0.1include both physically dissolved and chemical bonded hydrogen sulphide, alkanoamine, water and carbon dioxide.

The calculation is similar for the DEA with the appropriate molecular weight and weight percentages. 


\section{Appendix E}

\section{Additional Data on the MTA method}

Table E.1: Recalculating solutions of Hallale(1998) using the MTA method **N-MINLP refers to Equation2.10 and N-Hallale to Equation2.9

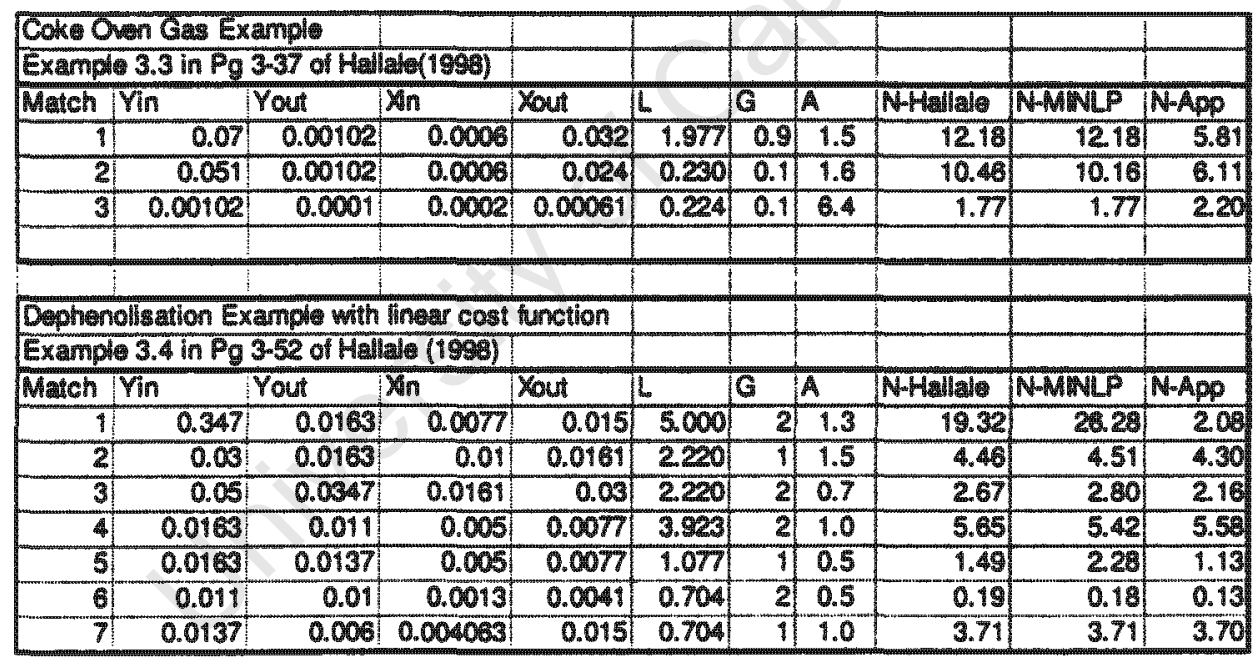




\begin{tabular}{|c|c|c|c|c|c|c|c|c|c|c|}
\hline \multicolumn{11}{|c|}{ Ammonity removel ample } \\
\hline \multicolumn{11}{|c|}{ Example $4.1 \mathrm{in} \mathrm{Pg} 4-16$ of Hallale (1998) } \\
\hline Match & Vin & Yout & Xn & Xout & 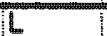 & C & A & NHHallas & NHMNLP & $N=A \infty O$ \\
\hline 1 & 0.0107 & 0.005 & 0.009 & 0.017 & 2480 & 3.5 & 1.42 & 4.22 & 6.25 & 4.22 \\
\hline 2 & 0.009 & 0.005 & 0.0038 & 0.0071 & 1.800 & 7.5 & 1.00 & 9.00 & $3.92 E+14$ & 8.70 \\
\hline 3 & 0.006 & 0.005 & 0.0045 & 0.006 & 1.000 & 0.5 & 2.00 & 200 & 200 & 2.40 \\
\hline 4 & 0.01 & 0.009 & 0.0061 & 0.0075 & 1.000 & 1.5 & 0.57 & 0.45 & 0.45 & 0.30 \\
\hline (5) & 0.011 & 0.0107 & 0.0075 & 0.0085 & 1.000 & 3.5 & 0.29 & 0.21 & 0.20 & 0.11 \\
\hline (6) & 0.005 & 0.0032 & 0.0017 & 0.0038 & 1.800 & 2 & 0.75 & 253 & 3.37 & 22 \\
\hline 7 & 0.005 & 0.003 & 0.0025 & 0.0045 & 1.000 & 1 & 1.02 & 3.81 & 0.00 & 4.00 \\
\hline (8) & 0.005 & 0.0025 & 0.003332 & 0.000 & 1.760 & 4 & 0.69 & 4.13 & 4.13 & 3.22 \\
\hline 9 & 0.005 & 0.0025 & 0 & 0.009 & 0.700 & 25 & 0.56 & 274 & 274 & 1.67 \\
\hline 10 & 0.005 & 0.0025 & 0.002252 & 0.00338 & 1.7801 & 0.5 & 7.12 & 0.53 & 0.53 & 1.12 \\
\hline Tा & 0.003 & 0.0025 & 0.002416 & 0.00343 & T.780! & 3.6 & 0.99 & 0.39 & 0.39 & 0.39 \\
\hline 12 & (032) & 0.001 & 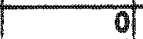 & 0.00242 & 1.780 & 2 & 1.78 & 1.17 & 1.20 & 1.47 \\
\hline & & & & & & & & & & \\
\hline \multicolumn{5}{|c|}{ Dephenolisation ax ample mploying mixim settiers } & & & & & & \\
\hline \multicolumn{11}{|c|}{ Exampd 4.2 in Po 4.29 of Haldale (1998). Also in Po $5-6$ of Hallale (1908) } \\
\hline Match & Yin & Yout & Wh & Xout & $\bar{L}$ & G & $\bar{A}$ & 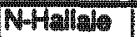 & NWMNLP & NApp \\
\hline 1 & 0.0347 & 0.0163 & 0.0077 & 0.015 & 5 & 2) & 1.25 & 7.29 & 7.41 & 6.57 \\
\hline 2 & 0.09 & 0163 & 0.01 & 0.0161 & 222 & 1 & 1.45 & 4.48 & 4.51 & 4.30 \\
\hline 3 & 0.05 & 334 & 0.01611 & 0.03 & 222 & 2. & 0.73 & 267 & 280 & 2.16 \\
\hline 4 & 0163 & 0.0126 & 0.005 & 0.0077 & 296 & 2 & 0.74 & 230 & 3.52 & 2.11 \\
\hline 5 & ग16्ञा & 0.0109 & 0.005 & 0.0077 & 2 & 1 & 1.00 & 0.00 & 0.00 & 6.00 \\
\hline (8) & 109 & 0.008 & 0.0013 & 0.0087 & 0.704 & 1 & 0.99 & 1.21 & 5.56 & 1.23 \\
\hline 7 & 0.0123 & 0.011 & 0.000460 & 0.015 & 0.704 & $\pi$ & 0.50 & 217 & 217 & 8.20 \\
\hline
\end{tabular}

\begin{tabular}{|c|c|c|c|c|c|c|c|c|c|c|}
\hline \multicolumn{11}{|c|}{ 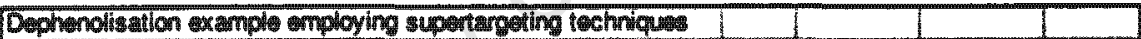 } \\
\hline \multicolumn{11}{|c|}{ Examplo 5.1 in $\mathrm{Pg} 5.5$ of Hallate (1000) । I I } \\
\hline \multicolumn{2}{|c|}{ Match ivn } & \multirow{2}{*}{$\frac{\text { You }}{0.0156}$} & den & rout & $1=$ & \multirow{2}{*}{ Q } & $A$ & \multirow{2}{*}{ N4hinglin } & MWINLS & NAPP \\
\hline 1 & 0.0339 & & 0.0077 & 0.015 & 5.000 & & $\sqrt[2]{1.25}$ & & 13.31 & 9.98 \\
\hline 2 & 0.03 & 0.0856 & 0.01 & 0.0161 & 2330 & & $1 \longdiv { 1 . 5 2 }$ & 6.80 & 6.88 & 5.06 \\
\hline 3 & 0.05 & 0.0090 & 0.0161 & 0.03 & 2330 & & $2 \longdiv { 0 . 7 6 }$ & 2.8 & 290 & 2.41 \\
\hline 4 & 0.0156 & 0.0107 & 0.005 & 0.0077 & 3.509! & & $2 \longdiv { 0 . 0 0 }$ & 14.70 & 11.68 & 10.89 \\
\hline 5 & 0.0156 & 0.0118 & 0.005 & 0.0077 & 1.407 & & 0.70 & 6.2 & 6.25 & 3.80 \\
\hline 6 & 107 & .011 & 0.0013 & 0.00426 & 0.540 & & 20.30 & 0.16 & 0.20 & 0.08 \\
\hline & 0.01181 & 0.0061 & 0.00425 & 0.015 & 0.540 & & 0.76 & 9.4 & 9.42 & 5.46 \\
\hline & & & & & & & & & & \\
\hline \multirow{2}{*}{\multicolumn{11}{|c|}{ 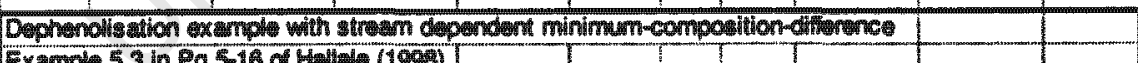 }} \\
\hline \multirow{2}{*}{ 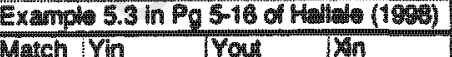 } & & & & & & & & & & \\
\hline & & Yout & Xn & Xout & I & 19 & A & 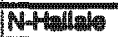 & NWMUNPP & NoApp \\
\hline is & 0.0339 & 0.01545 & 0.0076 & 0.015 & 5.00 & 2 & 21.25 & 1236 & 1231 & 8.89 \\
\hline \multirow{2}{*}{$\begin{array}{r}2 \\
3\end{array}$} & 0.03 & 0.01545 & 0.01 & 0.0122 & 233 & & 11 1.52 & 8.41 & 8.44 & 5.43 \\
\hline & 05 & 0.0339 & 0.0161 & 0.03 & 2.33 & & 20.76 & 68 & 290 & 241 \\
\hline & 0.0154 & 0.0107 & 0.005 & 0.0076 & 3.65 & & 20.81 & 1.16 & 13.65 & 10.44 \\
\hline \multirow[t]{2}{*}{5} & 0.0154 & 0.0119 & 0.005 & 0.0076 & 1.35 & 1 & \begin{tabular}{|l|l}
0.67 \\
\end{tabular} & 5.68 & 5.68 & 3.33 \\
\hline & 0.0107 & & 0.0013 & 0.0038 & 0.54 & & 0.38 & & 0.16 & 0.09 \\
\hline \multirow[t]{2}{*}{7} & 0.0110 & 0.000 & 0.000805 & 0.015 & 0.54 & & 10.76 & 8.00 & 8.11 & 9.63 \\
\hline & & & & & & & & & & \\
\hline \multicolumn{11}{|c|}{ 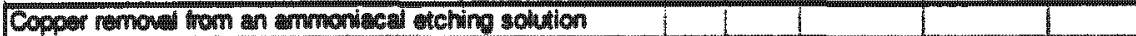 } \\
\hline \multicolumn{4}{|c|}{ Example 5.4 in Pg $5-20$ of Hinlahe (1020) } & & & & & & & \\
\hline \multicolumn{2}{|c|}{ Match Yin } & Yout & Xin & Wout & IL & C & A & 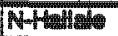 & WMMUN & NAPP \\
\hline 11 & 0.06 & 0.0235 & 0.08 & 0.043 & 0.279 & 0.1 & 13.80 & 3.09 & 3.03 & 261 \\
\hline 2 & 0.13 & 0.1 & 0.043 & 0.07 & 0.279 & 0.3 & 1.52 & 0.34 & 0.34 & 0.41 \\
\hline 3 & 0.0235 & 0.02 & 0.001 & 0.02 & 0.019 & 0.1 & 111.28 & 0.43 & 0.42 & 0.48 \\
\hline
\end{tabular}




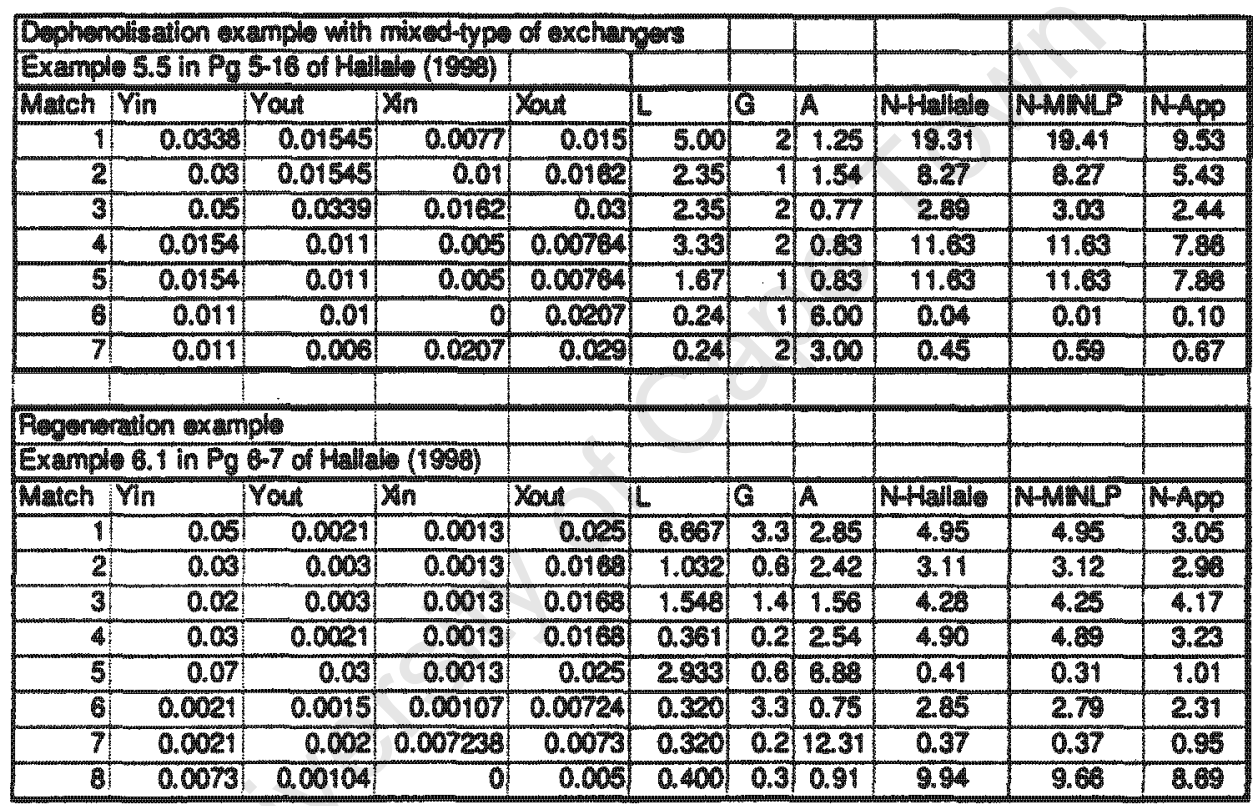

